\title{
ASYMMETRIC SYNTHESIS OF (-)-DIHYDROXANTHATIN BY THE STEREOSELECTIVE OSHIMA REACTION.
}

\author{
MICAHEL A. EVANS AND JAMES P. MORKEN* \\ DEPARTMENT OF CHEMISTRY, VENABLE AND KENAN LABORATORIES, THE \\ UNIVERSITY OF NORTH CAROLINA AT CHAPEL HILL, CHAPEL HILL, NORTH \\ CAROLINA, 27599-3290.
}

General. Infrared (IR) spectra were recorded on an Applied Systems React-IR 1000 (Model 0011002), $v_{\max }$ in $\mathrm{cm}^{-1}$. Proton NMR spectra were recorded on Bruker model DRX $500\left({ }^{1} \mathrm{H} 500 \mathrm{MHz}\right)$, and Bruker model DRX $400\left({ }^{1} \mathrm{H} 400 \mathrm{MHz}\right)$ spectrometers. Chemical shifts are reported in ppm from tetramethylsilane with the solvent resonance as the internal standard $\left(\mathrm{CDCl}_{3}: \delta=7.24 \mathrm{ppm}\right.$ for $\left.{ }^{1} \mathrm{H}\right)$. Data are reported as follows: chemical shift, multiplicity $(\mathrm{s}=$ singlet, $\mathrm{d}=$ doublet, $\mathrm{t}=$ triplet, $\mathrm{q}=$ quartet, $\mathrm{qu}=$ quintet, $\mathrm{sx}=$ sextet, $\mathrm{b}=$ broad, $\mathrm{m}=$ multiplet $)$, coupling constants $(\mathrm{Hz})$, integration and assignment. Carbon NMR were recorded on Bruker model DRX $500\left({ }^{13} \mathrm{C} 125 \mathrm{MHz}\right)$ and Bruker model DRX $400\left({ }^{13} \mathrm{C} 100 \mathrm{MHz}\right)$ spectrometers with complete proton decoupling. Chemical shifts are reported in ppm from tetramethylsilane with the solvent as the internal standard $\left(\mathrm{CDCl}_{3}: \delta=77.0\right.$ $\mathrm{ppm}$ ). High-resolution mass spectrometry were performed by the University of Minnesota department of chemistry mass spectrometry laboratory with a Finnigan MAT95 mass spectrometer. Lowresolution mass spectrometry was performed using a Micromass Quattro II triple quadrupole mass spectrometer. Optical rotations were determined using a Jasco DIP-1000 polarimeter and are reported as follows: $[\alpha]_{\mathrm{D}}$, concentration $(c=\mathrm{g} / 100 \mathrm{~mL})$, solvent.

Liquid chromatography was performed using forced flow (flash chromatography) of the indicated solvent system on Scientific Absorbents or Sigma silica gel $60\left(\mathrm{SiO}_{2}, 230-400\right.$ mesh). Thin layer chromatography was preformed on EM science $0.25 \mathrm{~mm}$ silica gel 60 plates. Visualization was achieved with phosphomolybdic acid in ethanol, potassium permanganate in water, and cerium ammonium molybdate in water, each followed by heating.

Tetrahydrofuran was distilled from sodium/benzophenone ketyl under a nitrogen atmosphere. Acetonitrile and acetone were purchased from Fisher Scientific and were used with no further purification. Dichloromethane was dried by being passed through a column of neutral alumina under $\mathrm{N}_{2}$ immediately prior to use. All metals and butyl vinyl ether were purchased from Aldrich chemical company. 


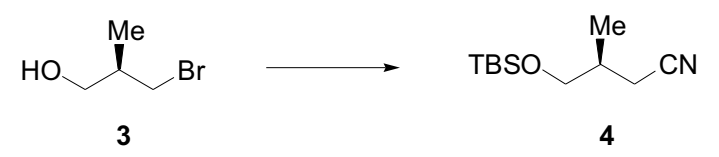

(S)-4-(tert-butyldimethylsilyloxy)-3-methylbutanenitrile (4). Commercially available $(R)$-3bromo-2-methyl-propanol $(13.12 \mathrm{~g}, 85.78 \mathrm{mmol})$ was added to a $250 \mathrm{ml}$ 3-neck RBF under argon. $137 \mathrm{ml}$ DMSO was added, followed by $4.62 \mathrm{~g}(94.4 \mathrm{mmol})$ of sodium cyanide. The reaction stirred a total of 28 hours, during which time all cyanide was dissolved. The solution was poured out into water, and was extracted three times with EtOAc. To extract the remainder of the compound, the aqueous solution was carefully acidified to $\mathrm{pH}=5$ using $10 \%$ sulfuric acid. After extracting the aqueous layer three more times, all organic layers were combined and were washed with brine. The solution was dried over $\mathrm{MgSO}_{4}$ before removing the solvent in-vacuo. $7.59 \mathrm{~g}(76.5 \mathrm{mmol}, 89 \%)$ of the crude hydroxynitrile was collected and used directly in the next step.

The crude hydroxynitrile was added to a $250 \mathrm{ml} \mathrm{RBF}$ before being dissolved in $77 \mathrm{ml} \mathrm{CH}_{2} \mathrm{Cl}_{2}$. $17.3 \mathrm{~g}(115 \mathrm{mmol})$ tert-butyldimethylsilyl chloride and $2.80 \mathrm{~g}(22.9 \mathrm{mmol})$ 4-dimethylaminopyridine was then added. The solution was cooled to $0{ }^{\circ} \mathrm{C}$ before adding $21.3 \mathrm{ml}(153 \mathrm{mmol})$ triethylamine. After stirring 30 minutes, the reaction was warmed to room temperature. After 3 hours at this temperature, a significant amount of white triethylamine hydrochloride salt had precipitated out of solution. To work up the reaction, it was first poured into a $10 \%$ citric acid solution, and was extracted with ethyl acetate three times. The organic layer was washed once more with $10 \%$ citric acid, then with sodium bicarbonate, and finally with brine before drying over $\mathrm{MgSO}_{4}$. After removing the solvent in vacuo, the material was purified using silica chromatography. (15:1 hexanes:EtOAc) A total of $14.00 \mathrm{~g}(65.63 \mathrm{mmol}, 77 \%$ over 2 steps $)$ of the desired silyl ether 4 was isolated. $\mathrm{R}_{f}=0.56\left(6: 1\right.$ Hexanes:EtOAc); ${ }^{1} \mathrm{H}$ NMR $\left(\mathrm{CDCl}_{3}, 400 \mathrm{MHz}\right) \delta 3.57(\mathrm{dd}, J=10.1 \mathrm{~Hz}, 4.6$ $\mathrm{Hz}, 1 \mathrm{H}), 3.38$ (dd, $J=10.1 \mathrm{~Hz}, 7.4 \mathrm{~Hz}, 1 \mathrm{H}), 2.44(\mathrm{dd}, J=16.7 \mathrm{~Hz}, 5.5 \mathrm{~Hz}, 1 \mathrm{H}), 2.30$ (dd, $J=16.7$ $\mathrm{Hz}, 7.1 \mathrm{~Hz}, 1 \mathrm{H}), 1.94-2.05(\mathrm{~m}, 1 \mathrm{H}), 1.02(\mathrm{~d}, J=6.8 \mathrm{~Hz}, 3 \mathrm{H}), 0.87(\mathrm{~s}, 9 \mathrm{H}), 0.03(\mathrm{~s}, 6 \mathrm{H}) ;{ }^{13} \mathrm{C}$ NMR $\left(\mathrm{CDCl}_{3}, 100 \mathrm{MHz}\right) \delta 118.9,66.0,33.2,25.8,20.9,18.2,15.8,-5.5,-5.6$; IR (film) $v_{\max } 2958,2931$, 2887, 2680, 2248, 1472, 1254, 1102, 840, 778; LRMS (ESI+) calc'd. for $\mathrm{C}_{11} \mathrm{H}_{23} \mathrm{NOSi}[\mathrm{M}+\mathrm{Na}]^{+}$ 236.1, found: 236.0 .

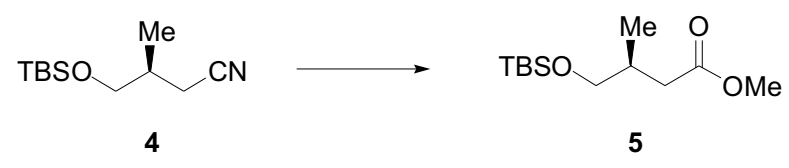

(S)-Methyl 4-(tert-butyldimethylsilyloxy)-3-methylbutanoate (5). $14.40 \mathrm{~g} \mathrm{(67.48} \mathrm{mmol)} \mathrm{of} \mathrm{nitrile}$ 4 was added to a flame-dried $11 \mathrm{RBF}$. The oil was then dissolved in $337 \mathrm{ml} \mathrm{CH}_{2} \mathrm{Cl}_{2}$ before cooling the flask to $-78{ }^{\circ} \mathrm{C}$. $80.98 \mathrm{ml}(80.98 \mathrm{mmol})$ of a $1 \mathrm{M}$ solution of DIBAL-H in hexanes was then slowly added over a period of 10 minutes. After an additional 10 minutes of stirring, the reaction was allowed to warm to room temperature and stir for 1.5 hours. It was then poured out into a 21 Erlenmeyer. The reaction was then quenched with saturated ammonium chloride. After a 2 minute induction period, the quench began generating heat and was therefore cooled with an ice bath. After reaction ceased, 5\% sulfuric acid was slowly added with steady swirling until the opaque slurry became two distinct layers. With the $\mathrm{pH}$ at 2-3, the organic layer was separated, and the aqueous layer was extracted three times with $\mathrm{CH}_{2} \mathrm{Cl}_{2}$. The organic layers were combined, neutralized with saturated sodium bicarbonate, and were dried with a brine wash. The solution was further dried over $\mathrm{MgSO}_{4}$ before removing the solvent in-vacuo. $14.02 \mathrm{~g}$ (64.79 $\mathrm{mmol}, 96 \%$ yield $)$ of the desired 
aldehyde was obtained as a clear iridescent yellow oil. The material was used without further purification.

$14.02 \mathrm{~g}$ (64.79 mmol) of aldehyde was transferred into a 1L RBF, and was dissolved in $234 \mathrm{ml} 2$ methyl-2-propanol. Next, $103 \mathrm{ml}(972 \mathrm{mmol})$ 2-methyl-2-butene was added, and the reaction was cooled to $0{ }^{\circ} \mathrm{C}$. A solution of $13.47 \mathrm{~g}(149.0 \mathrm{mmol})$ sodium chlorite and $17.88 \mathrm{~g}(129.5 \mathrm{mmol})$ sodium dihydrogenphosphate monohydrate in $86 \mathrm{ml}$ water was then slowly cannulated into the reaction vessel. The reaction and the sodium chlorite solution were kept from any light source during this process. The reaction was allowed to warm to room temperature, and the resulting yellow solution became clear over the course of 2 hours. During this time, a large amount of white precipitate also formed. The solution was poured into $500 \mathrm{ml}$ of water before being extracted three times with EtOAc. The organic layers were combined, washed with brine twice, and were dried over $\mathrm{MgSO}_{4}$ before removing the solvent in-vacuo. The oil was then taken up in a solution of hexanes and

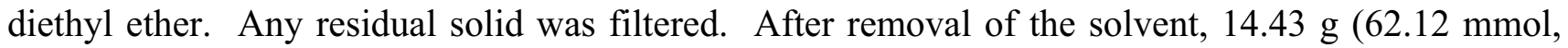
$96 \%$ yield) of the desired acid was obtained as a slightly opaque yellow oil. This material used without further purification.

$14.43 \mathrm{~g}(62.12 \mathrm{mmol})$ of crude acid was dissolved in $126 \mathrm{ml}$ benzene and $31 \mathrm{ml}$ methanol in a $500 \mathrm{ml} \mathrm{RBF}$. The solution was cooled to $0{ }^{\circ} \mathrm{C}$ before $31.68 \mathrm{ml}(63.37 \mathrm{mmol})$ of a $2 \mathrm{M}$ solution of trimethylsilyldiazomethane in ether was slowly added via addition funnel over the period of 15 minutes. The reaction was run in the dark, and the evolution of $\mathrm{N}_{2}$ was noted immediately. The reaction was allowed to warm to room temperature, and stirred for 30 minutes before the solvent was removed in vacuo. The crude oil was purified by silica chromatography (30:1 hexanes:EtOAc),

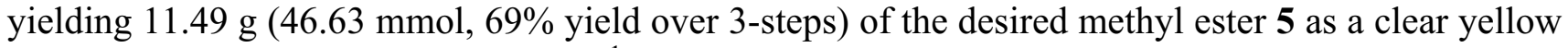
oil. $\mathrm{R}_{f}=0.34\left(20: 1\right.$ Hexanes:EtOAc); ${ }^{1} \mathrm{H}$ NMR $\left(\mathrm{CDCl}_{3}, 400 \mathrm{MHz}\right) \delta 3.65(\mathrm{~s}, 3 \mathrm{H}), 3.48(\mathrm{dd}, J=9.8$ $\mathrm{Hz}, 5.2 \mathrm{~Hz}, 1 \mathrm{H}), 3.38$ (dd, $J=9.8 \mathrm{~Hz}, 6.4 \mathrm{~Hz}, 1 \mathrm{H}), 2.47$ (dd, $J=17.2 \mathrm{~Hz}, 7.8 \mathrm{~Hz}, 1 \mathrm{H}), 2.03-2.18$ (m, $2 \mathrm{H}), 0.91(\mathrm{~d}, 6.6 \mathrm{~Hz}, 3 \mathrm{H}), 0.865(\mathrm{~s}, 9 \mathrm{H}), 0.01(\mathrm{~s}, 6 \mathrm{H}) ;{ }^{13} \mathrm{C} \mathrm{NMR}\left(\mathrm{CDCl}_{3}, 100 \mathrm{MHz}\right) \delta 173.6,67.4$, 51.3, 37.9, 33.0, 25.9, 18.3, 16.5, -5.5; IR (film) $v_{\max }$ 2956, 2931, 2858, 1742, 1258, 1096, 837; LRMS (ESI+) calc'd. for $\mathrm{C}_{12} \mathrm{H}_{26} \mathrm{O}_{3} \mathrm{Si}[\mathrm{M}+\mathrm{Na}]^{+}$269.2, found: 269.1.

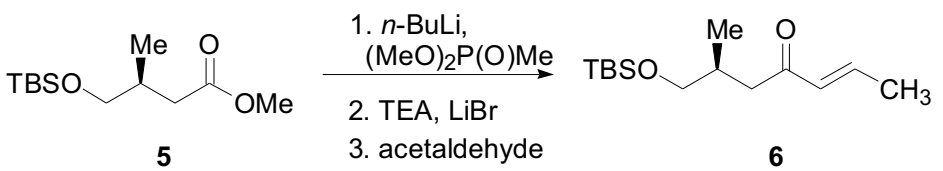

(S,E)-7-(tert-Butyldimethylsilyloxy)-6-methylhept-2-en-4-one (6). A dry $500 \mathrm{ml}$ RBF was charged with $13.3 \mathrm{ml}(122 \mathrm{mmol})$ dimethyl methylphosphonate and $144 \mathrm{ml} \mathrm{THF}$. The solution was cooled to $-78{ }^{\circ} \mathrm{C}$, and $50.3 \mathrm{ml}(122 \mathrm{mmol})$ of a $2.43 \mathrm{M}$ solution of $n$-BuLi in hexanes was slowly added. After $40 \mathrm{~min}, 10.04 \mathrm{~g} \mathrm{(40} \mathrm{mmol)} \mathrm{ester} \mathrm{3a} \mathrm{in} 60 \mathrm{ml}$ of THF was transferred via canula into the resultant slurry. After stirring an additional hour, the reaction was poured into saturated aqueous ammonium chloride and was extracted with EtOAc. The organic layers were combined and washed with brine and dried over $\mathrm{MgSO}_{4} .14 .6290 \mathrm{~g}$ of the crude product was isolated after removing the solvent in vacuo. The crude material was transferred to a dry $500 \mathrm{ml} \mathrm{RBF} \mathrm{(} \sim 40 \mathrm{mmol}$ assumed and also added were $161 \mathrm{ml}$ of THF, and $5.650 \mathrm{~g}(65.0 \mathrm{mmol})$ of lithium bromide respectively. The vessel was cooled to $0{ }^{\circ} \mathrm{C}$, and $7.25 \mathrm{ml}(52.0 \mathrm{mmol})$ of triethyl amine was slowly added. After stirring for 40 $\min , 7.3 \mathrm{ml}(130 \mathrm{mmol})$ of acetaldehyde was added to the resultant slurry, and the reaction was warmed to room temperature. After stirring for $2 \mathrm{~h}$, another $7.3 \mathrm{ml}(130 \mathrm{mmol})$ of acetaldehyde was added, and the reaction was stirred an additional $2 \mathrm{~h}$. It was then quenched by pouring out into an aquous solution of $10 \%$ citric acid. The product was extracted with ether which was then washed with brine and dried over $\mathrm{MgSO}_{4}$. The solvent was removed in vacuo and the crude oil was purified via flash silica chromatography. (10:1 hexanes:EtOAc) $7.7989 \mathrm{~g}$ (30.4098 mmol, 75\% over two 
steps) of the desired ketone 6 was isolated as a clear faint yellow oil. $\mathrm{R}_{f}=0.44$ (7.5:1 Hexanes:EtOAc); ${ }^{1} \mathrm{H}$ NMR $\left(\mathrm{CDCl}_{3}, 400 \mathrm{MHz}\right) \delta 6.83(\mathrm{dq}, J=15.7 \mathrm{~Hz}, 6.8 \mathrm{~Hz}, 1 \mathrm{H}), 6.10(\mathrm{dq}, J=$ 15.7, 1.7, 1H), $3.46(\mathrm{dd}, J=9.8 \mathrm{~Hz}, 5.2 \mathrm{~Hz}, 1 \mathrm{H}), 3.37(\mathrm{dd}, J=9.8 \mathrm{~Hz}, 6.3 \mathrm{~Hz}, 1 \mathrm{H}), 2.71(\mathrm{dd}, J=14.9$ $\mathrm{Hz}, 5.0 \mathrm{~Hz}, 1 \mathrm{H}), 2.11-2.26(\mathrm{~m}, 2 \mathrm{H}), 1.87(\mathrm{dd}, J=6.8 \mathrm{~Hz}, 1.7 \mathrm{~Hz}, 3 \mathrm{H}), 0.85-0.90(\mathrm{~m}, 12 \mathrm{H}), 0.012(\mathrm{~s}$, $3 \mathrm{H}), 0.006(\mathrm{~s}, 3 \mathrm{H}) ;{ }^{13} \mathrm{C} \mathrm{NMR}\left(\mathrm{CDCl}_{3}, 100 \mathrm{MHz}\right) \delta 200.1,142.1,132.4,67.5,43.6,32.5,25.8,18.2$, 18.1, 16.7, -5.49, -5.52; IR (film) $v_{\max }$ 2956, 2931, 2885, 2858, 1713, 1254, 1096, 837; LRMS (ESI+) calcd. for $\mathrm{C}_{14} \mathrm{H}_{28} \mathrm{O}_{2} \mathrm{Si}[\mathrm{M}+\mathrm{Na}]^{+} 257.2$, found: 257.1 .

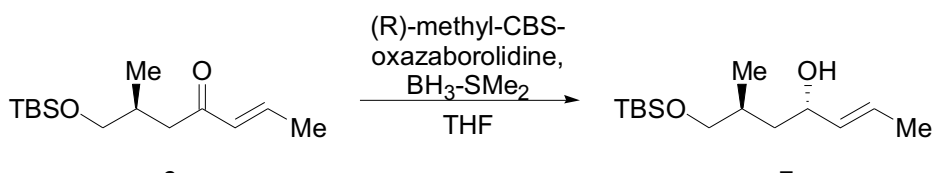

(4S,6S,E)-7-(tert-butyldimethylsilyloxy)-6-methylhept-2-en-4-ol (7). A flame-dried $250 \mathrm{ml}$ RBF was charged with $4.1754 \mathrm{~g}(16.281 \mathrm{mmol})$ ketone $\mathbf{3 b}$ and $81 \mathrm{ml}$ THF. The vessel was cooled to -20 ${ }^{\circ} \mathrm{C}$ and $1.63 \mathrm{ml}(1.63 \mathrm{mmol})$ of $1.00 \mathrm{M}(\mathrm{R})$-methyl-CBS-oxazaborolidine in toluene was added. Finally, $10.29 \mathrm{ml}(108.5 \mathrm{mmol})$ of borane-methyl sulfide complex was slowly added over $10 \mathrm{~min}$. The reaction stirred for $50 \mathrm{~min}$ at this temperature before being slowly quenched with $40 \mathrm{ml}$ of methanol over the period of about an hour. The reaction was warmed to room temperature, and after the majority of gas evolution subsided, the solvent was removed in vacuo. The crude oil was purified by flash silica chromatography (12:1 hexanes:EtOAc) yielding $3.47 \mathrm{~g}(13.4 \mathrm{mmol}, 83 \%)$ as a clear colorless oil with $\mathrm{dr}=10.5: 1 . \mathrm{R}_{f}=0.49$ (4:1 Hexanes:EtOAc); ${ }^{1} \mathrm{H}$ NMR $\left(\mathrm{CDCl}_{3}, 400 \mathrm{MHz}\right) \delta 5.65$ $(\mathrm{dq}, J=15.3 \mathrm{~Hz}, 6.4 \mathrm{~Hz}, 1 \mathrm{H}), 5.48(\mathrm{dd}, J=15.3 \mathrm{~Hz}, 1.5 \mathrm{~Hz}, 1 \mathrm{H}), 4.05-4.14(\mathrm{~m}, 1 \mathrm{H}), 3.49$ (dd, $J=9.9$ $\mathrm{Hz}, 5.0 \mathrm{~Hz}, 1 \mathrm{H}), 3.39$ (dd, $J=9.9 \mathrm{~Hz}, 7.4 \mathrm{~Hz}, 1 \mathrm{H}), 2.95$ (bs, 1H), 1.72-1.83 (m, 1H), 1.67 (d, $J=6.4$ $\mathrm{Hz}, 3 \mathrm{H}), 1.51$ (ddd, $J=14.2 \mathrm{~Hz}, 9.1 \mathrm{~Hz}, 7.0 \mathrm{~Hz}, 1 \mathrm{H}), 1.40$ (ddd, $J=14.2 \mathrm{~Hz}, 5.8 \mathrm{~Hz}, 3.7 \mathrm{~Hz}, 1 \mathrm{H}$ ), 0.86-0.91 (m, 12H), $0.05(\mathrm{~s}, 6 \mathrm{H}) ;{ }^{13} \mathrm{C} \mathrm{NMR}\left(\mathrm{CDCl}_{3}, 100 \mathrm{MHz}\right) \delta 200.1,142.1,132.4,67.5,43.6$, 32.5, 25.8, 18.2, 18.1, 16.7, -5.5; IR (film) $v_{\max }$ 3363, 2958, 2931, 2887, 2858, 1472, 1256, 1096, 837; LRMS (ESI+) calcd. for $\mathrm{C}_{14} \mathrm{H}_{30} \mathrm{O}_{2} \mathrm{Si}[\mathrm{M}+\mathrm{Na}]^{+}$281.2, found: 281.1 .

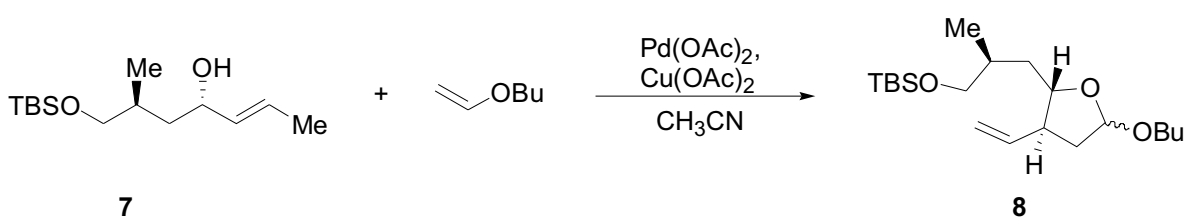

((S)-3-((2S,3S)-5-butoxy-3-vinyl-tetrahydrofuran-2-yl)-2-methylpropoxy)(tert-

butyl)dimethylsilane (8). To an 8 dram vial containing $750.0 \mathrm{mg}(2.901 \mathrm{mmol})$ of alcohol 4 was added $0.738 \mathrm{ml}(5.80 \mathrm{mmol})$ butyl vinyl ether and $2.9 \mathrm{ml}$ of acetonitrile. $65.1 \mathrm{mg}(0.290 \mathrm{mmol})$ of palladium (II) acetate and $1.318 \mathrm{~g}(7.254 \mathrm{mmol})$ of copper (II) acetate were then loaded into the vial. The reaction was purged with argon before being vigorously stirred for $15 \mathrm{~h}$ at $45^{\circ} \mathrm{C}$. At the end of this time period, the reaction was transferred to a $125 \mathrm{ml}$ Erlenmeyer flask, and was diluted with 50 $\mathrm{ml}$ of 3:1 hexanes:ether. The resultant precipitate was filtered, and the solvent from the eluent was then removed in vacuo. The crude oil was purified via flash silica chromatography (30:1 hexanes:EtOAc) yielding $181.9 \mathrm{mg}(0.5100 \mathrm{mmol}, 68 \%)$ of furan $\mathbf{8}$ as a 1:1 mixture of diastereomers at the acetal position and 1,2-induction of $\mathrm{dr}>20: 1$.

Diastereomer 1: $\mathrm{R}_{f}=0.54\left(10: 1\right.$ Hexanes:EtOAc); ${ }^{1} \mathrm{H}$ NMR $\left(\mathrm{CDCl}_{3}, 400 \mathrm{MHz}\right) \delta 5.72(\mathrm{ddd}, J=18.7$ $\mathrm{Hz}, 10.1 \mathrm{~Hz}, 8.6 \mathrm{~Hz}, 1 \mathrm{H}), 4.94-5.10(\mathrm{~m}, 3 \mathrm{H}), 3.74(\mathrm{dt}, J=9.1 \mathrm{~Hz}, 2.9 \mathrm{~Hz}, 1 \mathrm{H}), 3.66$ (dt, $J=9.7 \mathrm{~Hz}$, $6.8 \mathrm{~Hz}, 1 \mathrm{H}), 3.51(\mathrm{dd}, J=9.7 \mathrm{~Hz}, 5.4 \mathrm{~Hz}, 1 \mathrm{H}) 3.30-3.41(\mathrm{~m}, 2 \mathrm{H}), 2.21-2.40(\mathrm{~m}, 2 \mathrm{H}), 1.76-1.89$ (m, 
1H), 1.29-1.58 (m, 6H), 0.84-0.93 (m, 15H), $0.01(\mathrm{~s}, 6 \mathrm{H}) ;{ }^{13} \mathrm{C} \mathrm{NMR}\left(\mathrm{CDCl}_{3}, 125 \mathrm{MHz}\right) \delta 138.8$, 116.1, 103.3, 79.2, 68.8, 67.3, 50.0, 39.8, 39.5, 33.4, 31.8, 25.9, 19.4, 18.4, 16.5, 13.9, -5.4; IR (film) $v_{\max } 2958,2931,2860,1472,1256,1098,837$; LRMS (ESI+) calcd. for $\mathrm{C}_{20} \mathrm{H}_{40} \mathrm{O}_{3} \mathrm{Si}[\mathrm{M}+\mathrm{Na}]^{+} 379.3$, found: 379.3 .

Diastereomer 2: $\mathrm{R}_{f}=0.49$ (10:1 Hexanes:EtOAc); ${ }^{1} \mathrm{H}$ NMR $\left(\mathrm{CDCl}_{3}, 400 \mathrm{MHz}\right) \delta 5.65(\mathrm{ddd}, J=18.2$ $\mathrm{Hz}, 10.1 \mathrm{~Hz}, 8.3 \mathrm{~Hz}, 1 \mathrm{H}), 4.96-5.11(\mathrm{~m}, 3 \mathrm{H}), 3.76$ (dt, $J=9.7 \mathrm{~Hz}, 2.8 \mathrm{~Hz}, 1 \mathrm{H}), 3.66$ (dt, $J=9.5 \mathrm{~Hz}$, $6.8 \mathrm{~Hz}, 1 \mathrm{H}), 3.48(\mathrm{dd}, J=9.7 \mathrm{~Hz}, 5.8 \mathrm{~Hz}, 1 \mathrm{H}), 3.27-3.40(\mathrm{~m}, 2 \mathrm{H}), 2.61-2.72(\mathrm{~m}, 1 \mathrm{H}), 2.05(\mathrm{dd}, J=$ $12.6 \mathrm{~Hz}, 6.8 \mathrm{~Hz}, 1 \mathrm{H}), 1.82-1.92(\mathrm{~m}, 1 \mathrm{H}), 1.81(\mathrm{dt}, J=12.3 \mathrm{~Hz}, 4.9 \mathrm{~Hz}, 1 \mathrm{H}), 1.22-1.61(\mathrm{~m}, 6 \mathrm{H}), 0.85-$ $0.92(\mathrm{~m}, 15 \mathrm{H}), 0.01(\mathrm{~s}, 6 \mathrm{H}) ;{ }^{13} \mathrm{C} \mathrm{NMR}\left(\mathrm{CDCl}_{3}, 125 \mathrm{MHz}\right) \delta 138.5,116.0,103.2,82.1,68.6,66.7$, 48.3, 40.2, 36.7, 33.3, 31.9, 25.9, 19.4, 18.4, 16.2, 13.9, -5.4; IR (film) $v_{\max } 2958,2931,2860,1472$, 1256, 1098, 837; LRMS (ESI+) calcd. for $\mathrm{C}_{20} \mathrm{H}_{40} \mathrm{O}_{3} \mathrm{Si}[\mathrm{M}+\mathrm{Na}]^{+}$379.3, found: 379.3.

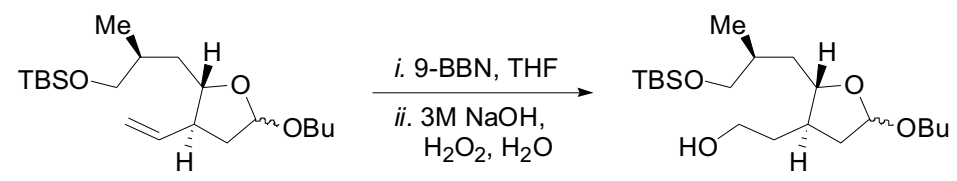

2-((2S,3R)-5-butoxy-2-((S)-3-(tert-butyldimethylsilyloxy)-2-methylpropyl)-tetrahydrofuran-3-

yl)ethanol. A flame-dried $100 \mathrm{ml} \mathrm{RBF}$ was charged with $738.4 \mathrm{mg}(2.071 \mathrm{mmol})$ of vinyl furan 5 and $20.7 \mathrm{ml}$ of THF. The solution was cooled to $0^{\circ} \mathrm{C}$ before $12.42 \mathrm{ml}(6.21 \mathrm{mmol})$ of a $0.50 \mathrm{M}$ of 9$\mathrm{BBN}$ was added. The reaction then stirred $3 \mathrm{~h}$ at this temperature before being quenched with $4.5 \mathrm{ml}$ of $3 \mathrm{M} \mathrm{NaOH}$ and $4.5 \mathrm{ml}$ of $30 \% \mathrm{H}_{2} \mathrm{O}_{2}$. After stirring for $2 \mathrm{~h}$, the excess peroxide was quenched by slowly adding saturated aqueous $\mathrm{Na}_{2} \mathrm{~S}_{2} \mathrm{O}_{3}$. The reaction was then extracted with ether, and the organic layers were washed with brine. The solution was dried over $\mathrm{MgSO}_{4}$, and the solvent was removed in vacuo. The crude oil was purified by flash silica chromatography (3:1 hexanes:EtOAc), affording $720.1 \mathrm{mg}$ (1.9222 mmol, 93\%) of alcohol as a mixture of diastereomers.

Diastereomer 1: $\mathrm{R}_{f}=0.34$ (3:1 Hexanes:EtOAc); ${ }^{1} \mathrm{H} \mathrm{NMR}\left(\mathrm{CDCl}_{3}, 400 \mathrm{MHz}\right) \delta 5.02(\mathrm{dd}, 5.6 \mathrm{~Hz}$, $2.3 \mathrm{~Hz}, 1 \mathrm{H}), 3.54-3.74(\mathrm{~m}, 4 \mathrm{H}), 3.49(\mathrm{dd}, J=9.7 \mathrm{~Hz}, 5.6 \mathrm{~Hz}, 1 \mathrm{H}), 3.28-3.37(\mathrm{~m}, 2 \mathrm{H}), 2.25(\mathrm{ddd}, J=$ $13.2 \mathrm{~Hz}, 7.7 \mathrm{~Hz}, 3.7 \mathrm{~Hz}, 1 \mathrm{H}), 1.93$ (bs), 1.40-1.87 (m, 8H), 1.26-1.37 (m, 3H), 0.83-0.91 (m, 15H), $0.01(\mathrm{~s}, 6 \mathrm{H}) ;{ }^{13} \mathrm{C} \mathrm{NMR}\left(\mathrm{CDCl}_{3}, 100 \mathrm{MHz}\right) \delta 103.3,80.2,68.7,67.1,61.4,40.8,38.8,37.8,35.5,33.3$, 31.8, 25.9, 19.4, 18.3, 16.4, 13.8, -5.4; IR (film) $v_{\max }$ 3396, 2956, 2931, 2860, 1472, 1252, 1096, 996, 837; LRMS (ESI+) calcd. for $\mathrm{C}_{20} \mathrm{H}_{42} \mathrm{O}_{4} \mathrm{Si}[\mathrm{M}+\mathrm{Na}]^{+}$397.3, found: 397.3.

Diastereomer 2: $\mathrm{R}_{f}=0.28$ (3:1 Hexanes:EtOAc); ${ }^{1} \mathrm{H}$ NMR $\left(\mathrm{CDCl}_{3}, 400 \mathrm{MHz}\right) \delta 5.00(\mathrm{~d}, \mathrm{~J}=4.9 \mathrm{~Hz}$, $1 \mathrm{H}), 3.72$ (ddd, $J=10.0 \mathrm{~Hz}, 7.4 \mathrm{~Hz}, 2.5 \mathrm{~Hz}, 1 \mathrm{H}), 3.59-3.69(\mathrm{~m}, 3 \mathrm{H}), 3.48$ (dd, $J=9.8 \mathrm{~Hz}, 5.9 \mathrm{~Hz}$, $1 \mathrm{H}), 3.36$ (dd, $J=9.8 \mathrm{~Hz}, 6.6 \mathrm{~Hz}, 1 \mathrm{H}), 3.30$ (dt, $J=9.5 \mathrm{~Hz}, 6.6 \mathrm{~Hz}, 1 \mathrm{H}), 2.03-2.16$ (m, 2H), $1.83-$ $1.95(\mathrm{~m}, 1 \mathrm{H}), 1.69-1.88(\mathrm{~m}, 1 \mathrm{H}), 1.43-1.64(\mathrm{~m}, 6 \mathrm{H}), 1.22-1.43(\mathrm{~m}, 4 \mathrm{H}), 0.86-0.92(\mathrm{~m}, 15 \mathrm{H}), 0.016(\mathrm{~s}$, $6 \mathrm{H}) ;{ }^{13} \mathrm{C} \mathrm{NMR}\left(\mathrm{CDCl}_{3}, 100 \mathrm{MHz}\right) \delta 103.4,83.1,68.9,66.7,61.8,40.4,40.1,39.9,36.1,33.3,31.8$, 26.0, 19.5, 18.4, 16.2, 13.9, -5.4; IR (film) $v_{\max } 3429,2956,2931,2858,1472,1256,1094,1065$, 837; LRMS (ESI+) calcd. for $\mathrm{C}_{20} \mathrm{H}_{42} \mathrm{O}_{4} \mathrm{Si}[\mathrm{M}+\mathrm{Na}]^{+}$397.3, found: 397.3. 


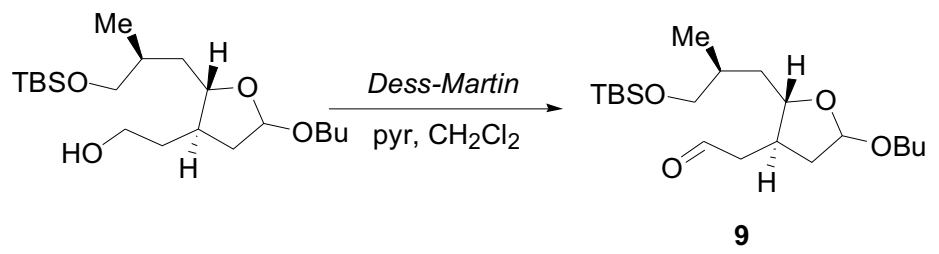

2-((2S,3R)-5-butoxy-2-((S)-3-(tert-butyldimethylsilyloxy)-2-methylpropyl)-tetrahydrofuran-3yl)acetaldehyde (9). A 2 dram vial was charged with $130.80 \mathrm{mg}(0.3491 \mathrm{mmol})$ of alcohol $\mathbf{5 a}$ and $3.49 \mathrm{ml}$ of $\mathrm{CH}_{2} \mathrm{Cl}_{2}$. The solution was cooled to $0{ }^{\circ} \mathrm{C}$, and $0.424 \mathrm{ml}(5.24 \mathrm{mmol})$ of pyridine was added, followed by $214.7 \mathrm{mg}(0.5063 \mathrm{mmol})$ Dess-Martin periodinane. The reaction stirred for $5 \mathrm{~min}$ before being warmed to room temperature for $50 \mathrm{~min}$. TLC revealed a small amount of $\mathbf{5 a}$ remaining, and therefore another $20 \mathrm{mg}(0.05 \mathrm{mmol})$ of periodinane was added. After stirring another 15 minutes, the reaction was quenched with a $1: 1$ solution of aqueous saturated $\mathrm{NaHCO}_{3}$ : aqueous saturated $\mathrm{Na}_{2} \mathrm{~S}_{2} \mathrm{O}_{3}$. After stirring the biphasic solution for 10 minutes, it was extracted with ether, and the organic layers were washed with brine and dried over $\mathrm{MgSO}_{4}$. After removing the solvent in vacuo, the crude oil was purified with flash silica chromatography (10:1 hexanes:EtOAc). $128.6 \mathrm{mg}$ $(0.3451 \mathrm{mmol}, 99 \%)$ of aldehyde 9 was isolated as a clear colorless oil. Note that clean separation of the two diastereomers was possible here, and therefore the two diastereomers were carried through separately.

Diastereomer 1: $\mathrm{R}_{f}=0.57\left(5: 1\right.$ Hexanes:EtOAc); ${ }^{1} \mathrm{H}$ NMR $\left(\mathrm{CDCl}_{3}, 400 \mathrm{MHz}\right) \delta 9.70(\mathrm{~s}, 1 \mathrm{H}), 5.01$ (dd, $J=5.4 \mathrm{~Hz}, 1.7 \mathrm{~Hz}, 1 \mathrm{H}), 3.68$ (ddd, $J=9.6 \mathrm{~Hz}, 7.7 \mathrm{~Hz}, 2.9 \mathrm{~Hz}, 1 \mathrm{H}), 3.58$ (dt, $J=9.6 \mathrm{~Hz}, 6.7 \mathrm{~Hz}$, $1 \mathrm{H}), 3.44$ (dd, $J=9.7 \mathrm{~Hz}, 5.8 \mathrm{~Hz}, 1 \mathrm{H}), 3.25-3.37(\mathrm{~m}, 2 \mathrm{H}), 2.48-2.64(\mathrm{~m}, 2 \mathrm{H}), 2.33$ (ddd, $J=15.0 \mathrm{~Hz}$, $9.8 \mathrm{~Hz}, 5.5 \mathrm{~Hz}, 1 \mathrm{H}), 2.03-2.15(\mathrm{~m}, 1 \mathrm{H}), 1.72-1.85(\mathrm{~m}, 1 \mathrm{H}), 1.42-1.52(\mathrm{~m}, 4 \mathrm{H}), 1.22-1.37(\mathrm{~m}, 3 \mathrm{H})$, 0.81-0.88 (m, 15H), $-0.03(\mathrm{~s}, 6 \mathrm{H}) ;{ }^{13} \mathrm{C} \mathrm{NMR}\left(\mathrm{CDCl}_{3}, 100 \mathrm{MHz}\right) \delta 201.0,103.3,80.0,68.5,67.0$, $47.8,38.8,37.7,33.1,31.8,25.9,19.3,18.2,16.3,13.8,-5.5$.

Diastereomer 2: $\mathrm{R}_{f}=0.50\left(0: 0\right.$ Hexanes:EtOAc); ${ }^{1} \mathrm{H}$ NMR $\left(\mathrm{CDCl}_{3}, 400 \mathrm{MHz}\right) \delta 9.72(\mathrm{t}, J=1.8 \mathrm{~Hz}$, $1 \mathrm{H}), 5.00(\mathrm{~d}, J=4.9 \mathrm{~Hz}, 1 \mathrm{H}), 3.71(\mathrm{ddd}, J=10.1 \mathrm{~Hz}, 7.6 \mathrm{~Hz}, 2.7 \mathrm{~Hz}, 1 \mathrm{H}), 3.63(\mathrm{dt}, J=9.5 \mathrm{~Hz}, 6.7$ $\mathrm{Hz}, 1 \mathrm{H}), 3.45(\mathrm{dd}, J=9.7 \mathrm{~Hz}, 5.9 \mathrm{~Hz}, 1 \mathrm{H}), 3.34(\mathrm{dd}, J=9.7 \mathrm{~Hz}, 6.5 \mathrm{~Hz}, 1 \mathrm{H}), 3.28$ (dt, $J=9.5 \mathrm{~Hz}, 6.5$ $\mathrm{Hz}, 1 \mathrm{H}), 2.55$ (ddd, $J=16.0 \mathrm{~Hz}, 4.9 \mathrm{~Hz}, 1.6 \mathrm{~Hz}, 1 \mathrm{H}), 2.42-2.52(\mathrm{~m}, 1 \mathrm{H}), 2.35$ (ddd, $J=16.0 \mathrm{~Hz}, 8.6$ $\mathrm{Hz}, 2.0 \mathrm{~Hz}, 1 \mathrm{H}), 2.18(\mathrm{dd}, J=12.7 \mathrm{~Hz}, 6.9 \mathrm{~Hz}, 1 \mathrm{H}), 1.81-1.91(\mathrm{~m}, 1 \mathrm{H}), 1.55-1.65(\mathrm{~m}, 2 \mathrm{H}), 1.43-1.53$ $(\mathrm{m}, 2 \mathrm{H}), 1.18-1.39(\mathrm{~m}, 3 \mathrm{H}), 0.84-0.89(\mathrm{~m}, 15 \mathrm{H}),-0.01(\mathrm{~s}, 6 \mathrm{H}) ;{ }^{13} \mathrm{C} \mathrm{NMR}\left(\mathrm{CDCl}_{3}, 100 \mathrm{MHz}\right) \delta 200.8$, $103.3,82.4,68.8,66.8,47.1,40.0,39.5,37.5,33.2,31.8,25.9,19.4,18.3,16.1,13.9,-5.4$.

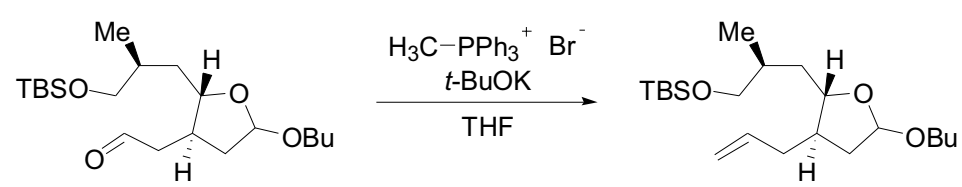

((S)-3-((2S,3R)-3-allyl-5-butoxy-tetrahydrofuran-2-yl)-2-methylpropoxy)(tert-

butyl)dimethylsilane. A 6 -dram vial was charged with $251.5 \mathrm{mg}(2.241 \mathrm{mmol}) t$-BuOK and 854.8 $\mathrm{mg}(2.393 \mathrm{mmol})$ methyltriphenylphosphonium bromide. $6.1 \mathrm{ml}$ of THF was added, and the resultant bright yellow solution was stirred for $45 \mathrm{~min}$ at room temperature. The activated Wittig reagent was then cooled to $0{ }^{\circ} \mathrm{C}$ and $297.2 \mathrm{mg}(0.7976 \mathrm{mmol})$ of aldehyde 9 in $2.4 \mathrm{ml}$ of THF was slowly added. The dark brown solution stirred for $2 \mathrm{~h}$ before it was quenched with $10 \%$ aqueous citric acid. The product was then extracted with ether, and the organic layers were washed with satd. aqueous $\mathrm{NaHCO}_{3}$ and brine. This solution was dried over $\mathrm{MgSO}_{4}$ and then the solvent was removed in vacuo. $287.2 \mathrm{mg}(0.7749 \mathrm{mmol})$ of the crude olefin was obtained. This material was clean enough to carry onto the next step unpurified. 
Diastereomer 1: $\mathrm{R}_{f}=0.65$ (10:1 Hexanes:EtOAc); ${ }^{1} \mathrm{H}$ NMR $\left(\mathrm{CDCl}_{3}, 400 \mathrm{MHz}\right) \delta 5.60-5.80(\mathrm{~m}, 1 \mathrm{H})$, 4.94-5.06 (m, 3H), 3.70 (ddd $J=9.8 \mathrm{~Hz}, 8.7 \mathrm{~Hz}, 2.8 \mathrm{~Hz}, 1 \mathrm{H}), 3.64$ (dt, $J=9.6 \mathrm{~Hz}, 6.8 \mathrm{~Hz}, 1 \mathrm{H}), 3.5$ $(\mathrm{dd}, J=9.7 \mathrm{~Hz}, 5.5 \mathrm{~Hz}, 1 \mathrm{H}), 3.31-3.37(\mathrm{~m}, 2 \mathrm{H}), 2.18-2.29(\mathrm{~m}, 2 \mathrm{H}), 2.02-2.12(\mathrm{~m}, 1 \mathrm{H}), 1.78-1.90(\mathrm{~m}$, $1 \mathrm{H}), 1.64-1.78(\mathrm{~m}, 1 \mathrm{H}), 1.30-1.58(\mathrm{~m}, 7 \mathrm{H}), 0.86-0.93(\mathrm{~m}, 15 \mathrm{H}), 0.02(\mathrm{~s}, 6 \mathrm{H}) ;{ }^{13} \mathrm{C} \mathrm{NMR}\left(\mathrm{CDCl}_{3}, 100\right.$ $\mathrm{MHz}) \delta 136.9,115.7,103.2,79.9,68.7,67.0,43.7,38.8,37.8,37.2,33.4,31.9,25.9,19.4,18.3,16.4$, 13.8, -5.4; IR (film) $v_{\max }$ 2958, 2929, 2858, 1472, 1256, 1096, 837; LRMS (ESI+) calcd. for $\mathrm{C}_{21} \mathrm{H}_{42} \mathrm{O}_{3} \mathrm{Si}[\mathrm{M}+\mathrm{Na}]^{+}$393.3, found: 393.2.

Diastereomer 2: $\mathrm{R}_{f}=0.54$ (10:1 Hexanes:EtOAc); ${ }^{1} \mathrm{H}$ NMR $\left(\mathrm{CDCl}_{3}, 400 \mathrm{MHz}\right) \delta 5.65-5.77(\mathrm{~m}, 1 \mathrm{H})$, 4.91-5.02 (m, 3H), 3.69 (ddd, $J=10.1 \mathrm{~Hz}, 7.1 \mathrm{~Hz}, 2.6 \mathrm{~Hz}, 1 \mathrm{H}), 3.63$ (dt, $J=9.5 \mathrm{~Hz}, 6.8 \mathrm{~Hz}, 1 \mathrm{H})$, 3.47 (dd, $J=9.7 \mathrm{~Hz}, 5.8 \mathrm{~Hz}, 1 \mathrm{H}), 3.34(\mathrm{dd}, J=9.7 \mathrm{~Hz}, 6.6 \mathrm{~Hz}, 1 \mathrm{H}), 3.27$ (dt, $J=9.5 \mathrm{~Hz}, 6.5 \mathrm{~Hz}, 1 \mathrm{H})$, 2.13-2.23 (m, 1H), 1.92-2.13 (m, 3H), 1.80-1.92 (m, 1H), 1.21-1.62 (m, 7H), 0.84-0.90 (m, 15H), $0.00(\mathrm{~s}, 6 \mathrm{H}) ;{ }^{13} \mathrm{C} \mathrm{NMR}\left(\mathrm{CDCl}_{3}, 100 \mathrm{MHz}\right) \delta 136.6,115.9,103.3,82.5,68.9,66.6,42.7,40.5,39.5$, 37.4, 33.3, 31.8, 25.9, 19.4, 18.3, 16.1, 13.9; IR (film) $v_{\max } 2956,2929,2858,1472,1256,1094,837$; LRMS (ESI+) calcd. for $\mathrm{C}_{21} \mathrm{H}_{42} \mathrm{O}_{3} \mathrm{Si}[\mathrm{M}+\mathrm{Na}]^{+}$393.3, found: 393.3 .

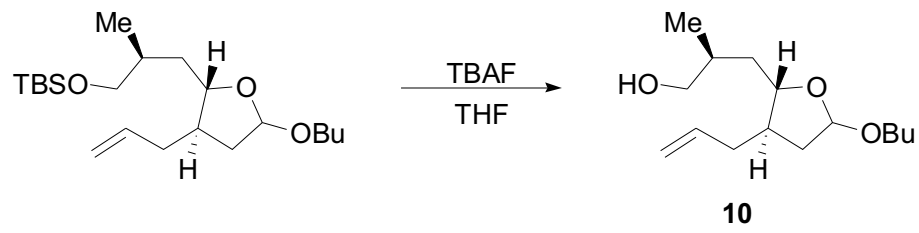

(S)-3-((2S,3R)-3-allyl-5-butoxy-tetrahydrofuran-2-yl)-2-methylpropan-1-ol (10). To a solution of crude alkene $(287.2 \mathrm{mg}, 0.7749 \mathrm{mmol})$ in dry THF $(7.75 \mathrm{ml})$ at $0{ }^{\circ} \mathrm{C}$ was added a $1 \mathrm{M}$ solution of tetrabutylammonium fluoride. $(1.55 \mathrm{ml}, 1.55 \mathrm{mmol})$ After 5 minutes the reaction was warmed to room temperature and was allowed to stir for $1 \mathrm{hr}$. At this time, TLC analysis revealed some remaining starting material, and therefore an additional equivalent $(0.77 \mathrm{ml}, 0.77 \mathrm{mmol})$ of tetrabutylammonium fluoride was added. After stirring an additional hour, the reaction was poured into saturated aqueous $\mathrm{NaHCO}_{3}$, and was extracted with ethyl acetate. The organic layers were combined and washed with saturated brine. The solution was dried with $\mathrm{MgSO}_{4}$, filtered, and concentrated in vacuo. Purification by flash chromatography (3.5:1 hexanes:EtOAc) afforded 173.2 $\mathrm{mg}(0.6756 \mathrm{mmol}, 85 \%$ over two steps $)$ of alcohol $\mathbf{1 0}$ as a clear, pale yellow oil.

Diastereomer 1: $\mathrm{R}_{f}=0.36$ (3:1 Hexanes:EtOAc); ${ }^{1} \mathrm{H}$ NMR $\left(\mathrm{CDCl}_{3}, 400 \mathrm{MHz}\right) \delta 5.67(\mathrm{~m}, 1 \mathrm{H}), 4.87$ $5.25(\mathrm{~m}, 3 \mathrm{H}), 3.64(\mathrm{dt}, J=9.2 \mathrm{~Hz}, 1.7 \mathrm{~Hz}, 1 \mathrm{H}), 3.56(\mathrm{dt}, J=9.5 \mathrm{~Hz}, 6.7 \mathrm{~Hz}, 1 \mathrm{H}), 3.42-3.51(\mathrm{bs}, 1 \mathrm{H})$, 3.23-3.37 (m, 3H), 2.12-2.25 (m, 2H), 1.96-2.06 (m, 1H), 1.59-1.79 (m, 2H), 1.41-1.54 (m, 4H), $1.23-1.40(\mathrm{~m}, 3 \mathrm{H}) ,0.85(\mathrm{~d}, J=6.8 \mathrm{~Hz}, 3 \mathrm{H}), 0.84(\mathrm{t}, J=7.4 \mathrm{~Hz}, 3 \mathrm{H}) ;{ }^{13} \mathrm{C} \mathrm{NMR}\left(\mathrm{CDCl}_{3}, 100 \mathrm{MHz}\right) \delta$ 136.4, 115.8, 103.4, 81.0, 68.3, 67.3, 43.7, 39.2, 38.6, 36.8, 34.7, 31.7, 19.2, 17.7, 13.6; IR (film) $v_{\max }$ 3452, 2958, 2931, 2873, 2734.1, 1449, 1098, 1030, 992, 913; LRMS (ESI+) calcd. for $\mathrm{C}_{15} \mathrm{H}_{28} \mathrm{O}_{3}[\mathrm{M}+$ $\mathrm{Na}]^{+} 279.2$, found: 279.1 .

Diastereomer 2: $\mathrm{R}_{f}=0.26$ (5:1 Hexanes:EtOAc); ${ }^{1} \mathrm{H}$ NMR $\left(\mathrm{CDCl}_{3}, 400 \mathrm{MHz}\right) \delta 5.68(\mathrm{~m}, 1 \mathrm{H}), 4.89$ $5.05(\mathrm{~m}, 3 \mathrm{H}), 3.64(\mathrm{ddd}, J=9.94 \mathrm{~Hz}, 7.59 \mathrm{~Hz}, 1.72 \mathrm{~Hz}, 1 \mathrm{H}), 3.59$ (dt, $J=9.4 \mathrm{~Hz}, 6.7 \mathrm{~Hz}, 1 \mathrm{H}), 3.44$ $(\mathrm{dd}, J=11.1 \mathrm{~Hz}, 4.6 \mathrm{~Hz}, 1 \mathrm{H}), 3.36(\mathrm{dd}, J=11.1 \mathrm{~Hz}, 6.9 \mathrm{~Hz}, 1 \mathrm{H}), 3.26(\mathrm{dt}, J=9.5 \mathrm{~Hz}, 6.5 \mathrm{~Hz}, 1 \mathrm{H})$, 3.20-3.50 (bs, $1 \mathrm{H}), 2.11-2.20(\mathrm{~m}, 1 \mathrm{H}), 1.89-2.09(\mathrm{~m}, 3 \mathrm{H}), 1.70-1.80(\mathrm{~m}, 1 \mathrm{H}), 1.34-1.61(\mathrm{~m}, 5 \mathrm{H})$, $1.22-1.34(\mathrm{~m}, 2 \mathrm{H}), 0.85(\mathrm{~d}, J=7.1 \mathrm{~Hz}, 3 \mathrm{H}),, 0.84(\mathrm{t}, J=7.5 \mathrm{~Hz}, 3 \mathrm{H}) ;{ }^{13} \mathrm{C} \mathrm{NMR}\left(\mathrm{CDCl}_{3}, 100 \mathrm{MHz}\right) \delta$ 136.3, 116.0, 103.4, 83.8, 68.6, 66.9, 42.7, 42.1, 39.1, 36.9, 35.1, 31.7, 19.3, 17.8, 13.8; IR (film) $\nu_{\max }$ 
3456, 2958, 2931, 2873, 1642, 1445, 1092, 1030, 884; LRMS (ESI+) calcd. for $\mathrm{C}_{15} \mathrm{H}_{28} \mathrm{O}_{3}[\mathrm{M}+\mathrm{Na}]^{+}$ 279.2, found: 279.1 .

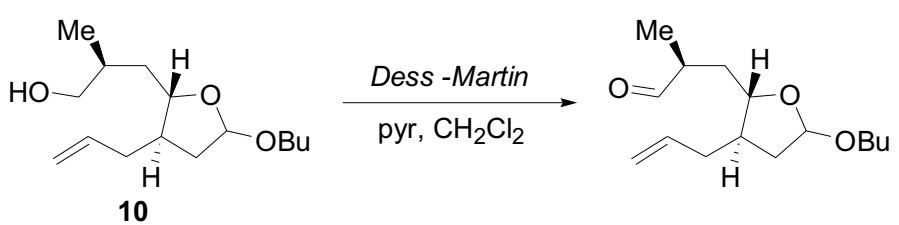

(S)-3-((2S,3R)-3-allyl-5-butoxy-tetrahydrofuran-2-yl)-2-methylpropanal. Alcohol 10 (134.60 $\mathrm{mg}, 0.525 \mathrm{mmol})$ was dissolved in dry $\mathrm{CH}_{2} \mathrm{Cl}_{2}(5.25 \mathrm{ml})$, and the resultant solution was cooled to 0 ${ }^{\circ} \mathrm{C}$. To this vessel, pyridine $(0.849 \mathrm{ml}, 10.5 \mathrm{mmol})$ and Dess-Martin periodinane $(334.0 \mathrm{mg}, 0.7875$ mmol) were successively added. After stirring for $10 \mathrm{~min}$, the reaction was warmed to room temperature for an additional $50 \mathrm{~min}$. It was then poured out into a 1:1 solution of aqueous saturated $\mathrm{NaHCO}_{3}$ and aqueous saturated $\mathrm{Na}_{2} \mathrm{~S}_{2} \mathrm{O}_{3}$. The biphasic solution was shaken for 3 minutes, before being extracted 3 times with $\mathrm{Et}_{2} \mathrm{O}$. The organic layers were combined, washed with brine, and were dried over $\mathrm{MgSO}_{4}$. After removing the solvent in vacuo, $133 \mathrm{mg}$ of the crude yellow oil was recovered. This material was carried on as a crude mixture.

Diastereomer 1: $\mathrm{R}_{f}=0.69$ (3:1 Hexanes:EtOAc); ${ }^{1} \mathrm{H}$ NMR $\left(\mathrm{CDCl}_{3}, 400 \mathrm{MHz}\right) \delta 9.57(\mathrm{~d}, J=1.89 \mathrm{~Hz}$, 1H) $5.60-5.72(\mathrm{~m}, 1 \mathrm{H}), 4.88-4.95(\mathrm{~m}, 3 \mathrm{H}), 3.64(\mathrm{ddd}, J=10.1 \mathrm{~Hz}, 7.97 \mathrm{~Hz}, 2.5 \mathrm{~Hz}, 1 \mathrm{H}), 3.53(\mathrm{dt}, J=$ $9.6 \mathrm{~Hz}, 6.8 \mathrm{~Hz}, 1 \mathrm{H}), 3.26(\mathrm{dt}, J=9.6 \mathrm{~Hz}, 6.4 \mathrm{~Hz}, 1 \mathrm{H}) 2.46-2.57(\mathrm{~m}, 1 \mathrm{H}) 2.12-2.24(\mathrm{~m}, 2 \mathrm{H}), 2.00-2.10$ $(\mathrm{m}, 1 \mathrm{H}), 1.64-1.81(\mathrm{~m}, 2 \mathrm{H}), 1.40-1.55(\mathrm{~m}, 4 \mathrm{H}), 1.23-1.36(\mathrm{~m}, 2 \mathrm{H}), 1.04(\mathrm{~d}, J=7.1 \mathrm{~Hz}, 3 \mathrm{H}), 0.834(\mathrm{t}$, $J=7.3 \mathrm{~Hz}, 3 \mathrm{H}) ;{ }^{13} \mathrm{C} \mathrm{NMR}\left(\mathrm{CDCl}_{3}, 100 \mathrm{MHz}\right) \delta 204.6,136.4,115.9,103.2,79.6,67.0,44.3,43.4$, $38.8,37.0,35.4,31.7,19.3,13.7,13.4$.

Diastereomer 2: $\mathrm{R}_{f}=0.58\left(10: 1\right.$ Hexanes:EtOAc); ${ }^{1} \mathrm{H}$ NMR $\left(\mathrm{CDCl}_{3}, 400 \mathrm{MHz}\right) \delta 9.61(\mathrm{~d}, J=1.3$ $\mathrm{Hz}, 1 \mathrm{H}), 5.62-5.75(\mathrm{~m}, 1 \mathrm{H}), 4.92-5.02(\mathrm{~m}, 3 \mathrm{H}), 3.67$ (ddd, $J=10.2 \mathrm{~Hz}, 6.9 \mathrm{~Hz}, 2.4 \mathrm{~Hz}, 1 \mathrm{H}), 3.57$ (dt, $J=9.4 \mathrm{~Hz}, 6.4 \mathrm{~Hz}, 1 \mathrm{H}), 2.51-2.62(\mathrm{~m}, 1 \mathrm{H}), 1.95-2.21(\mathrm{~m}, 4 \mathrm{H}), 1.85-1.94(\mathrm{~m}, 1 \mathrm{H}), 1.55-1.64(\mathrm{~m}, 1 \mathrm{H})$, 1.41-1.54 (m, 3H), 1.23-1.35 (m, 2H), $1.07(\mathrm{~d}, J=7.1 \mathrm{~Hz}, 3 \mathrm{H}), 0.85(\mathrm{t}, J=7.4 \mathrm{~Hz}, 3 \mathrm{H}) ;{ }^{13} \mathrm{C}$ NMR $\left(\mathrm{CDCl}_{3}, 100 \mathrm{MHz}\right) \delta 204.6,136.2,116.2,103.6,82.2,67.0,44.5,42.5,39.5,37.8,37.2,31.7,19.3$, 13.8, 13.2.

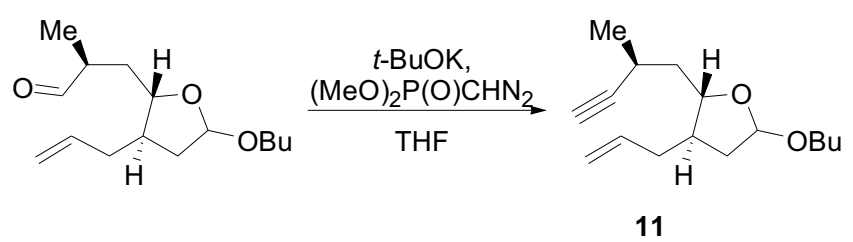

(2S,3R)-3-allyl-5-butoxy-2-((S)-2-methylbut-3-ynyl)-tetrahydrofuran (11). $\quad 88.4 \quad \mathrm{mg} \quad(0.788$ mmol) $t$-BuOK was dissolved in $6.3 \mathrm{ml} \mathrm{THF}$, and the resultant solution was cooled to $-78^{\circ} \mathrm{C}$. To the reaction vessel $1.39 \mathrm{ml}(0.814 \mathrm{mmol})$ of a $0.5865 \mathrm{M}$ solution of dimethyl (diazomethyl)phosphonate in THF was then slowly added. After stirring for $20 \mathrm{~min}$, all of the crude aldehyde $(\sim 0.525 \mathrm{mmol})$ was transferred to the reaction vessel using $1.34 \mathrm{ml} \mathrm{THF}$. The reaction stirred for $30 \mathrm{~min}$ at $-78{ }^{\circ} \mathrm{C}$ before it was warmed to room temperature. During this time, the color of the reaction turned from opaque yellow to clear, with the evolution of a deep orange precipitate. The reaction was quenched with 1:1 saturated aqueous ammonium chloride:water and was extracted with ether. The organic layers were neutralized with $\mathrm{NaHCO}_{3}$, washed with brine and were dried with $\mathrm{MgSO}_{4}$. After 
removing the solvent in vacuo, the crude oil was purified by silica gel chromatography (20:1 hexanes:EtOAc), affording $110.4 \mathrm{mg}(0.4410 \mathrm{mmol}, 84 \%$ for two steps $)$ of $\mathbf{1 1}$ as a clear colorless oil.

Diastereomer 1: $\mathrm{R}_{f}=0.68\left(10: 1\right.$ Hexanes:EtOAc); ${ }^{1} \mathrm{H}$ NMR $\left(\mathrm{CDCl}_{3}, 400 \mathrm{MHz}\right) \delta$ 5.67-5.79 $(\mathrm{m}, 1 \mathrm{H})$, 4.93-5.05 (m, 3H), $3.76(\mathrm{dt}, J=8.1 \mathrm{~Hz}, 4.0 \mathrm{~Hz}, 1 \mathrm{H}), 3.62(\mathrm{dt}, J=9.6 \mathrm{~Hz}, 6.8 \mathrm{~Hz}, 1 \mathrm{H}), 3.33(\mathrm{dt}, J=$ $9.6 \mathrm{~Hz}, 6.4 \mathrm{~Hz}, 1 \mathrm{H}), 2.57-2.68(\mathrm{~m}, 1 \mathrm{H}), 2.17-2.29(\mathrm{~m}, 2 \mathrm{H}), 2.05-2.14(\mathrm{~m}, 1 \mathrm{H}), 2.01(\mathrm{~d}, J=2.4 \mathrm{~Hz}$, $1 \mathrm{H}), 1.69-1.85(\mathrm{~m}, 2 \mathrm{H}), 1.46-1.59(\mathrm{~m}, 1 \mathrm{H}), 1.59-1.67(\mathrm{~m}, 3 \mathrm{H}), 1.28-1.40(\mathrm{~m}, 2 \mathrm{H}), 1.19(\mathrm{~d}, J=6.9$ $\mathrm{Hz}, 3 \mathrm{H}), 0.88$ (t, $J=7.3 \mathrm{~Hz}, 3 \mathrm{H}) .{ }^{13}{ }^{\mathrm{C}} \mathrm{NMR}\left(\mathrm{CDCl}_{3}, 100 \mathrm{MHz}\right) \delta 136.8,115.9,103.4,89.1,79.4$, 68.1, 67.1, 43.0, 41.0, 38.7, 37.4, 31.8, 22.6, 20.2, 19.4, 13.8; IR (film) $v_{\max } 3313,2960,2937,2917$, $2875,1642.0,1451.0,1098,1077,1025,982,631$; LRMS (ESI+) calcd. for $\mathrm{C}_{16} \mathrm{H}_{26} \mathrm{O}_{2}[\mathrm{M}+\mathrm{Na}]^{+}$ 273.2, found: 273.1 .

Diastereomer 2: $\mathrm{R}_{f}=0.62\left(10: 1\right.$ Hexanes:EtOAc); ${ }^{1} \mathrm{H}$ NMR $\left(\mathrm{CDCl}_{3}, 400 \mathrm{MHz}\right) \delta 5.66-5.78(\mathrm{~m}, 1 \mathrm{H})$, 4.93-5.04 (m, 3H), $3.76(\mathrm{dt}, J=7.4 \mathrm{~Hz}, 4.3 \mathrm{~Hz}, 1 \mathrm{H}), 3.64(\mathrm{dt}, J=9.5 \mathrm{~Hz}, 6.8 \mathrm{~Hz}, 1 \mathrm{H}), 3.29$ (dt, $J=$ $9.5 \mathrm{~Hz}, 6.4 \mathrm{~Hz}, 1 \mathrm{H}), 2.58-2.68(\mathrm{~m}, 1 \mathrm{H}), 2.08-2.26(\mathrm{~m}, 2 \mathrm{H}), 1.96-2.07(\mathrm{~m}, 3 \mathrm{H}), 1.74-1.86(\mathrm{~m}, 1 \mathrm{H})$, $1.55-1.67(\mathrm{~m}, 2 \mathrm{H}), 1.44-154(\mathrm{~m}, 2 \mathrm{H}), 1.27-1.38(\mathrm{~m}, 2 \mathrm{H}), 1.18(\mathrm{~d}, J=6.9 \mathrm{~Hz}, 3 \mathrm{H}), 0.87$ (t, $J=7.4 \mathrm{~Hz}$, $3 \mathrm{H}) ;{ }^{13} \mathrm{C}$ NMR $\left(\mathrm{CDCl}_{3}, 100 \mathrm{MHz}\right) \delta 136.4,116.1,103.5,89.1,81.9,68.1,66.8,43.5,42.2,39.5$, 37.6, 31.8, 22.7, 20.2, 19.4, 13.8; IR (film) $v_{\max } 3311,2960,2935,2875,1642.0,1445,1094,629$; LRMS (ESI+) calcd. for $\mathrm{C}_{16} \mathrm{H}_{26} \mathrm{O}_{2}[\mathrm{M}+\mathrm{Na}]^{+}$273.2, found: 273.1.

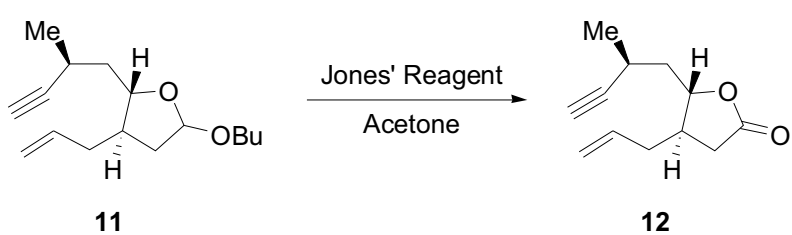

(4R,5S)-4-allyl-5-((S)-2-methylbut-3-ynyl)-dihydrofuran-2(3H)-one. $104.8 \mathrm{mg}(0.4187 \mathrm{mmol})$ of acetal 7 was charged into a 6 dram vial. The substrate was dissolved in $16.7 \mathrm{ml}$ acetone, and the resultant solution was cooled to $0{ }^{\circ} \mathrm{C}$ before $1.26 \mathrm{ml}(3.01 \mathrm{ml} / \mathrm{mmol})$ of Jones reagent was added. The reaction stirred for $30 \mathrm{~min}$ before it was quenched by the addition of 2-propanol $(1 \mathrm{ml})$. The blue-green precipitate was then filtered off, and the organic layer was neutralized by pouring into a large excess of aqueous sodium bicarbonate. The biphasic solution was extracted with EtOAc, and the organic layers were combined and washed with brine several times. The solution was then dried with $\mathrm{MgSO}_{4}$, and the solvent was removed in vacuo. The crude oil was purified with silica flash chromatography (6:1 hexanes:EtOAc), resulting in $73.4 \mathrm{mg}(0.3818 \mathrm{mmol}, 91 \%)$ of lactone $7 \mathbf{a}$ as a clear colorless oil. $\mathrm{R}_{f}=0.32(5: 1 \mathrm{Hexanes:EtOAc}) ;{ }^{1} \mathrm{H} \mathrm{NMR}\left(\mathrm{CDCl}_{3}, 400 \mathrm{MHz}\right) \delta$ 5.62-5.74 (m, $1 \mathrm{H}), 5.02-5.10(\mathrm{~m}, 2 \mathrm{H}), 4.29(\mathrm{dt}, J=7.5 \mathrm{~Hz}, 5.4 \mathrm{~Hz}, 1 \mathrm{H}), 2.56-2.68(\mathrm{~m}, 2 \mathrm{H}), 2.17-2.33(\mathrm{~m}, 3 \mathrm{H}), 2.06-$ $2.17(\mathrm{~m}, 1 \mathrm{H}), 2.05(\mathrm{~d}, J=2.4 \mathrm{~Hz}, 1 \mathrm{H}), 1.88$ (ddd, $J=14.0 \mathrm{~Hz}, 7.9 \mathrm{~Hz}, 6.7 \mathrm{~Hz}, 1 \mathrm{H}), 1.72$ (ddd, $J=$ $14.2 \mathrm{~Hz}, 7.2 \mathrm{~Hz}, 4.8 \mathrm{~Hz}, 1 \mathrm{H}), 1.20(\mathrm{~d}, J=6.9 \mathrm{~Hz}, 3 \mathrm{H}) ;{ }^{13} \mathrm{C} \mathrm{NMR}\left(\mathrm{CDCl}_{3}, 100 \mathrm{MHz}\right) \delta 176.0,134.2$, 117.9, 87.5, 82.2, 69.2, 40.8, 40.0, 36.9, 34.1, 22.1, 20.1; IR (film) $v_{\max } 3294,2977,2935,1777$, 1169, 973; LRMS (ESI+) calcd. for $\mathrm{C}_{12} \mathrm{H}_{16} \mathrm{O}_{2}[\mathrm{M}+\mathrm{Na}]^{+}$215.1, found: 215.0 . 


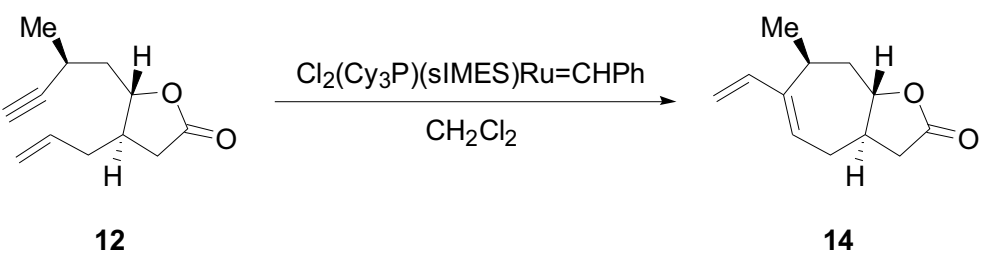

(3aR,7S,8aS,Z)-7-methyl-6-vinyl-3a,4,8,8a-tetrahydro-3H-cyclohepta[b]furan-2(7H)-one (14). A flame-dried $100 \mathrm{ml}$ RBF was charged with $10.8 \mathrm{mg}(0.01212 \mathrm{mmol})$ of tricyclohexylphosphine [1,3-bis(2,4,6-trimethylphenyl)-4,5-dihydroimidazol-2-ylidene][bezylidine]-ruthenium dichloride. The metal was dissolved in $40 \mathrm{ml} \mathrm{CH}_{2} \mathrm{Cl}_{2}$. $46.6 \mathrm{mg}(0.2424 \mathrm{mmol})$ of eneyne $7 \mathbf{a}$ was then transferred into the reaction vessel using $8.5 \mathrm{ml}$ of $\mathrm{CH}_{2} \mathrm{Cl}_{2}$. The resultant light pink solution slowly darkened to a dark purple over the course of 3 hours. The reaction was then quenched by sparging with air, and the solvent was removed in vacuo. The crude oil was then purified by silica chromatography (6:1 Hex:EtOAc). The reaction afforded $32.4 \mathrm{mg}(0.1684 \mathrm{mmol}, 70 \%)$ of diene 8 as an oil which upon prolonged sitting formed white crystals. Note that $10 \%$ of eneyne $7 \mathbf{a}$ was also recovered. $\mathrm{R}_{f}=0.27\left(5: 1\right.$ Hexanes:EtOAc); ${ }^{1} \mathrm{H}$ NMR $\left(\mathrm{CDCl}_{3}, 400 \mathrm{MHz}\right) \delta 6.20(\mathrm{dd}, J=17.4 \mathrm{~Hz}, 5.4$ $\mathrm{Hz}, 1 \mathrm{H}), 5.75$ (dd, $J=8.8 \mathrm{~Hz}, 2.5 \mathrm{~Hz}, 1 \mathrm{H}), 5.15$ (d, $J=17.4 \mathrm{~Hz}, 1 \mathrm{H}), 4.94(\mathrm{~d}, J=10.8 \mathrm{~Hz}, 1 \mathrm{H}), 4.33$ (ddd, $J=12.1 \mathrm{~Hz}, 9.7 \mathrm{~Hz}, 2.8 \mathrm{~Hz}, 1 \mathrm{H}), 3.00-3.10$ (m, 1H), 2.56 (dd, $J=16.6 \mathrm{~Hz}, 6.6 \mathrm{~Hz}, 1 \mathrm{H}), 2.44$ (ddd, $J=15.5 \mathrm{~Hz}, 9.2 \mathrm{~Hz}, 1.6 \mathrm{~Hz}, 1 \mathrm{H}), 2.24-2.37$ (m, 2H), 1.98-2.15 (m, 2H), 1.71 (dt, $J=12.5 \mathrm{~Hz}$, $3.8 \mathrm{~Hz}, 1 \mathrm{H}), 1.12(\mathrm{~d}, J=7.5 \mathrm{~Hz}, 3 \mathrm{H}) ;{ }^{13} \mathrm{C} \mathrm{NMR}\left(\mathrm{CDCl}_{3}, 100 \mathrm{MHz}\right) \delta 175.7,145.6,141.4,129.5$, 110.5, 84.0, 44.2, 36.6, 36.4, 28.6, 28.3, 18.5; IR (film) $v_{\max } 2968$, 2933, 1785, 1204, 976; LRMS (ESI+) calcd. for $\mathrm{C}_{12} \mathrm{H}_{16} \mathrm{O}_{2}[\mathrm{M}+\mathrm{Na}]^{+} 215.1$, found: 215.1 .

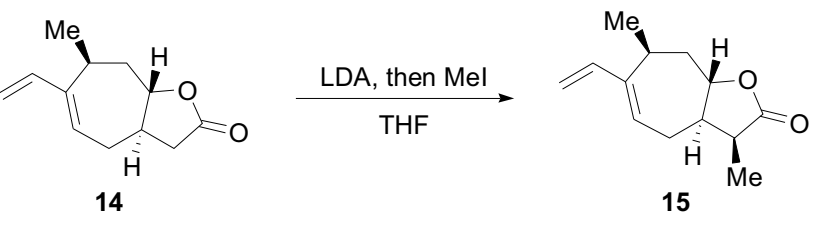

(3S,3a $R, 7 S, 8 \mathrm{a} S, Z)-3,7-d i m e t h y l-6-v i n y l-3 a, 4,8,8 \mathrm{a}-t e t r a h y d r o-3 H-c y c l o h e p t a[b] f u r a n-2(7 \mathrm{H})-o n e$ (15). A $0.500 \mathrm{M}$ stock solution of LDA was made by deprotonating $0.735 \mathrm{ml}(5.24 \mathrm{mmol})$ diisopropyl amine with $2.03 \mathrm{ml}(5.00 \mathrm{mmol})$ of $2.46 \mathrm{M} n$-BuLi in hexanes. This was setup at $-78{ }^{\circ} \mathrm{C}$ in $7.23 \mathrm{ml} \mathrm{THF}$ and after stirring $5 \mathrm{~min}$, it was warmed to $0{ }^{\circ} \mathrm{C}$. After 20 minutes, $49.9 \mu 1(0.111$ $\mathrm{mmol})$ of the stock LDA solution was added to $21.4 \mathrm{mg}(0.111 \mathrm{mmol})$ of lactone 8 in $0.89 \mathrm{ml}$ of THF at $-78{ }^{\circ} \mathrm{C}$. The reaction stirred for $1 \mathrm{~h}$ before adding $27.7 \mu \mathrm{l}(0.445 \mathrm{mmol})$ of freshly distilled iodomethane. After stirring at $-78{ }^{\circ} \mathrm{C}$ for 50 minutes, the reaction was warmed to room temperature and stirred an additional $3 \mathrm{~h}$. At this time it was quenched with satd. aqueous $\mathrm{NaHCO}_{3}$. The product was extracted with diethyl ether, and the organic layers were then washed with brine and dried with $\mathrm{MgSO}_{4}$. The solvent was then removed in vacuo, and the crude oil was purified via flash silica chromatography (6:1 hexanes:EtOAc). $20.7 \mathrm{mg}(0.1003 \mathrm{mmol}, 90 \%)$ of the desired alkylated lactone 9 was obtained as a white crystalline material in greater than 20:1 diastereoselectivity. $\mathrm{R}_{f}=0.35(5: 1$ Hexanes:EtOAc); ${ }^{1} \mathrm{H}$ NMR $\left(\mathrm{CDCl}_{3}, 400 \mathrm{MHz}\right) \delta 6.21(\mathrm{dd}, J=17.4 \mathrm{~Hz}, 10.9 \mathrm{~Hz}, 1 \mathrm{H}), 5.78(\mathrm{~m}, 1 \mathrm{H})$, $5.11(\mathrm{~d}, J=17.5 \mathrm{~Hz}, 1 \mathrm{H}), 4.94(\mathrm{~d}, J=10.9 \mathrm{~Hz}, 1 \mathrm{H}), 4.50$ (ddd, $J=13.3 \mathrm{~Hz}, 10.0 \mathrm{~Hz}, 2.92 \mathrm{~Hz}, 1 \mathrm{H})$, 3.00-3.10 (m, 1H), 2.66 (dq, $J=7.7 \mathrm{~Hz}, 7.6 \mathrm{~Hz}, 1 \mathrm{H}), 2.21-2.33(\mathrm{~m}, 2 \mathrm{H}), 2.02-2.16(\mathrm{~m}, 2 \mathrm{H}), 1.67$ (dt, $J=12.4 \mathrm{~Hz}, 3.7 \mathrm{~Hz}, 1 \mathrm{H}), 1.19(\mathrm{~d}, J=7.8 \mathrm{~Hz}, 3 \mathrm{H}), 1.11(\mathrm{~d}, J=7.5 \mathrm{~Hz}, 3 \mathrm{H}) ;{ }^{13} \mathrm{C} \mathrm{NMR}\left(\mathrm{CDCl}_{3}, 100\right.$ MHz) $\delta 179.4,145.4,141.3,129.7,110.3,81.4,46.2,40.0,36.5,28.1,24.9$, 18.3, 10.1; IR (film) $v_{\max }$ 2971, 2935, 1779, 1206, 990; LRMS (ESI+) calcd. for $\mathrm{C}_{13} \mathrm{H}_{18} \mathrm{O}_{2}[\mathrm{M}+\mathrm{H}]^{+}$207.1, found: 207.1. 


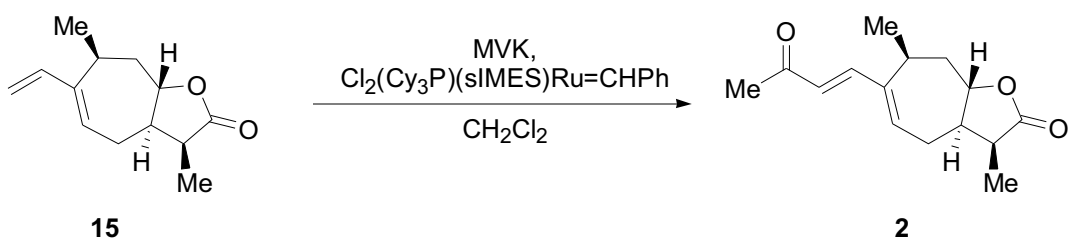

(11, ,13-dihydroxanthatin) (2). A $5 \mathrm{~mm}$ NMR tube was charged with $5.1 \mathrm{mg}(0.025 \mathrm{mmol})$ of diene 9 and $3.4 \mathrm{mg}(0.049 \mathrm{mmol})$ methyl vinyl ketone. $2.2 \mathrm{mg}(2.5 \mu \mathrm{mol})$ tricyclohexylphosphine [1,3-bis(2,4,6-trimethylphenyl)-4,5-dihydroimidazol-2-ylidene] [bezylidine]-ruthenium dichloride was then dissolved in $0.759 \mathrm{ml}$ of $d 2-\mathrm{CH}_{2} \mathrm{Cl}_{2}$ which was subsequently transferred to the NMR tube. The reaction was monitored by NMR for $3 \mathrm{~h}$, by which time it appeared to have gone to $70 \%$ conversion. The solvent was removed in vacuo, and the crude oil was purified by flash silica chromatography (1:1 pentane:ether). $4.0 \mathrm{mg}(0.1611 \mathrm{mmol}, 66 \%)$ of a clear colorless oil was obtained. This material (shown to be the desired compound 2 ) could be recrystallized from a solution of pentane and chloroform to afford white needlelike crystals. $\mathrm{R}_{f}=0.38$ (1:1 Hexanes:EtOAc); $[\alpha]_{\mathrm{D}}{ }^{25}=-49.0^{\circ}\left(\mathrm{CHCl}_{3}, c=0.83\right) ;{ }^{1} \mathrm{H} \mathrm{NMR}\left(\mathrm{CDCl}_{3}, 500 \mathrm{MHz}\right) \delta 7.03(\mathrm{~d}, J=16.1 \mathrm{~Hz}, 1 \mathrm{H}), 6.23(\mathrm{dd}$, $J=9.3 \mathrm{~Hz}, 3.4 \mathrm{~Hz}, 1 \mathrm{H}), 6.15(\mathrm{~d}, 16.1 \mathrm{~Hz}, 1 \mathrm{H}), 4.51(\mathrm{ddd}, J=11.9 \mathrm{~Hz}, 10.4 \mathrm{~Hz}, 2.9 \mathrm{~Hz}, 1 \mathrm{H}), 2.97-$ $3.05(\mathrm{~m}, 1 \mathrm{H}) 2.69(\mathrm{qu}, J=7.7 \mathrm{~Hz}, 1 \mathrm{H}), 2.42(\mathrm{ddd}, J=16.0 \mathrm{~Hz}, 9.3 \mathrm{~Hz}, 2.0 \mathrm{~Hz}, 1 \mathrm{H}), 2.32(\mathrm{dt}, J=$ $13.0 \mathrm{~Hz}, 3.4 \mathrm{~Hz}, 1 \mathrm{H}), 2.27(\mathrm{~s}, 3 \mathrm{H}), 2.05-2.23(\mathrm{~m}, 2 \mathrm{H}), 1.69(\mathrm{dt}, J=12.4 \mathrm{~Hz}, 3.7 \mathrm{~Hz}, 1 \mathrm{H}), 1.20$ (d, $J=$ $7.8 \mathrm{~Hz}, 3 \mathrm{H}), 1.13(\mathrm{~d}, J=7.5 \mathrm{~Hz}, 3 \mathrm{H}) ;{ }^{13} \mathrm{C} \mathrm{NMR}\left(\mathrm{CDCl}_{3}, 125 \mathrm{MHz}\right) \delta 198.5,179.0,148.3,144.5$, $139.2,124.4,80.8,45.8,39.8,36.3,29.0,27.8,25.7,18.3$, 10.1; IR (film) $v_{\max } 2970,2931,2879$, 1779, 1663, 1590, 1457, 1258, 1207, 984; LRMS (ESI+) calcd. for $\mathrm{C}_{15} \mathrm{H}_{20} \mathrm{O}_{3}[\mathrm{M}+\mathrm{H}]^{+} 249.2$, found: 249.1 .

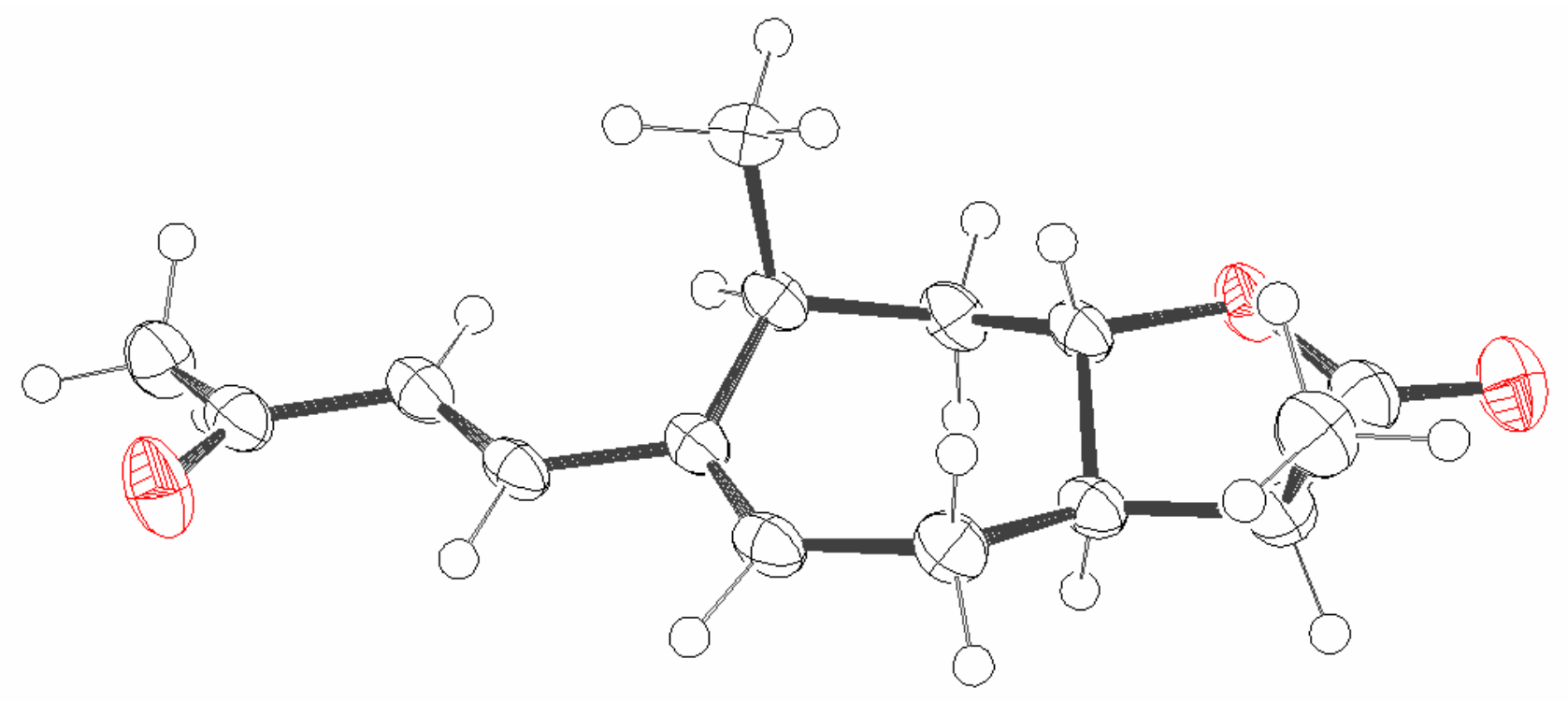




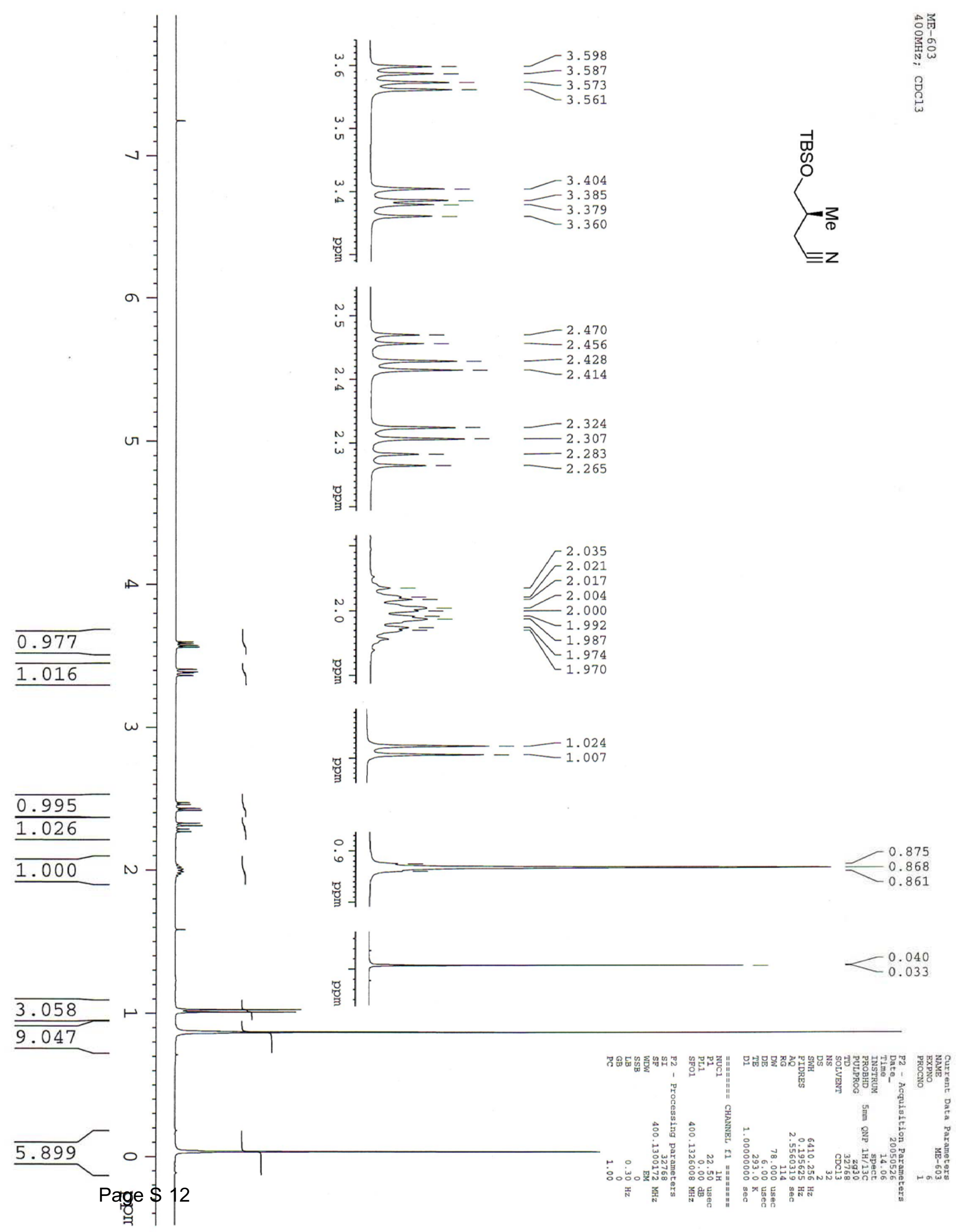




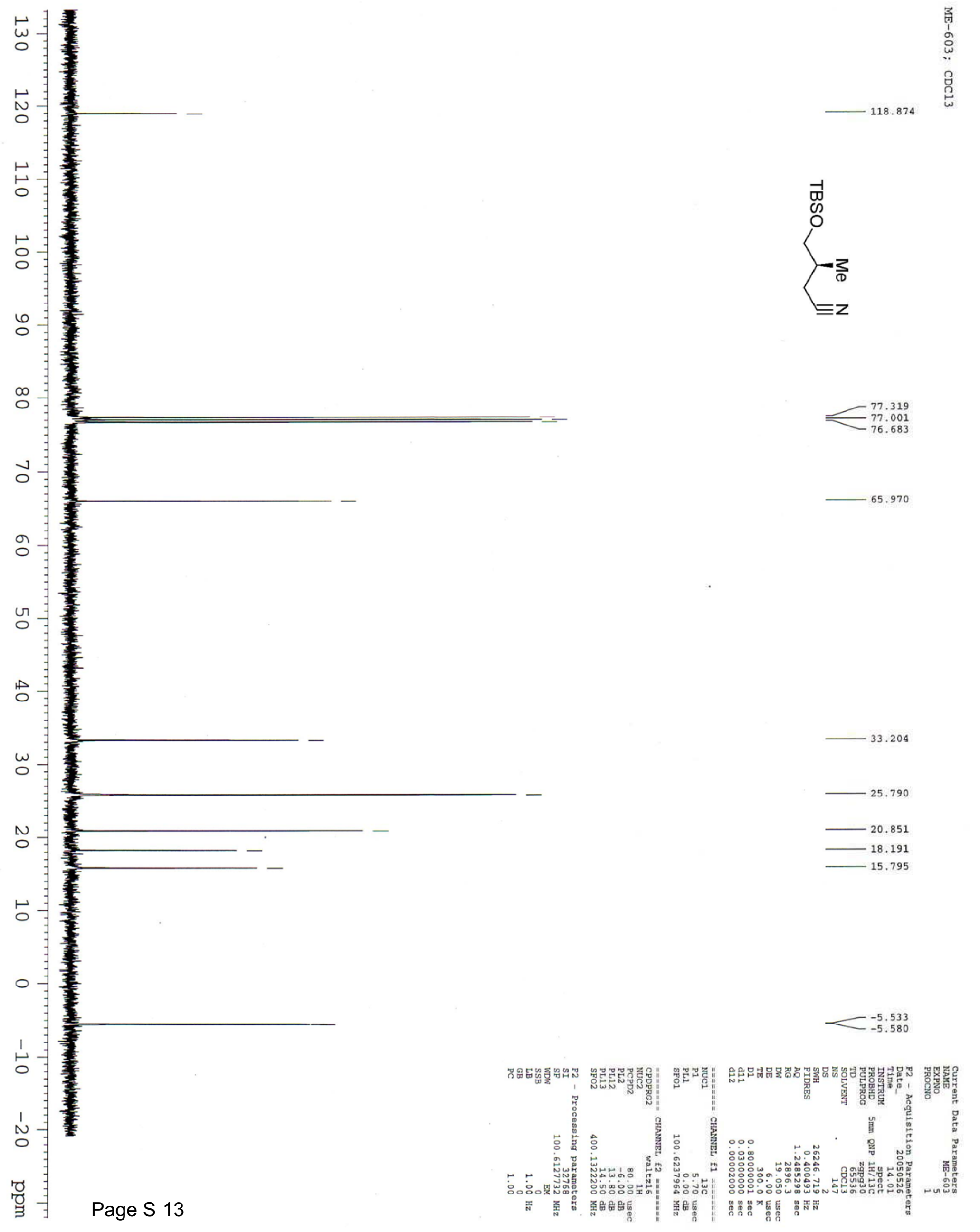




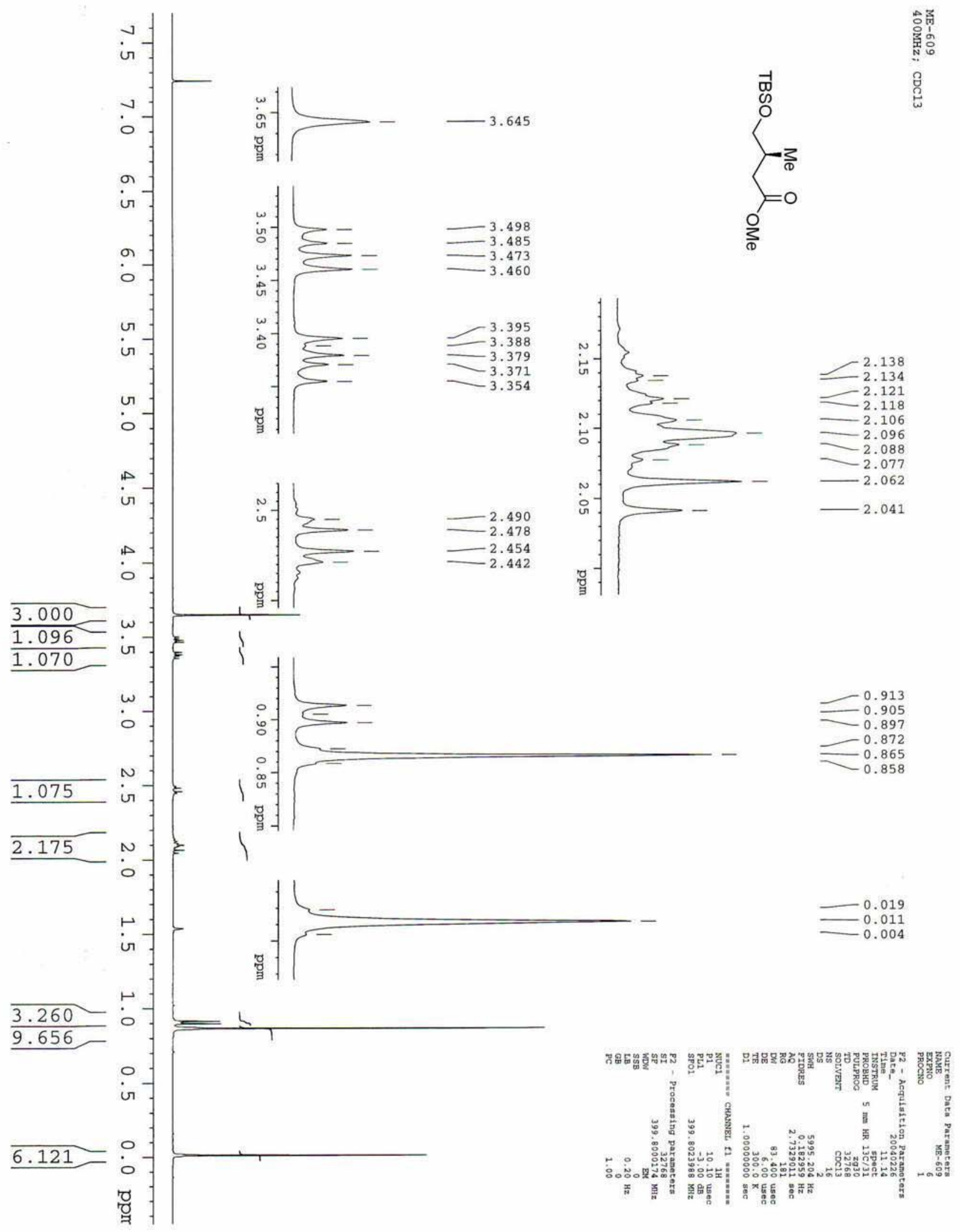




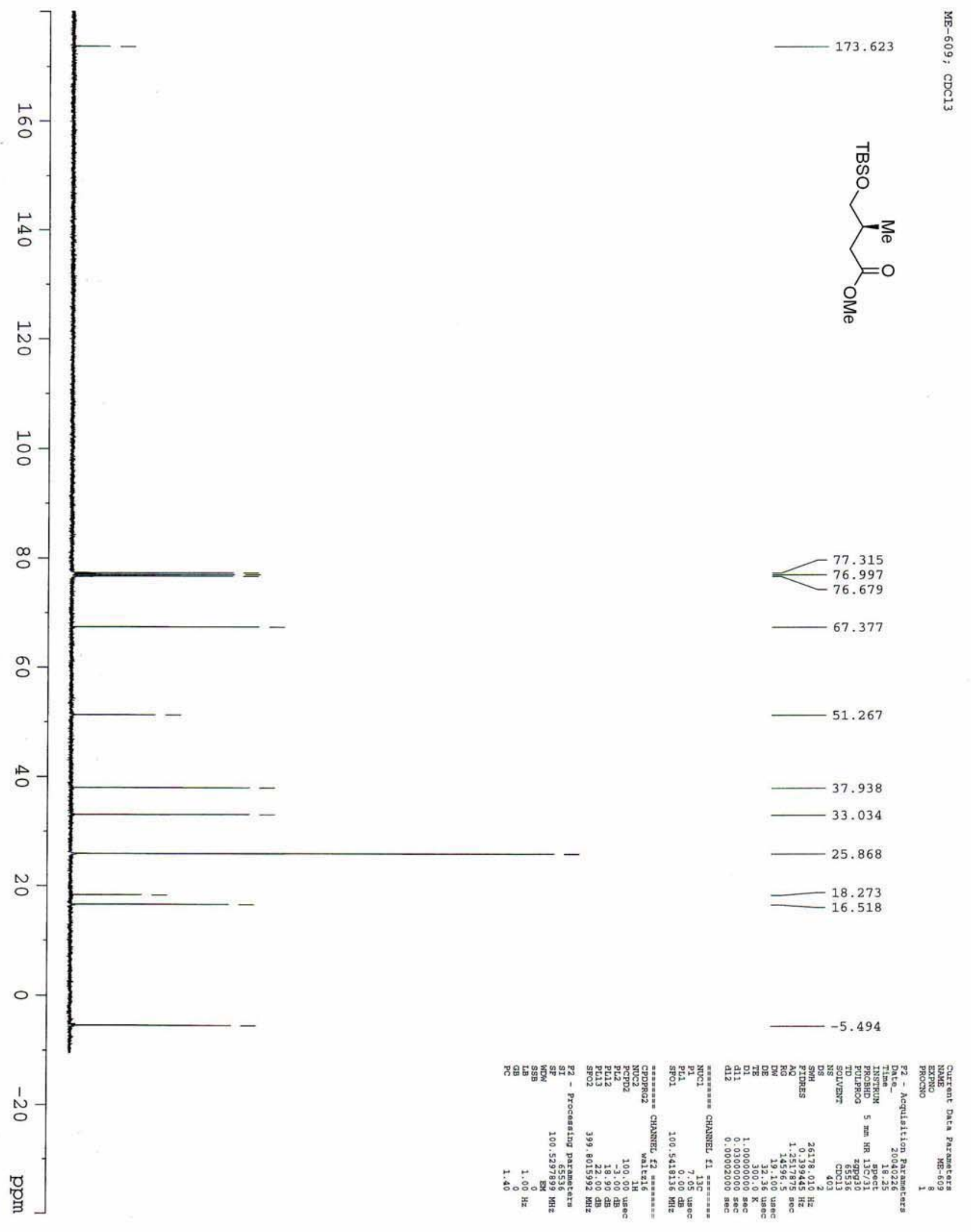

Page S 15 


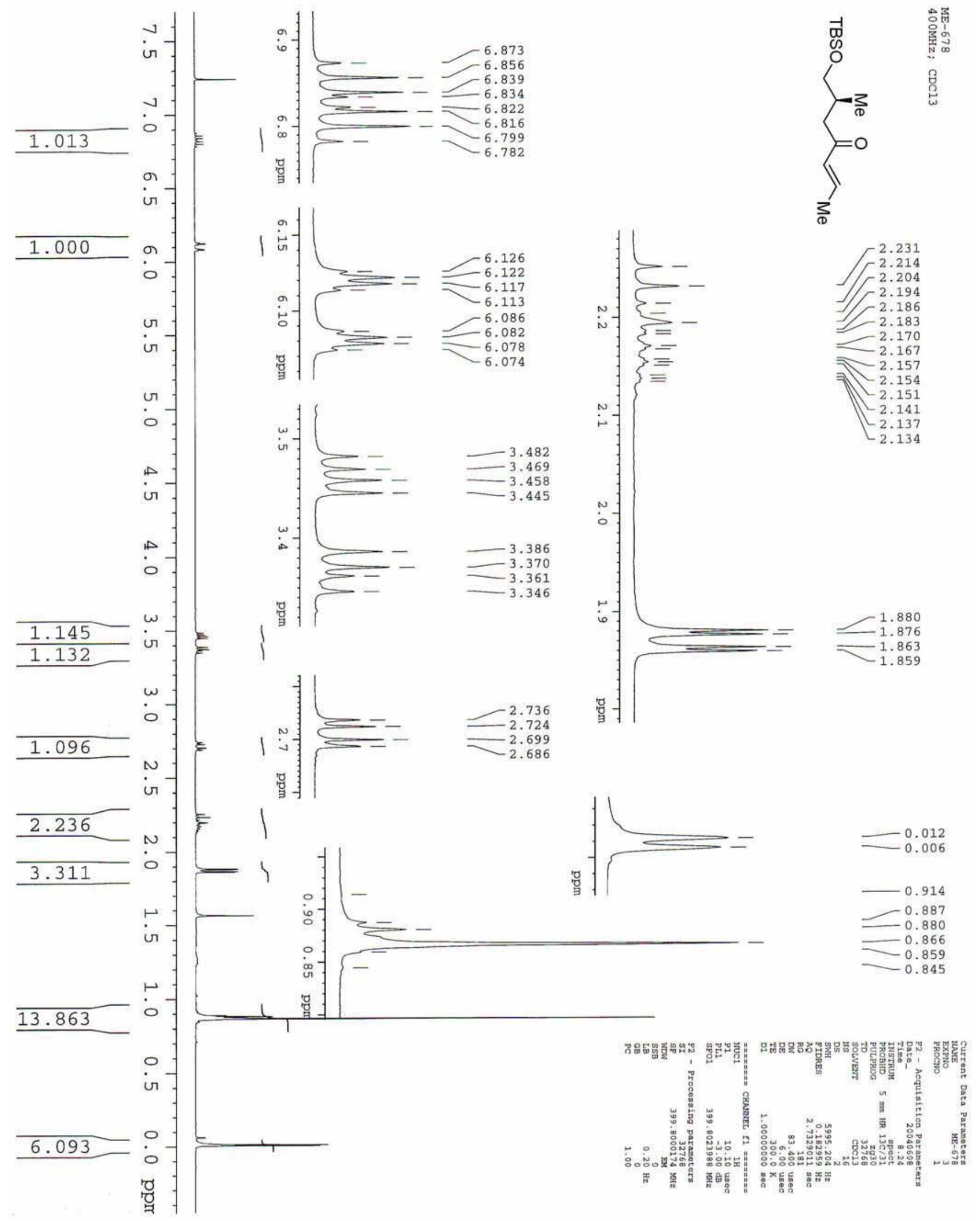




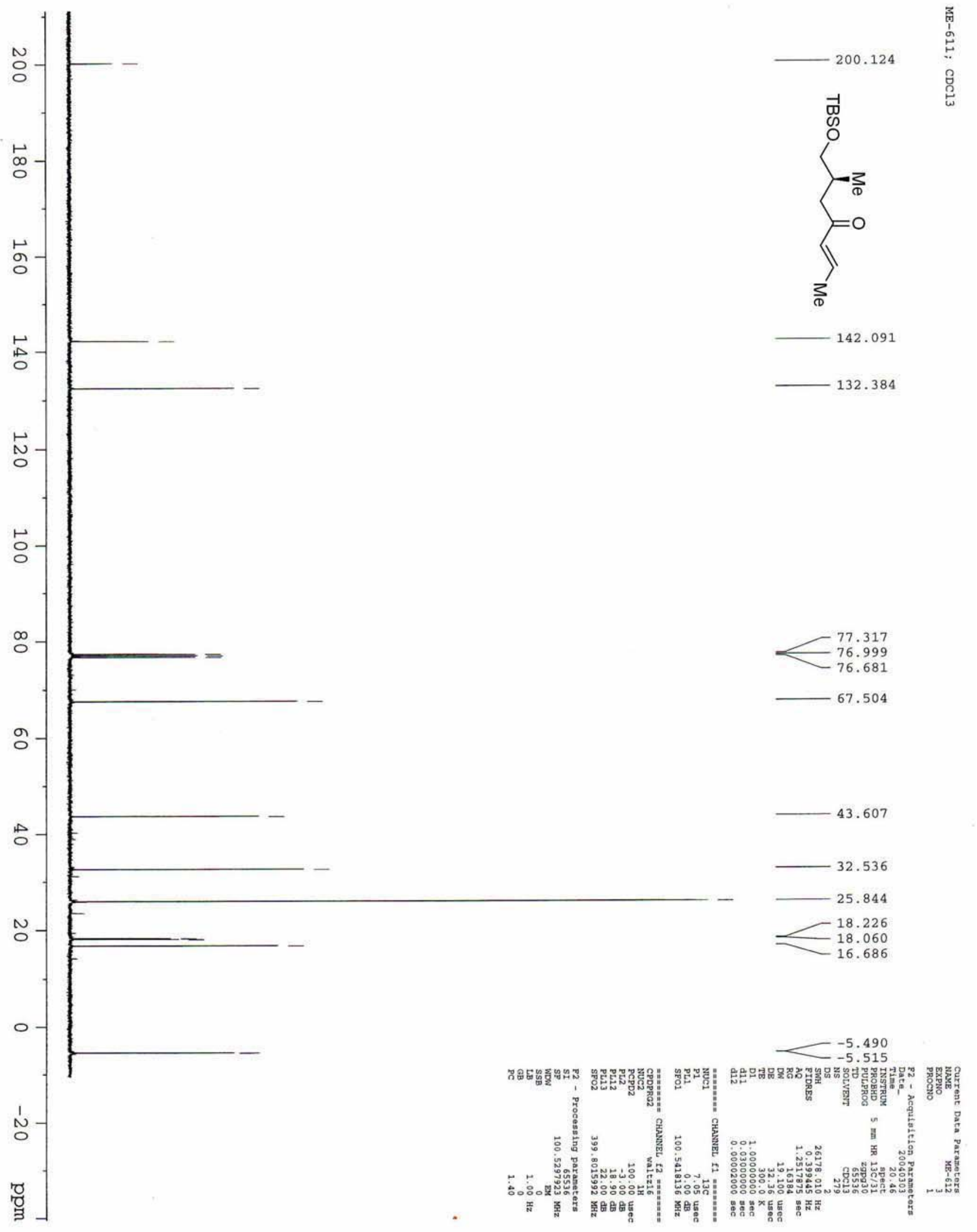




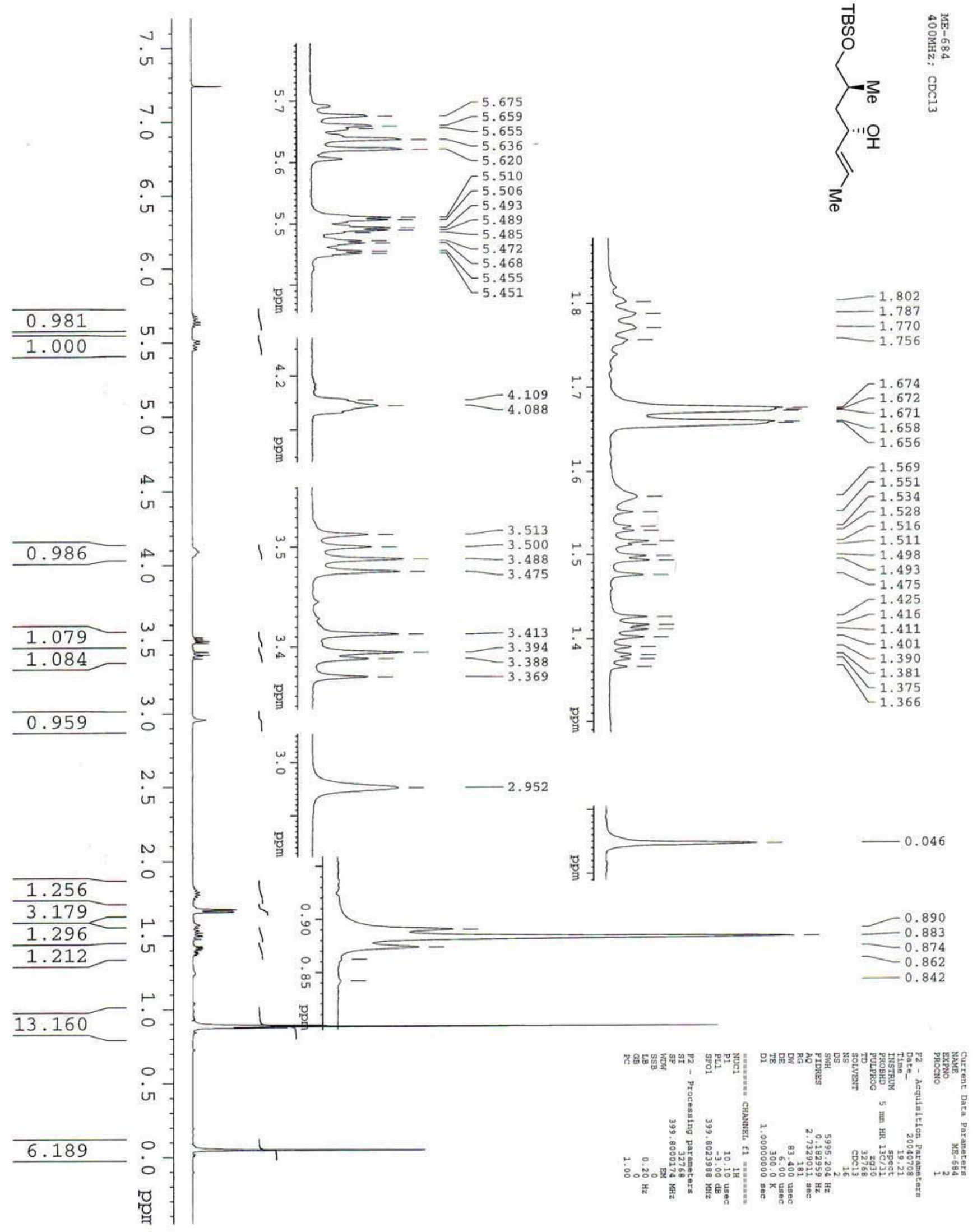




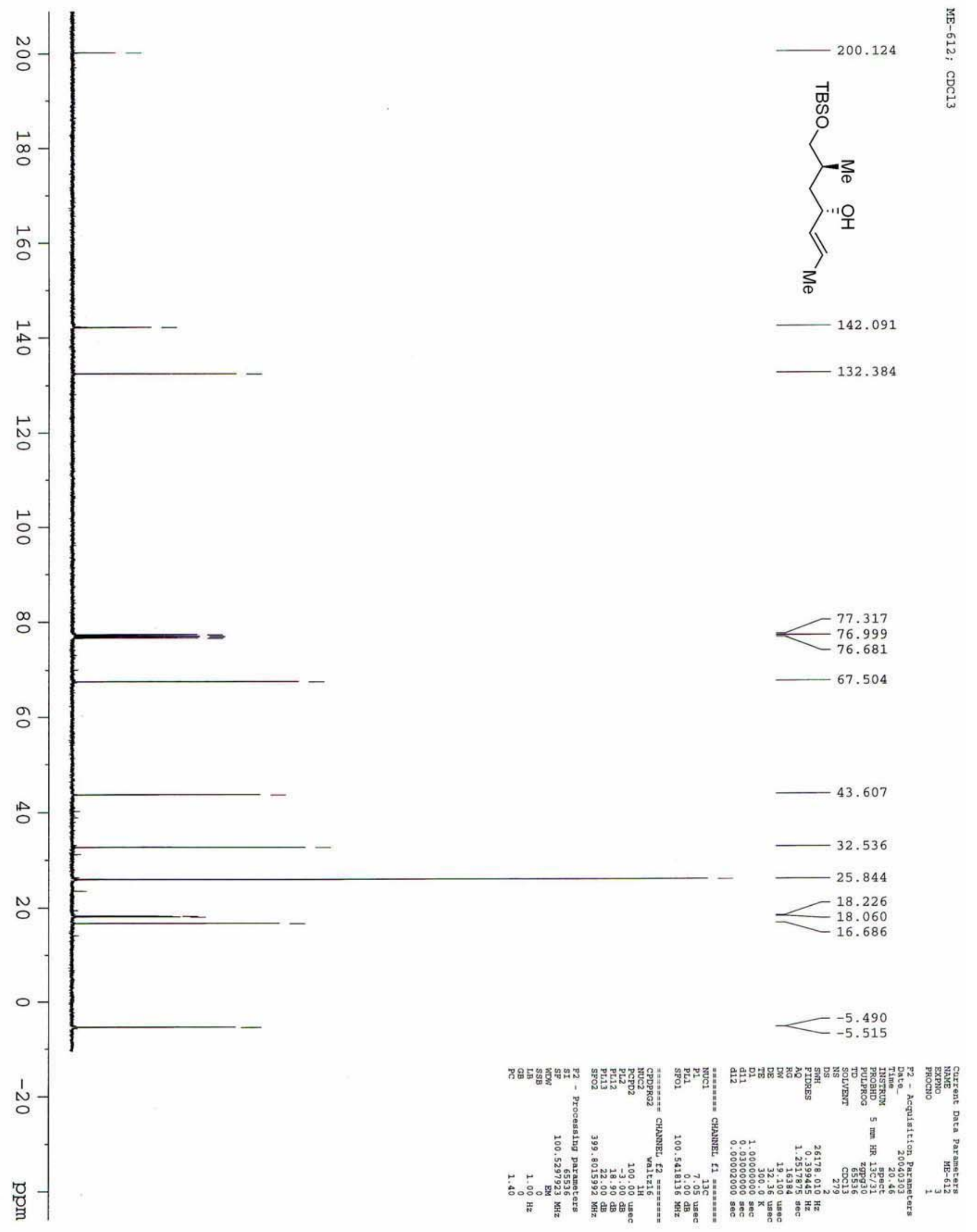

Page S 19 


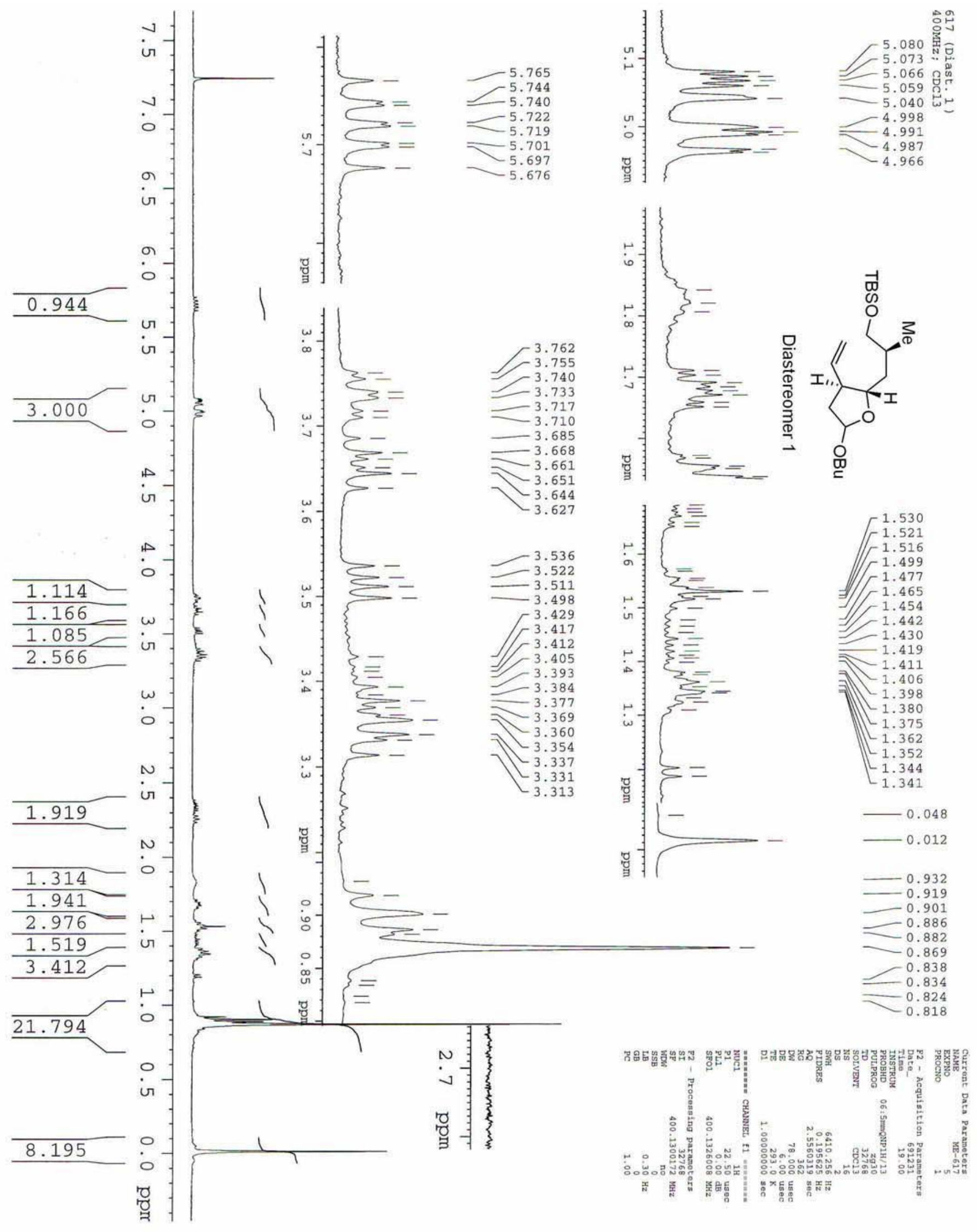




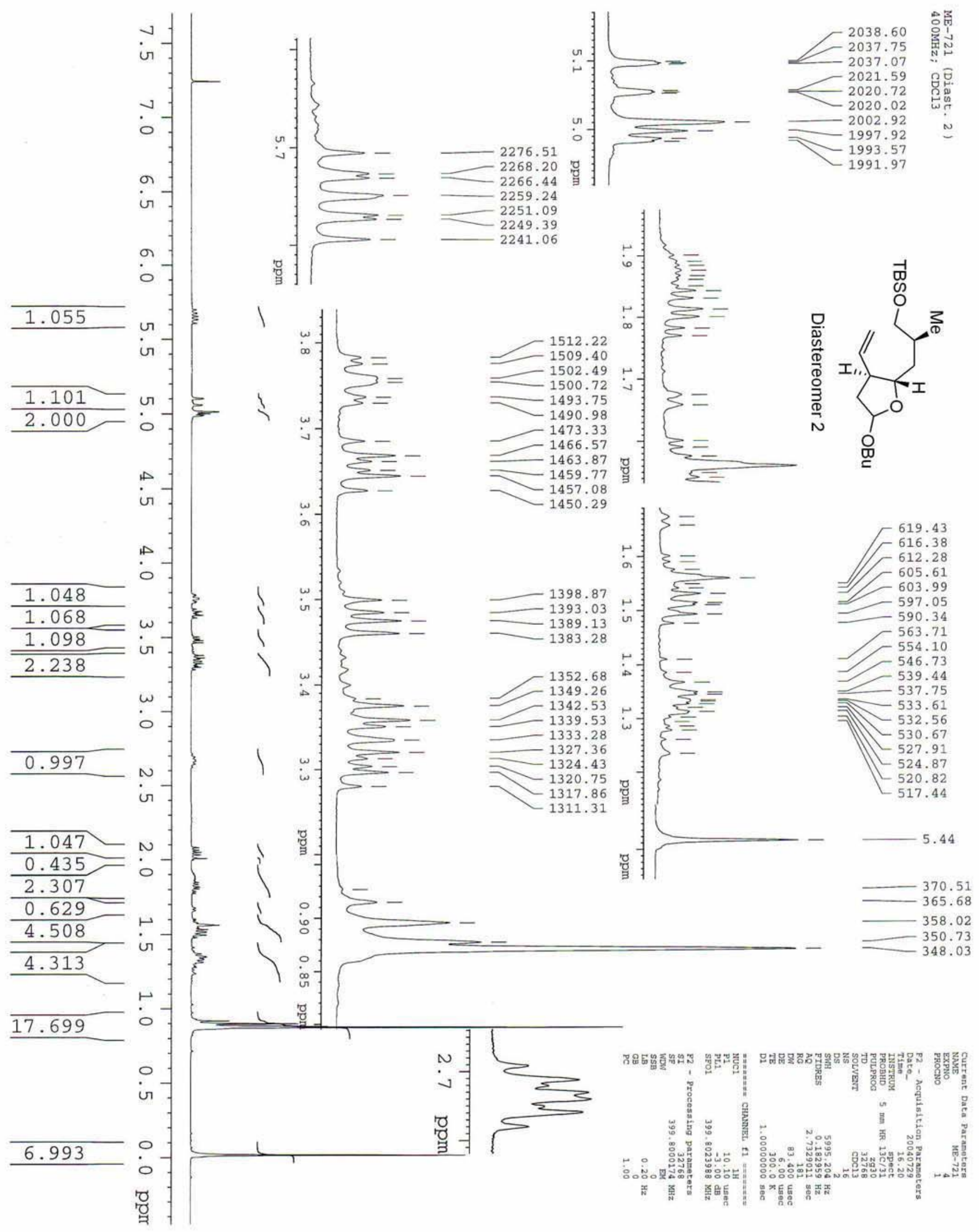




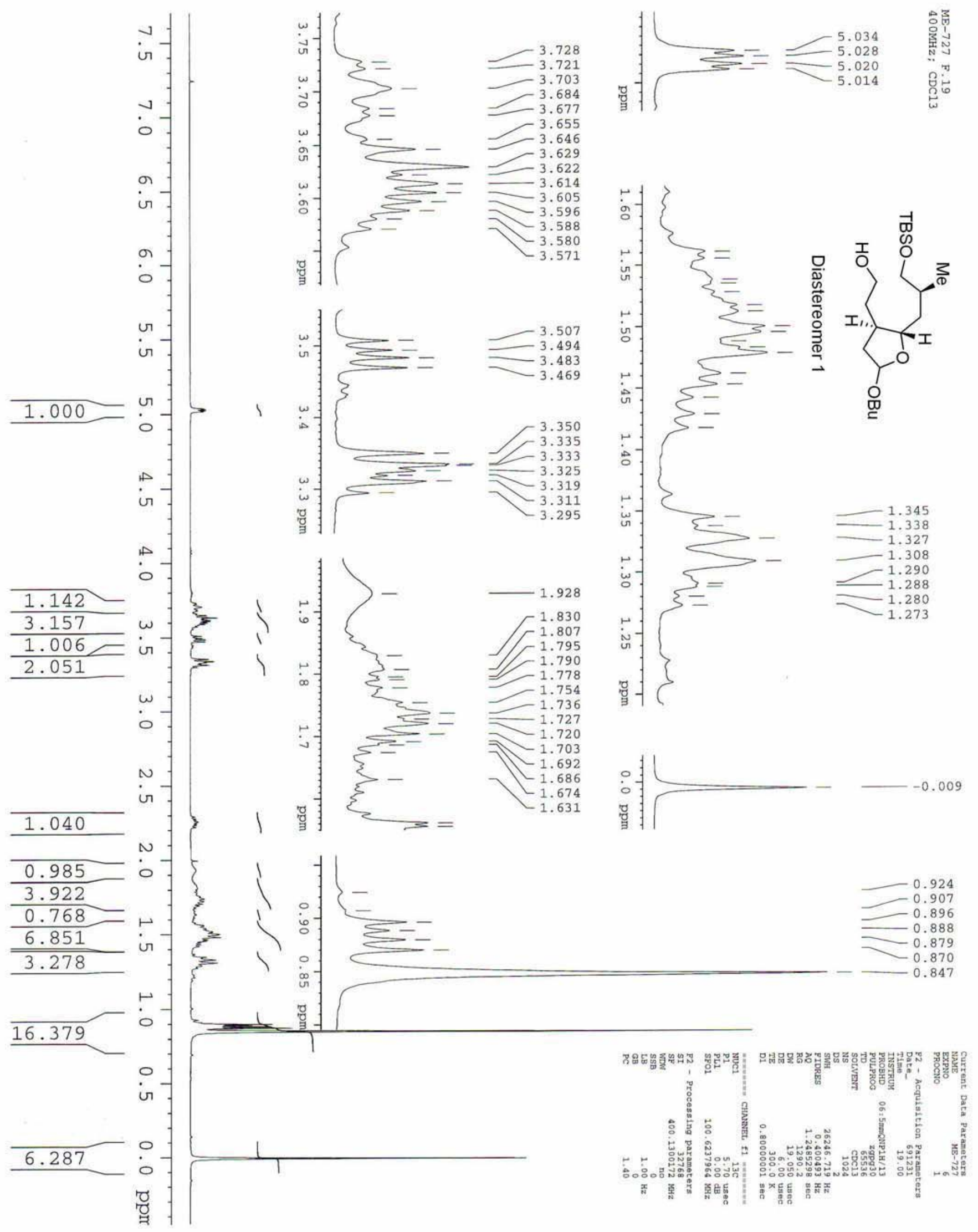



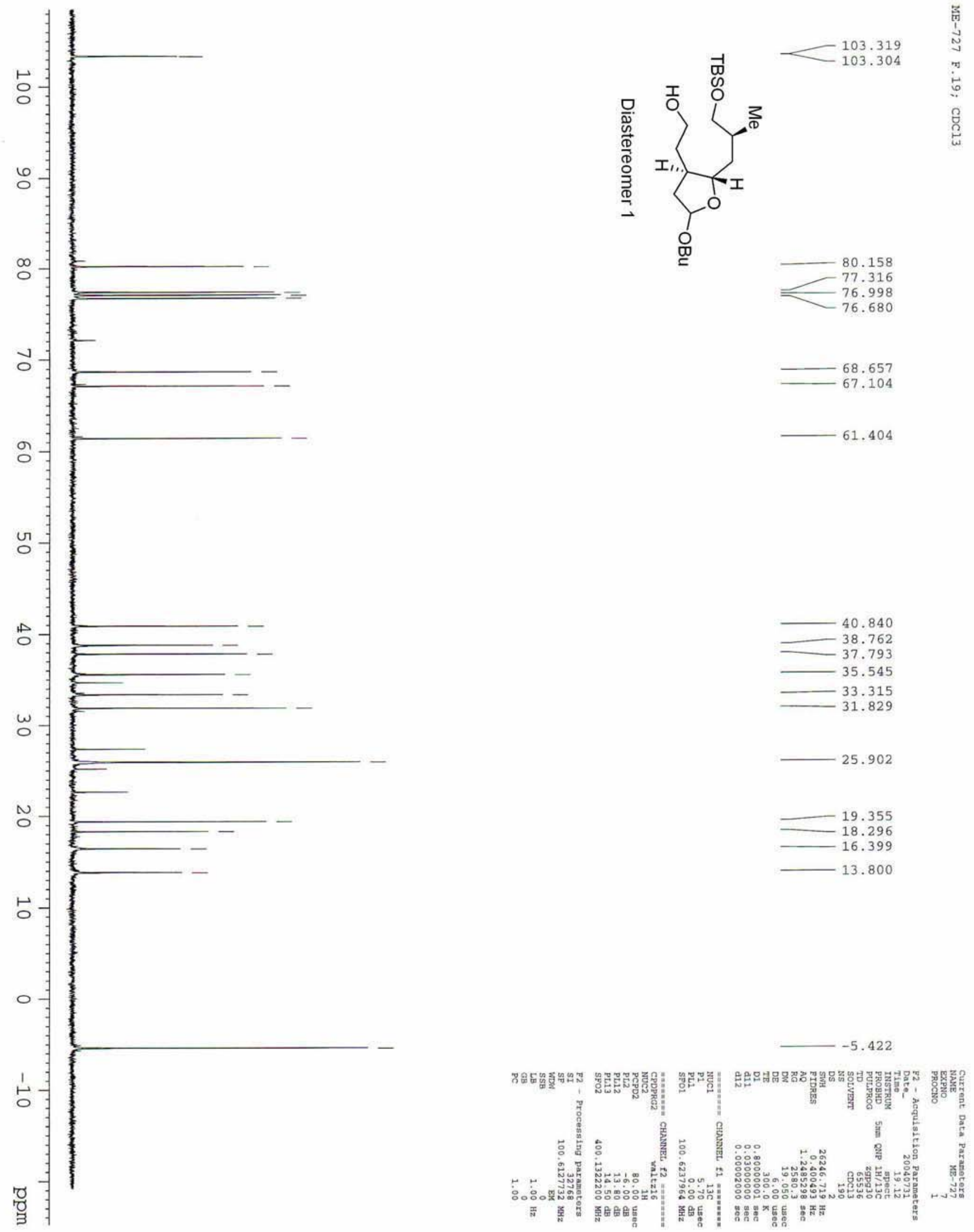

Page S 23 


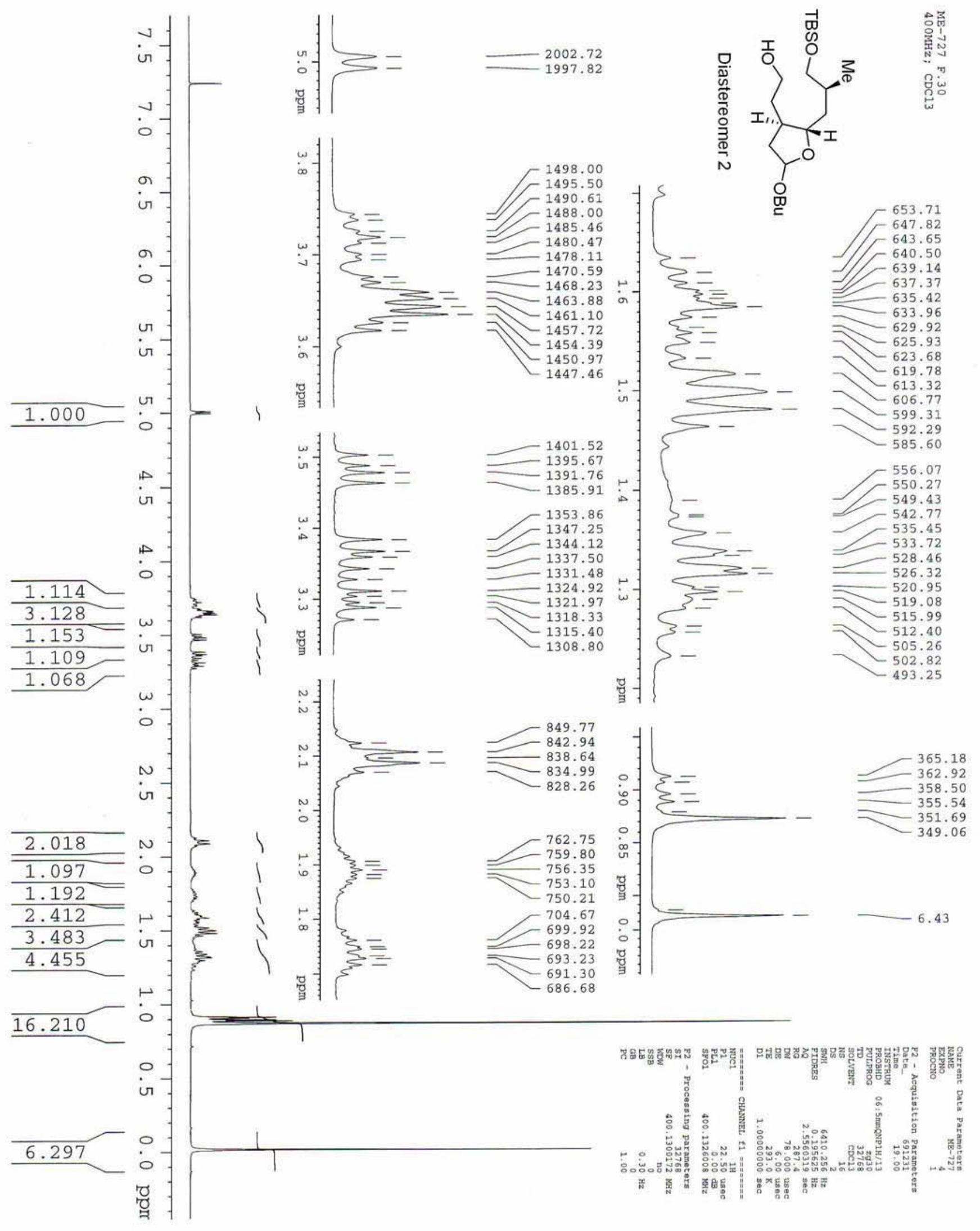



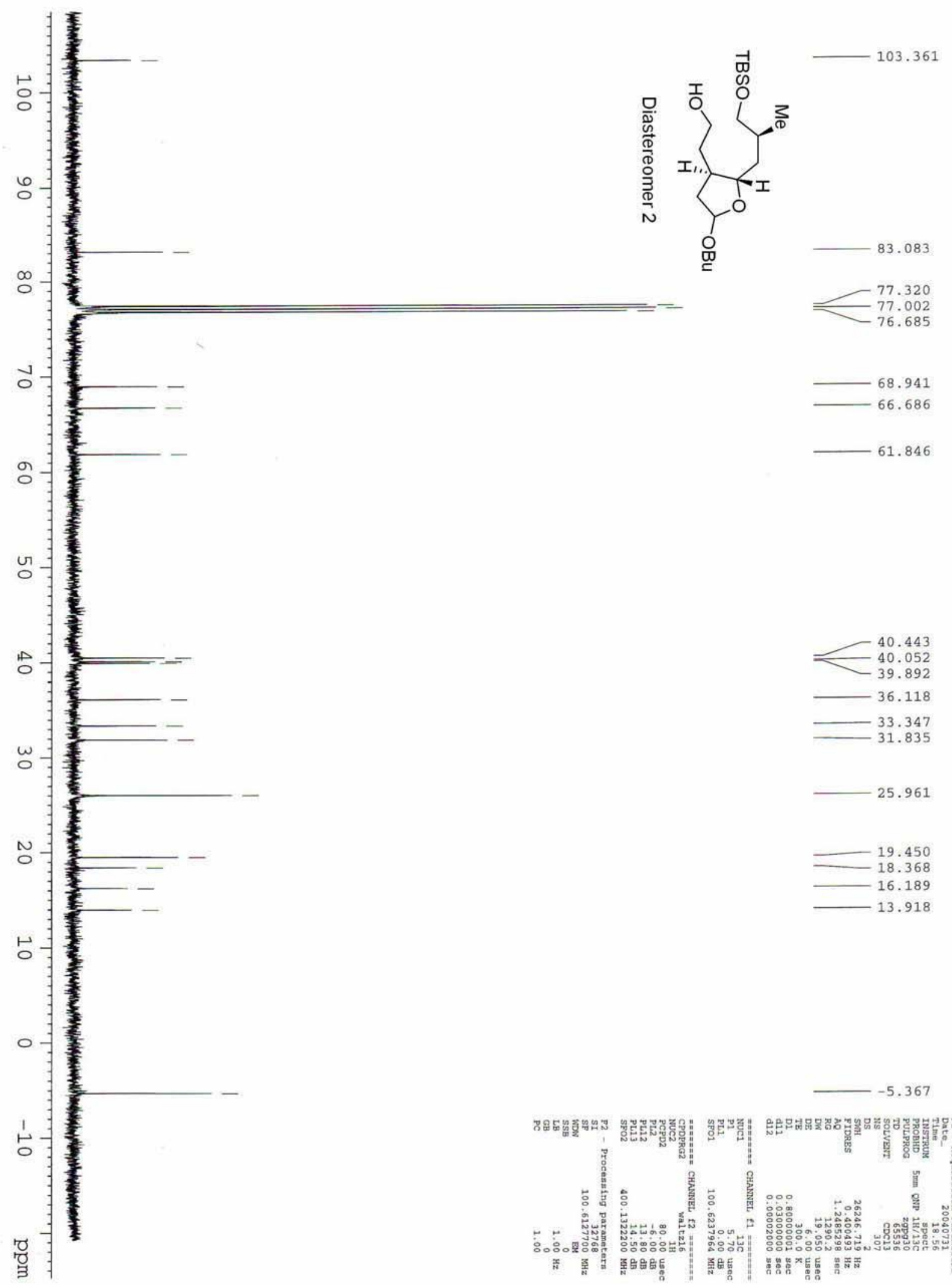

61.846
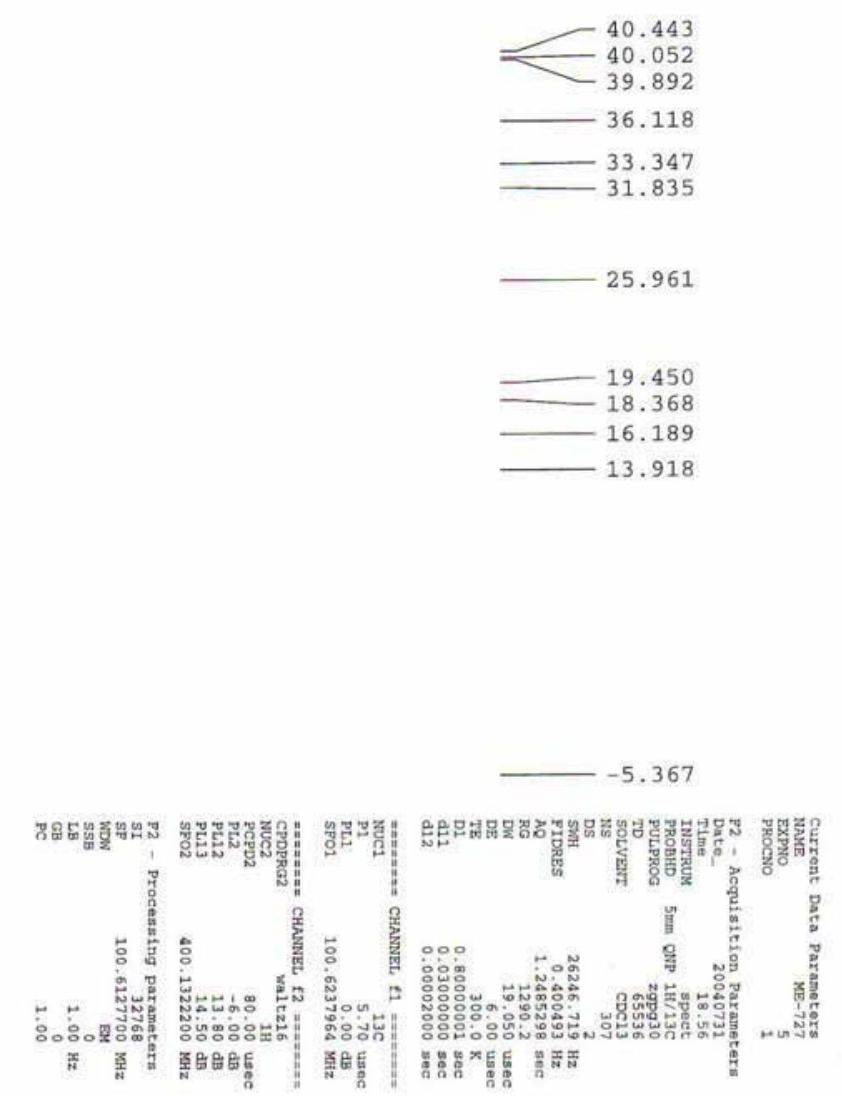

Page S 25 


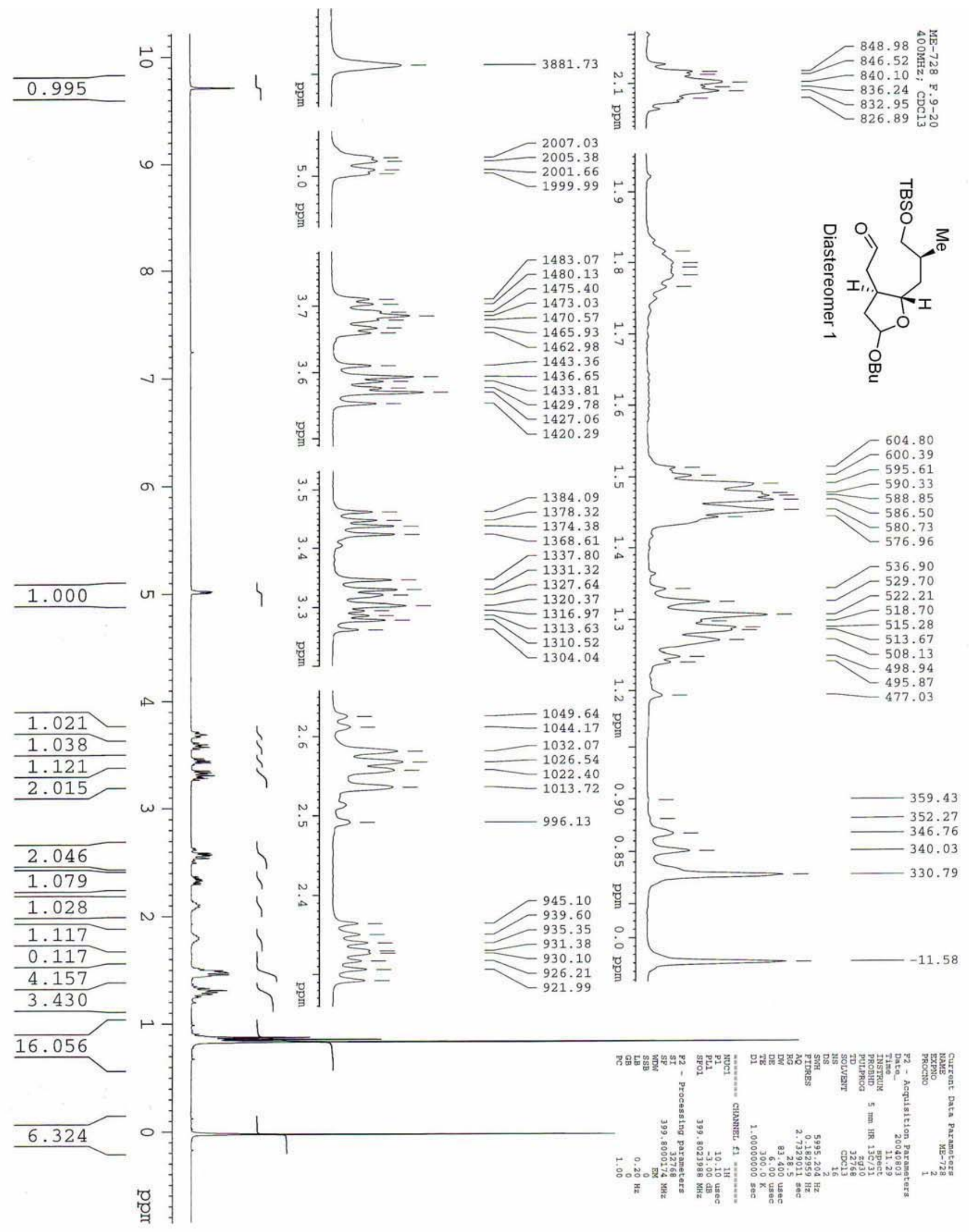




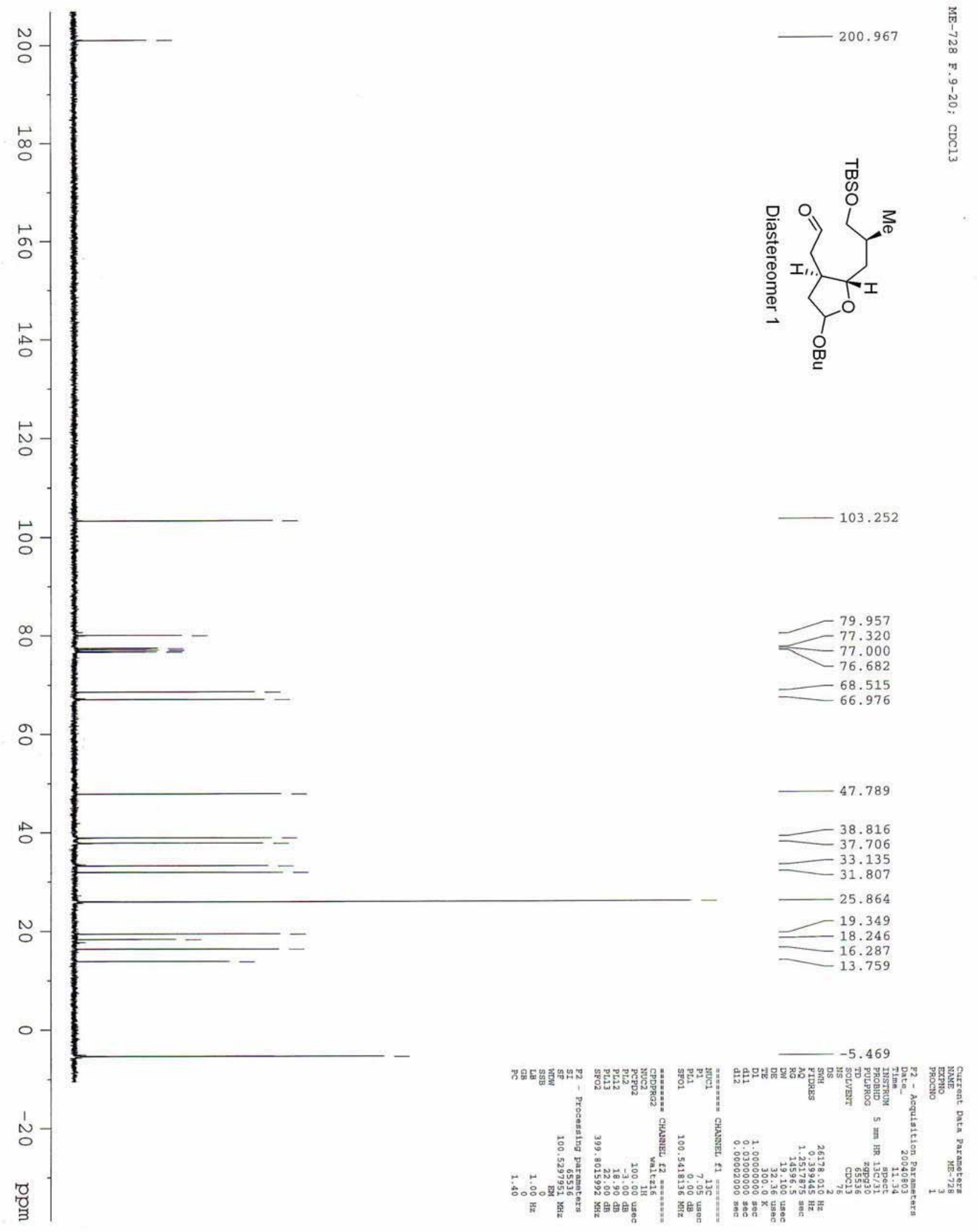

Page S 27 


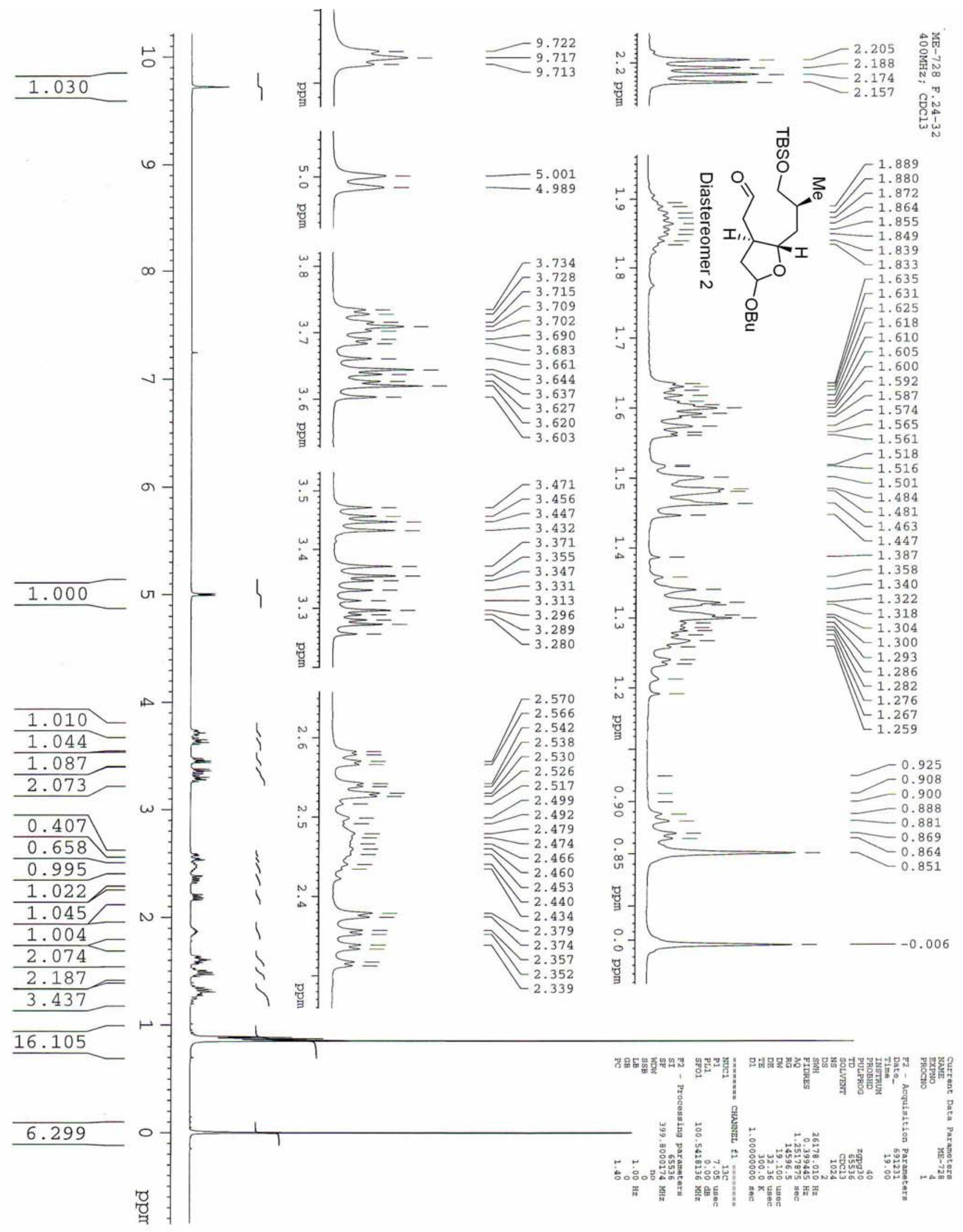




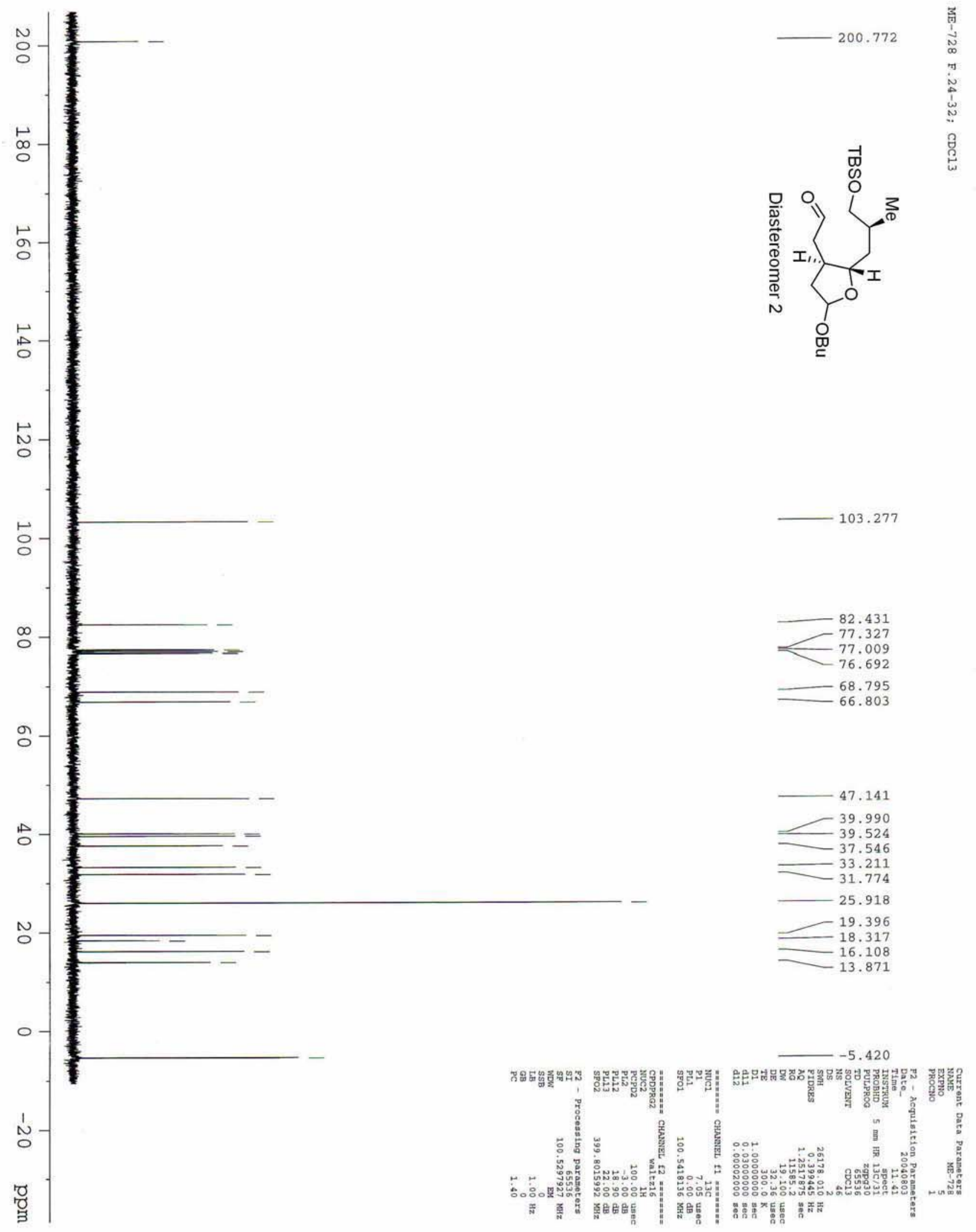

Page S 29 


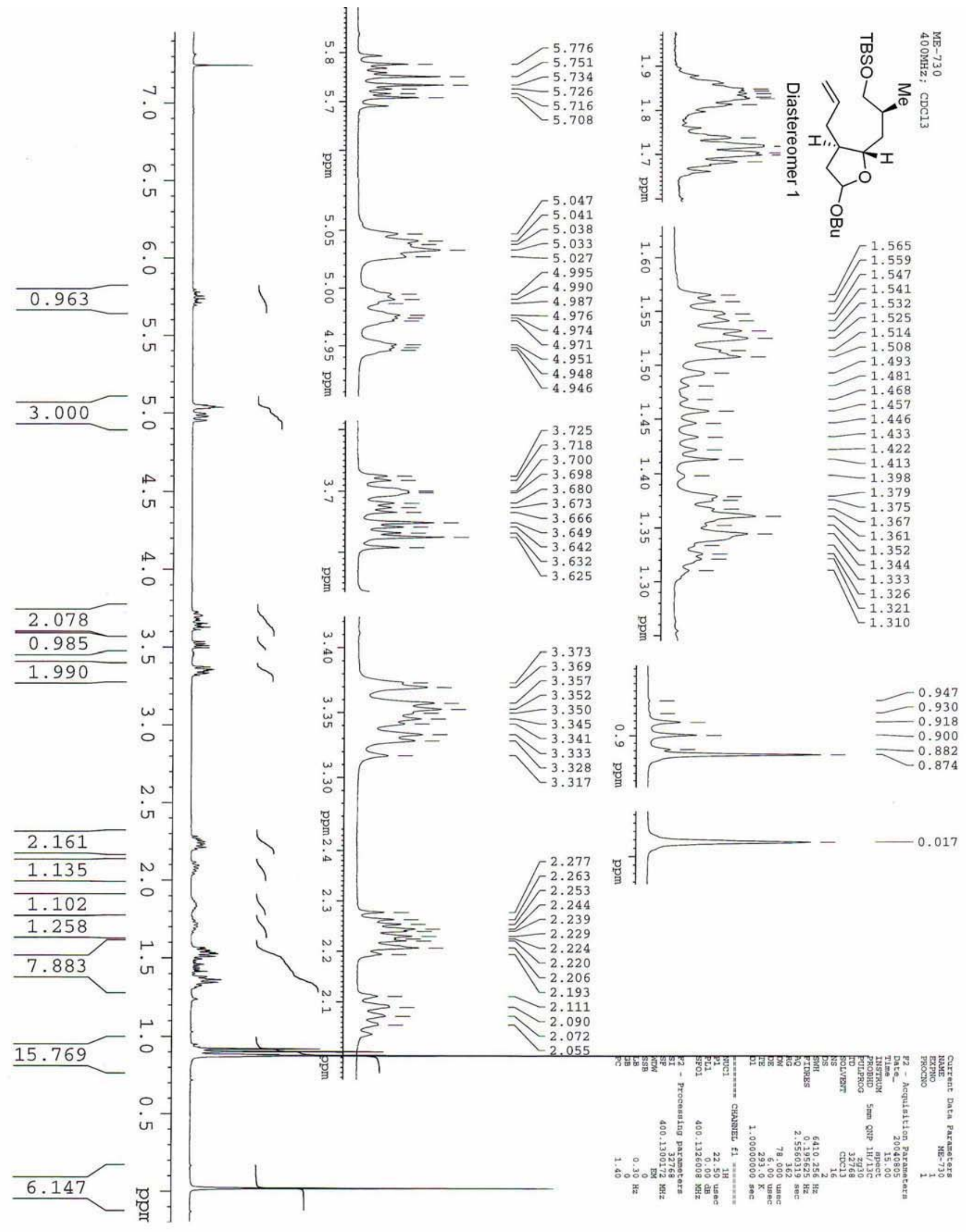




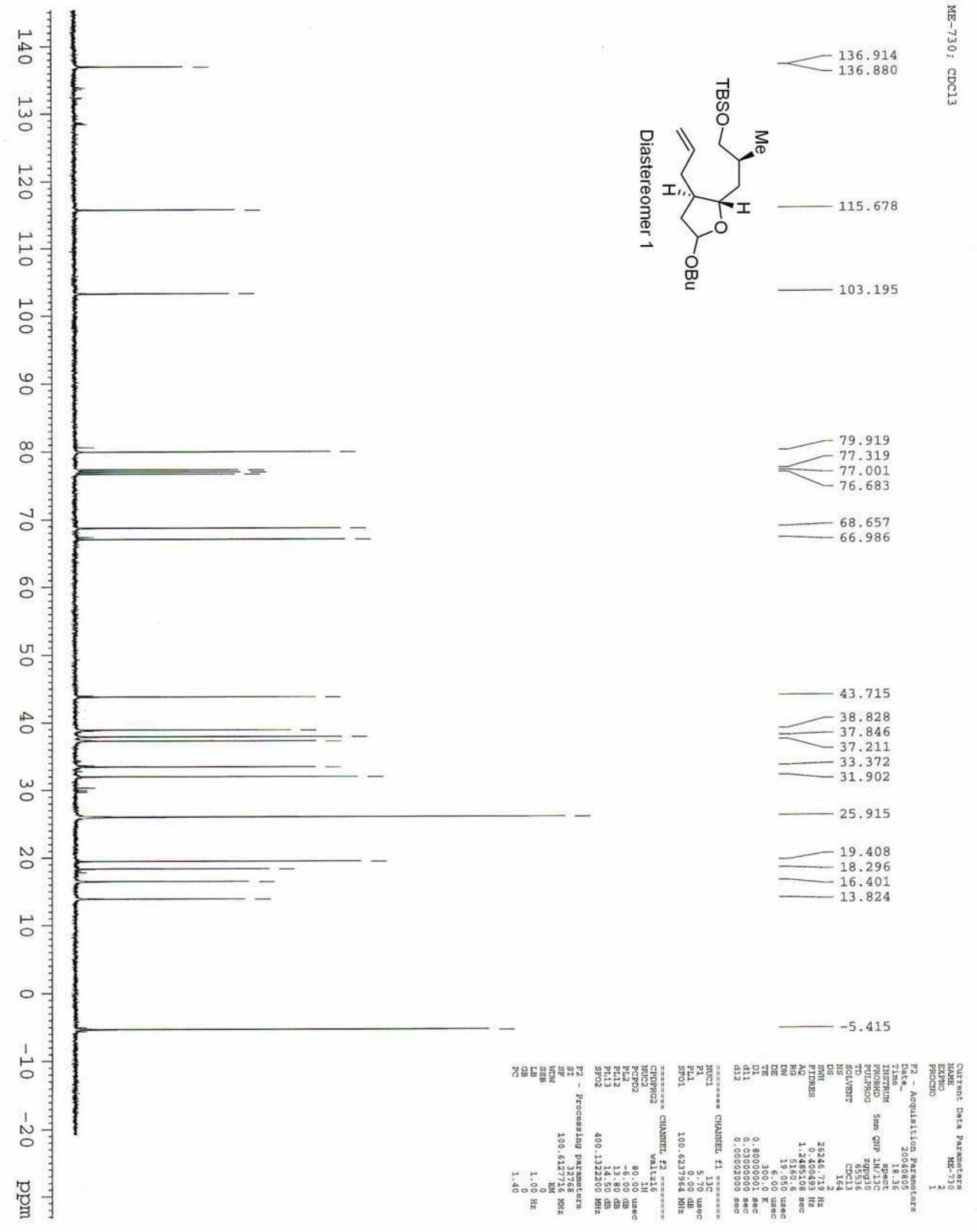

Page S 31 


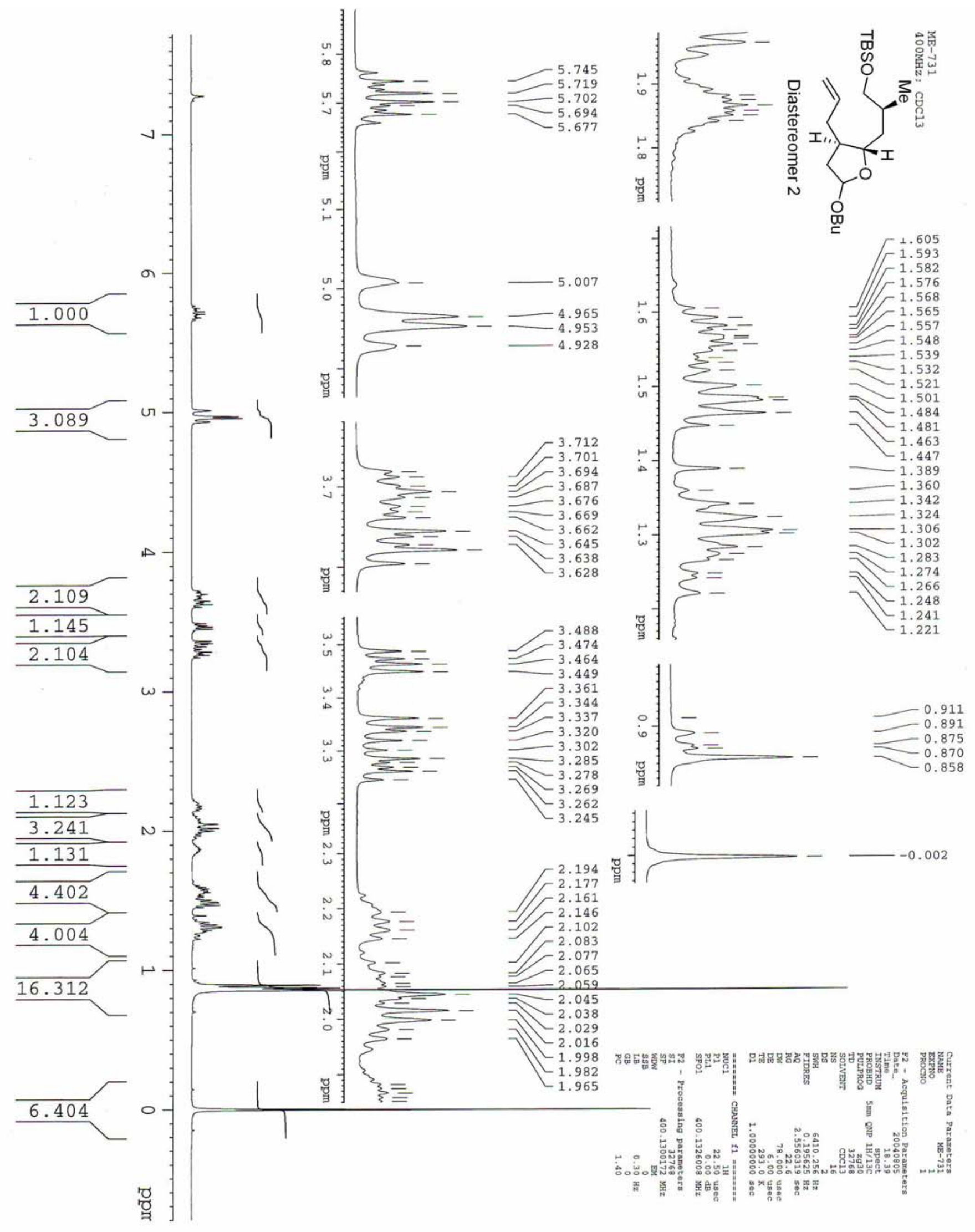




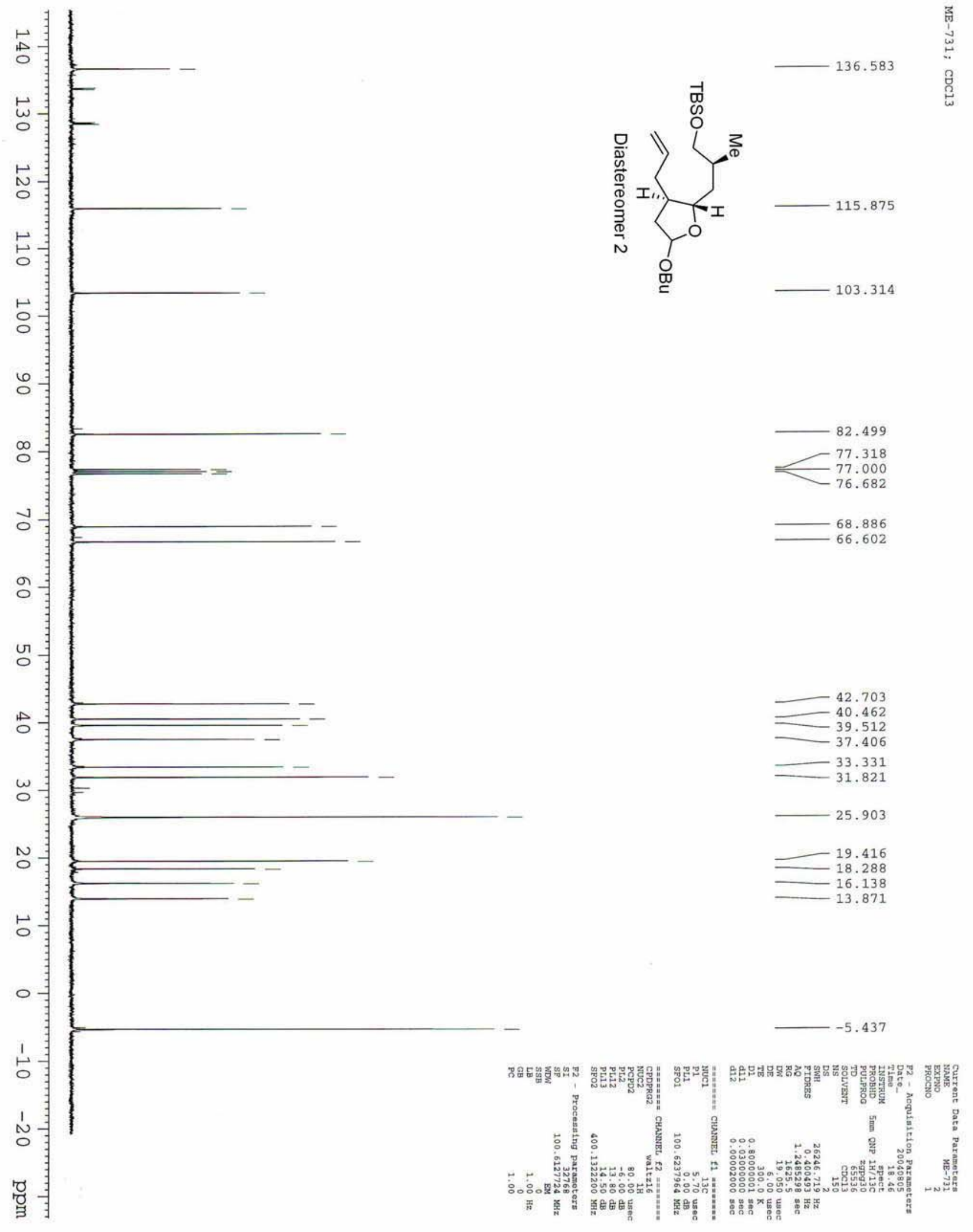

Page S 33 


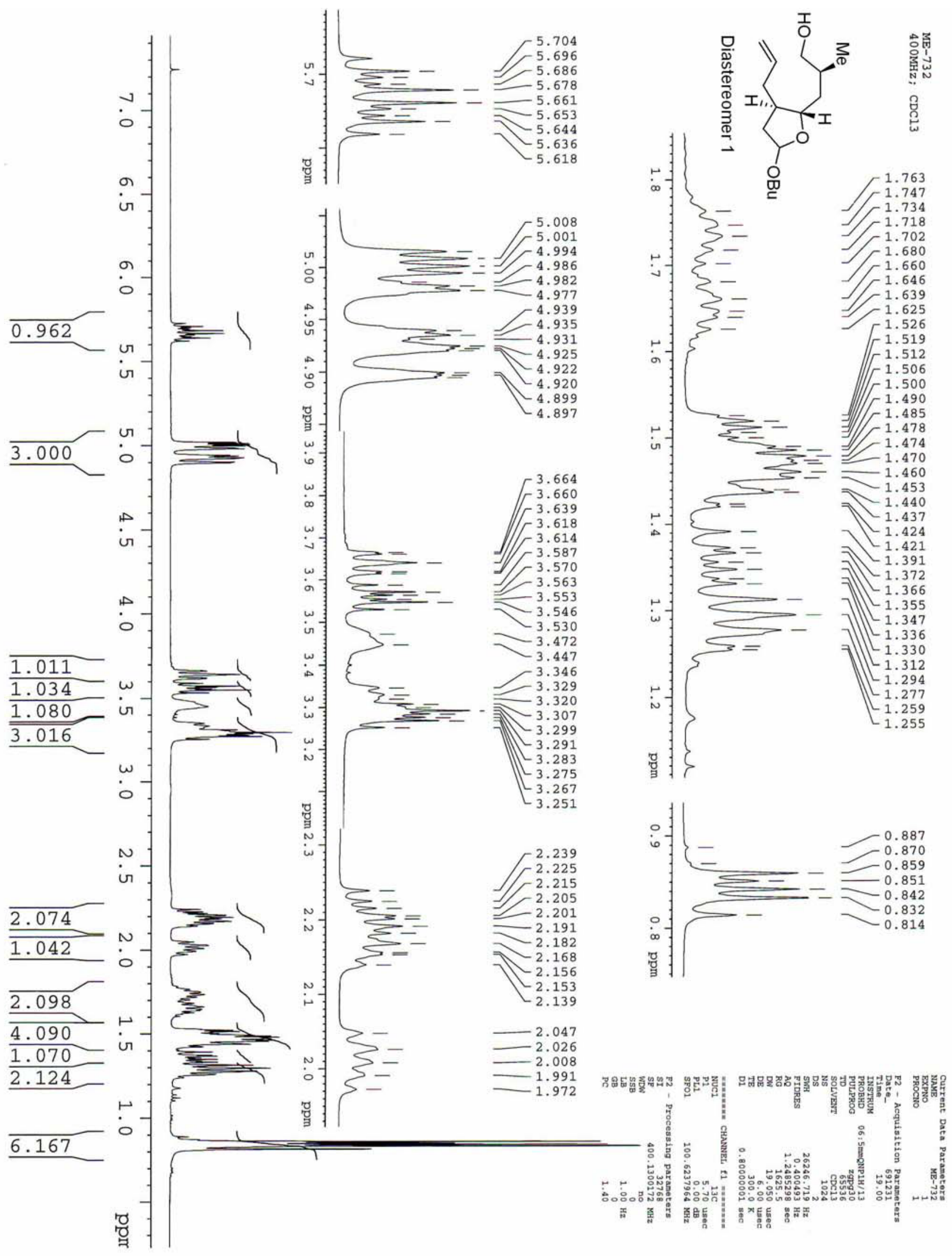




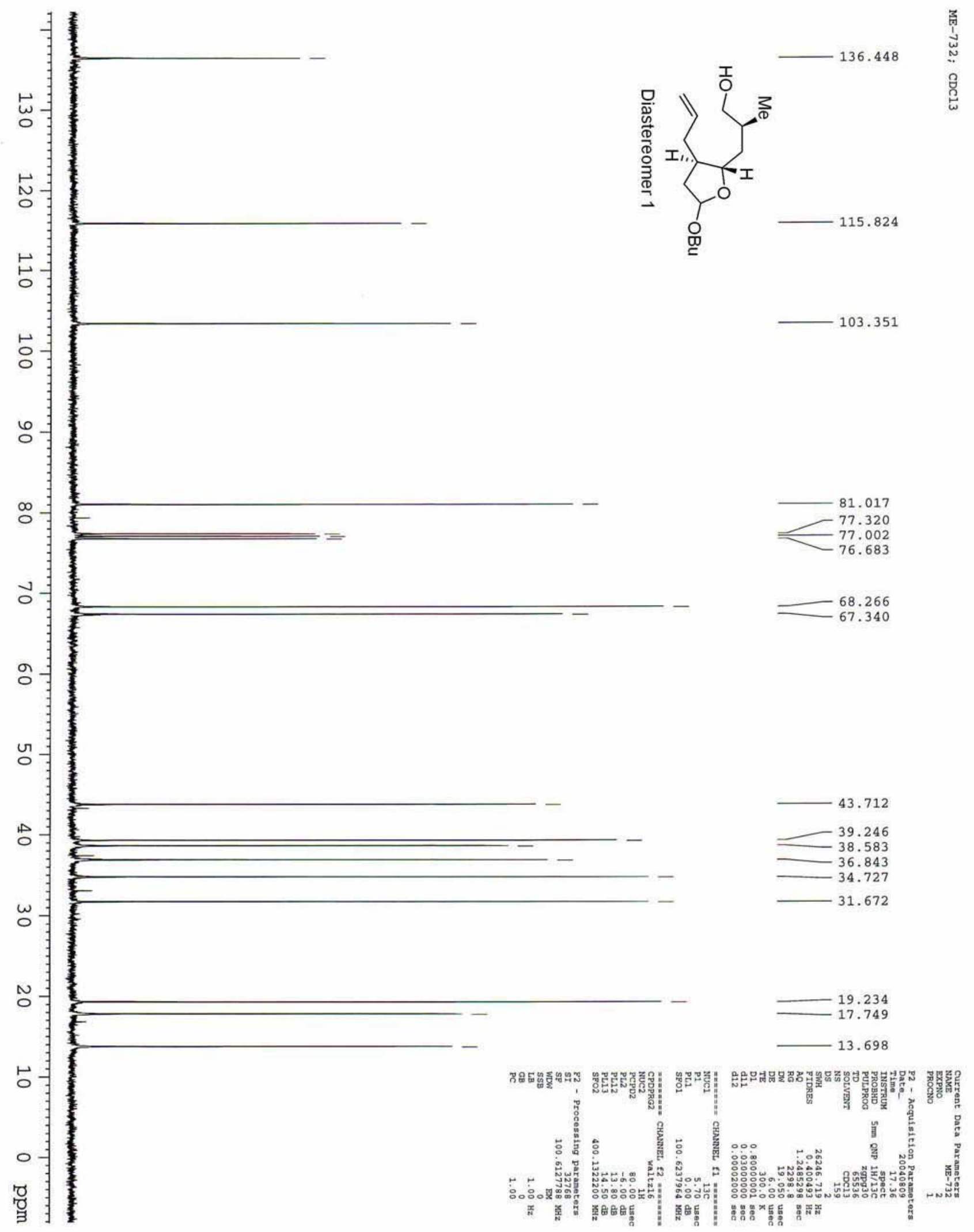

Page S 35 


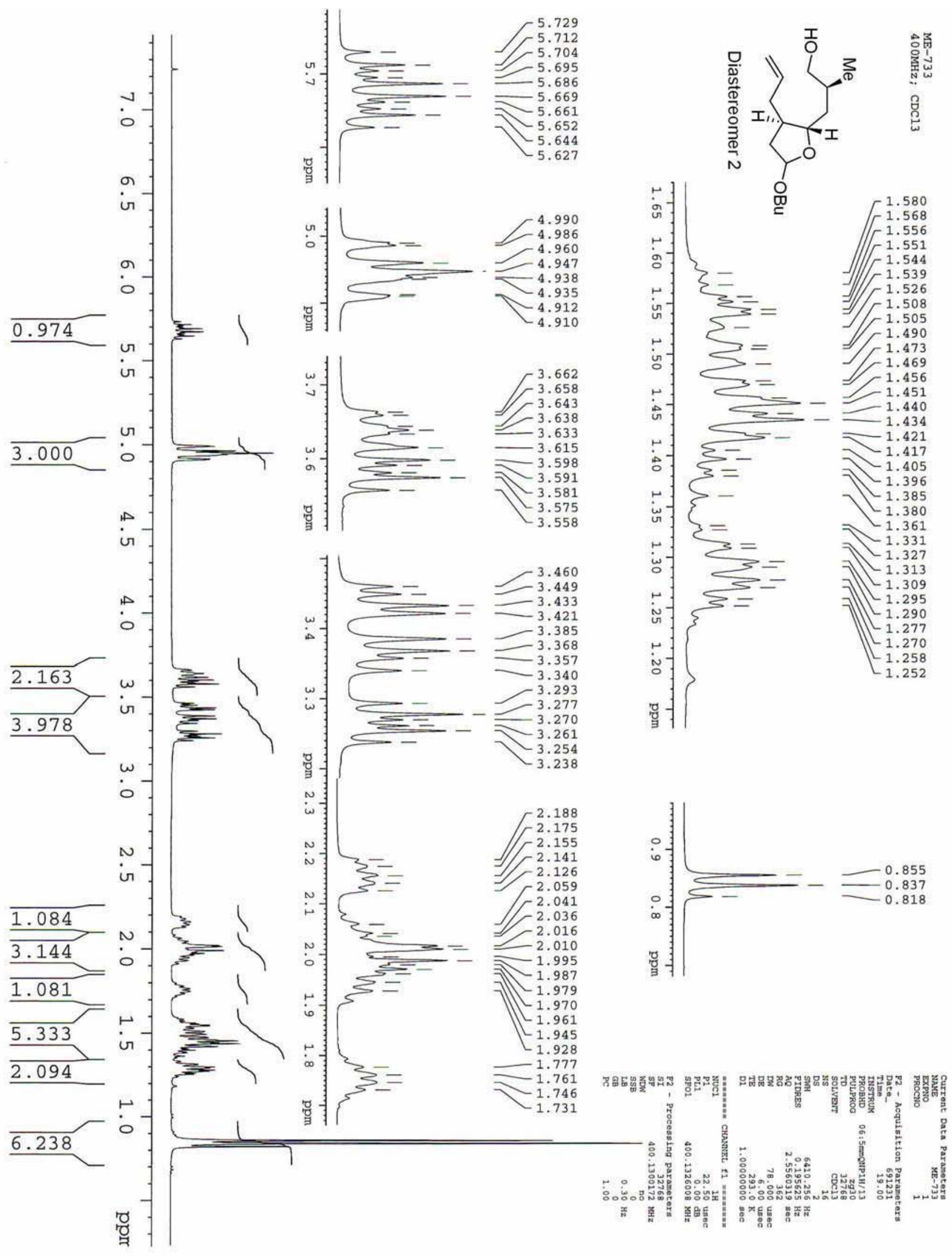




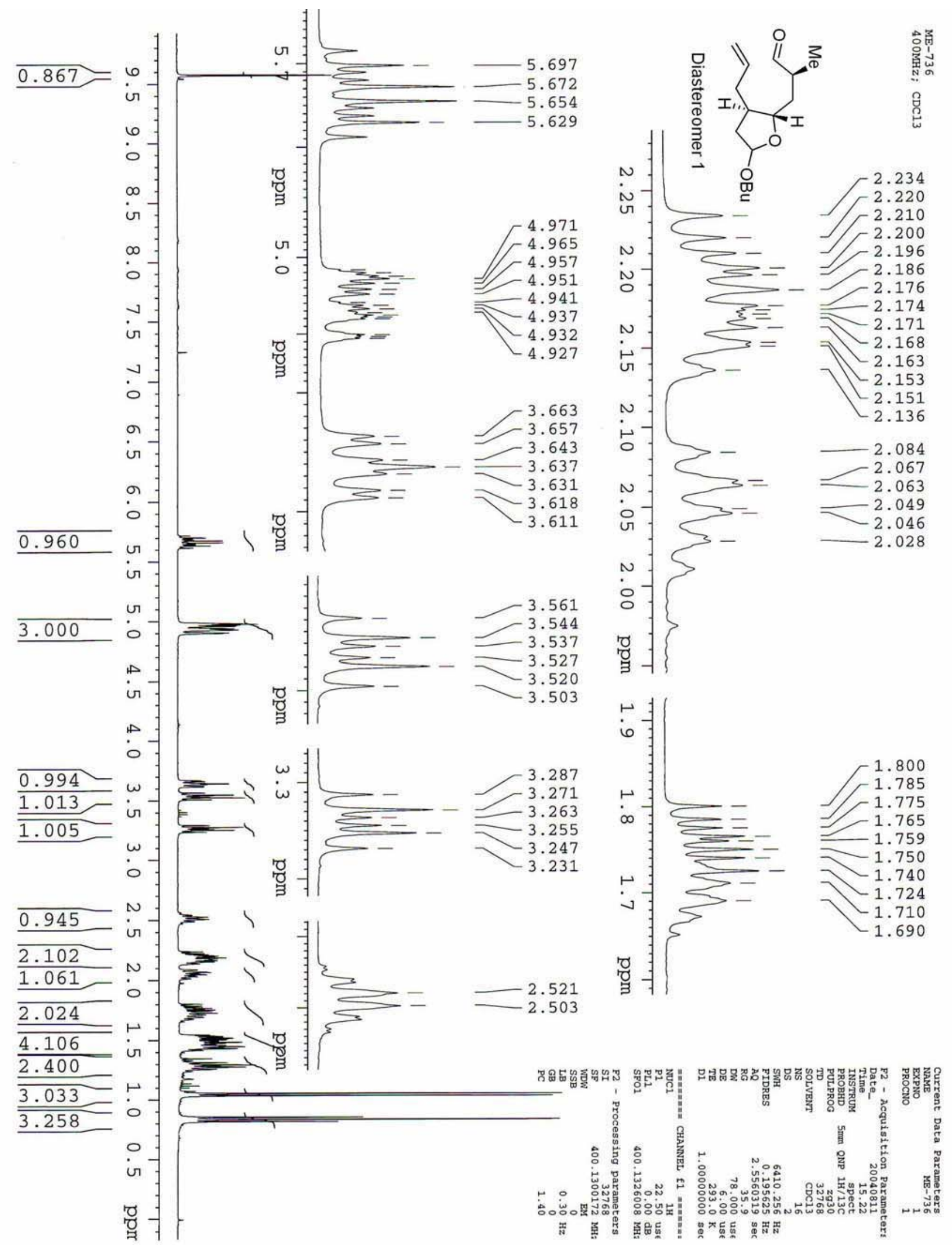




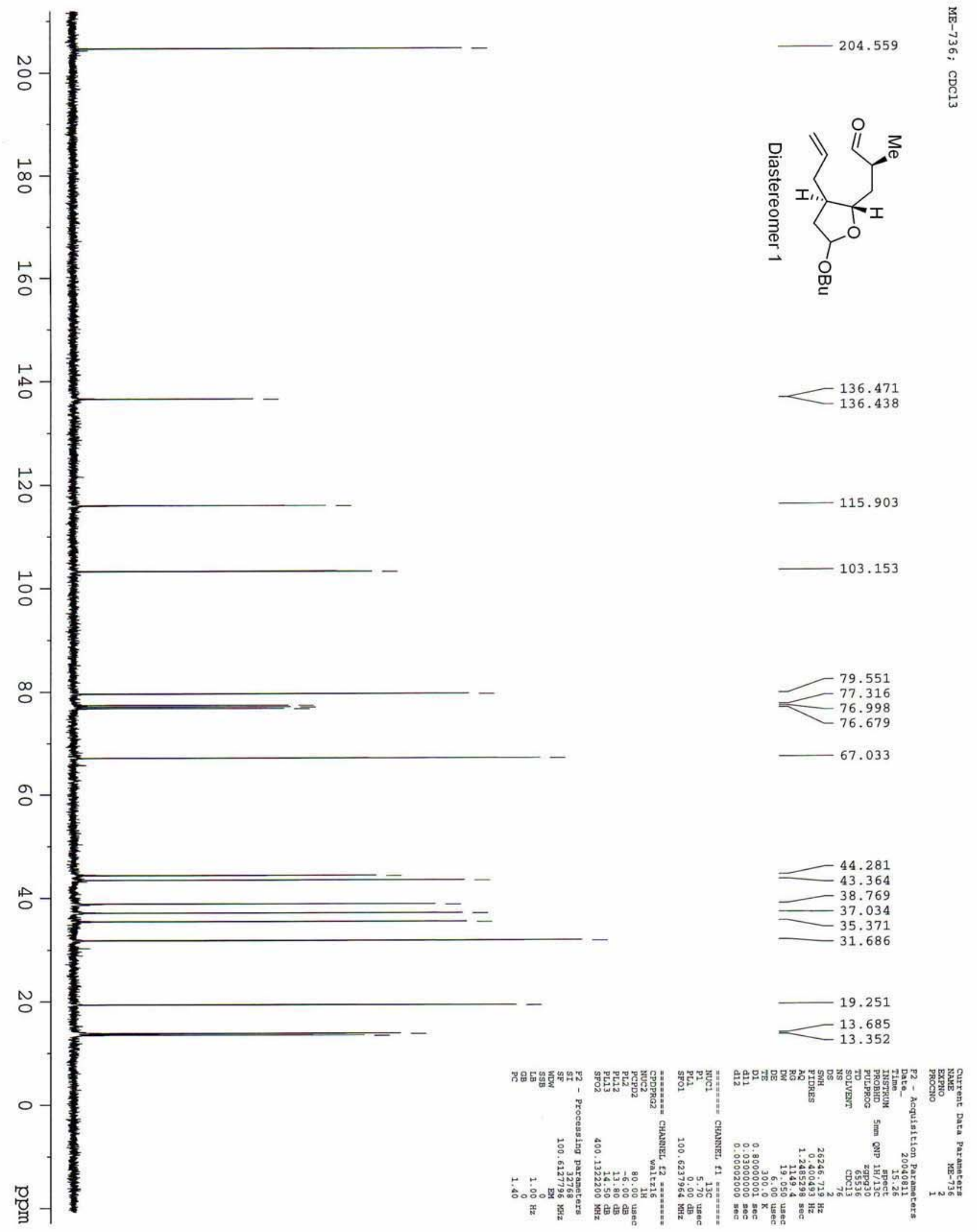

Page S 38 


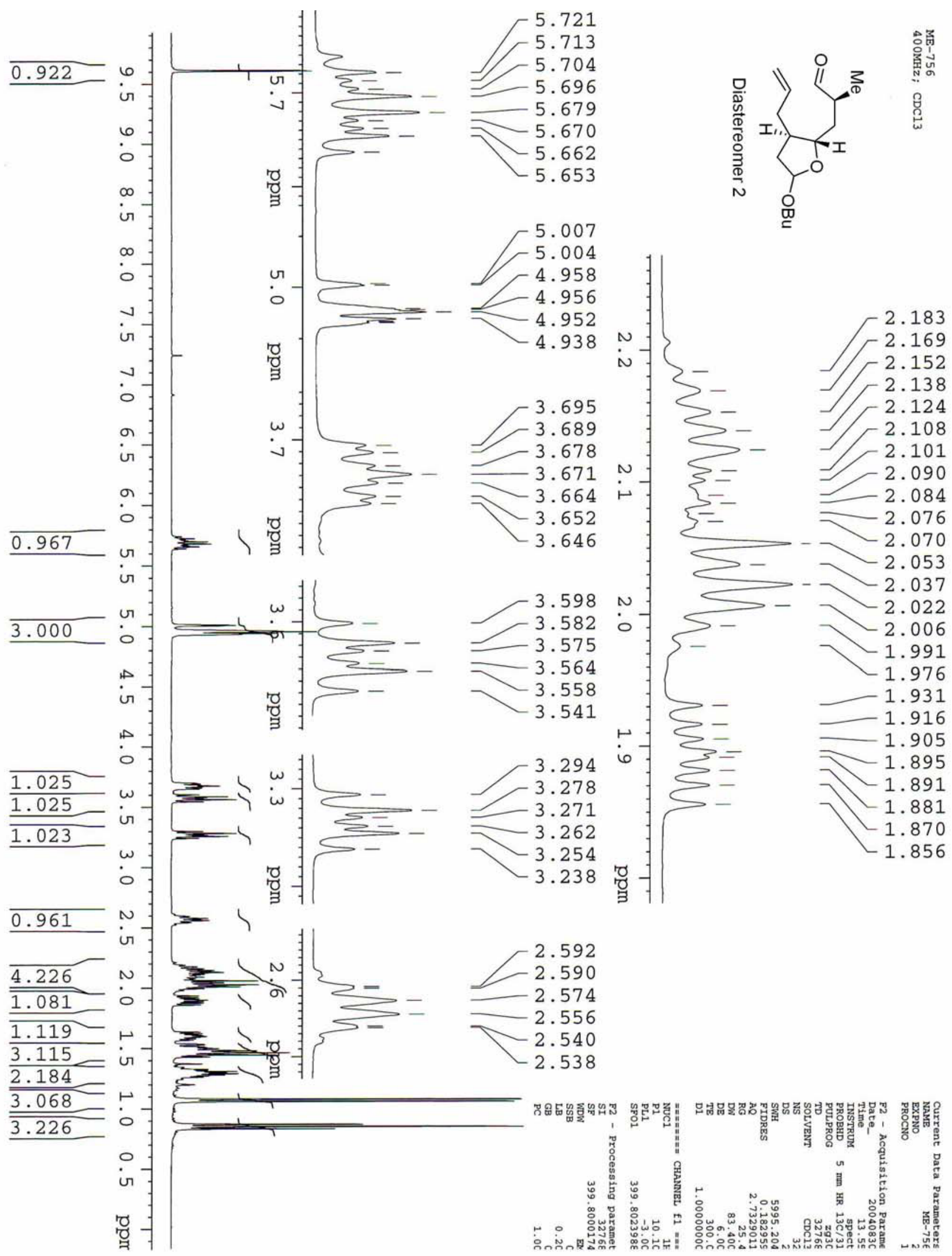




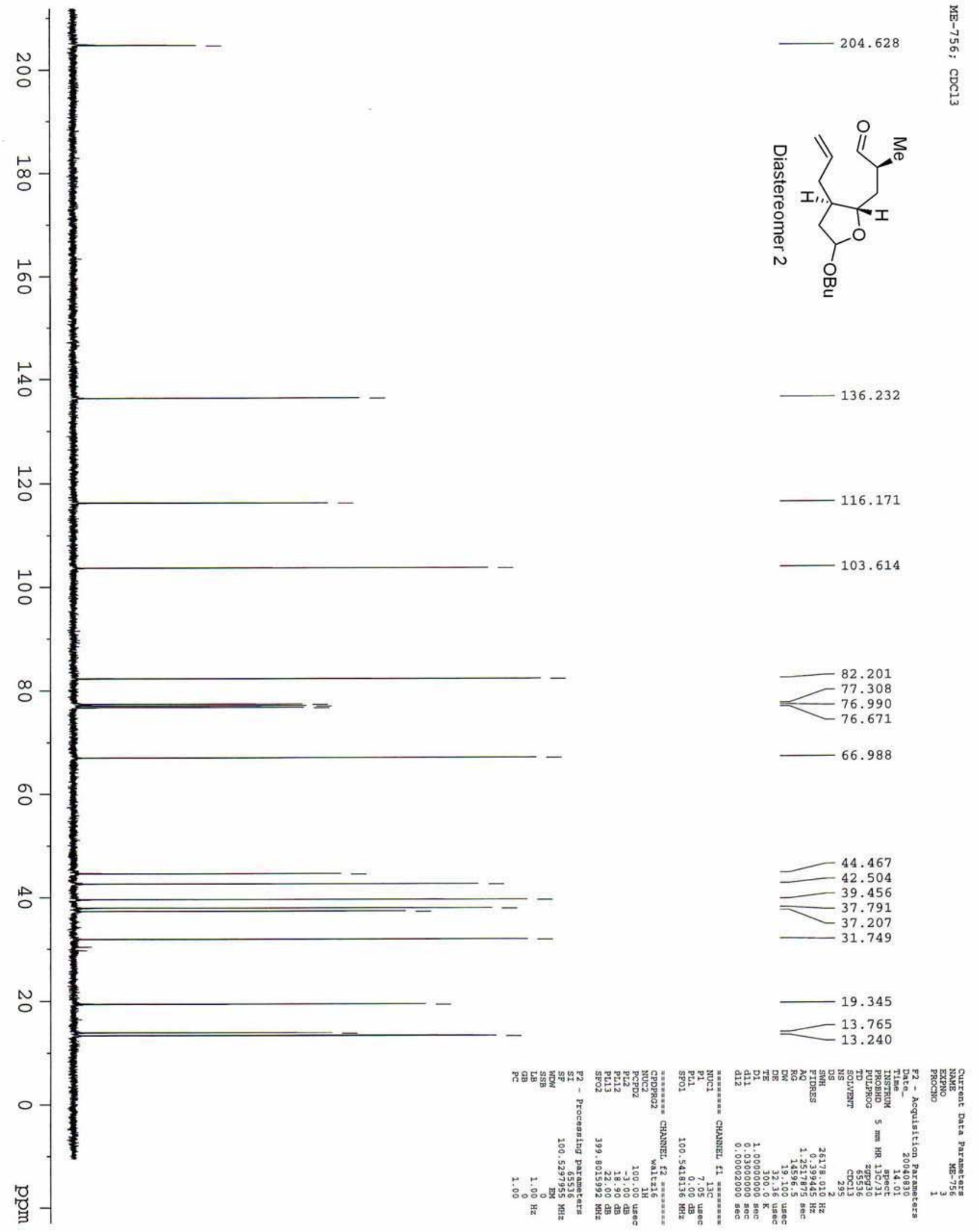

Page S 40 


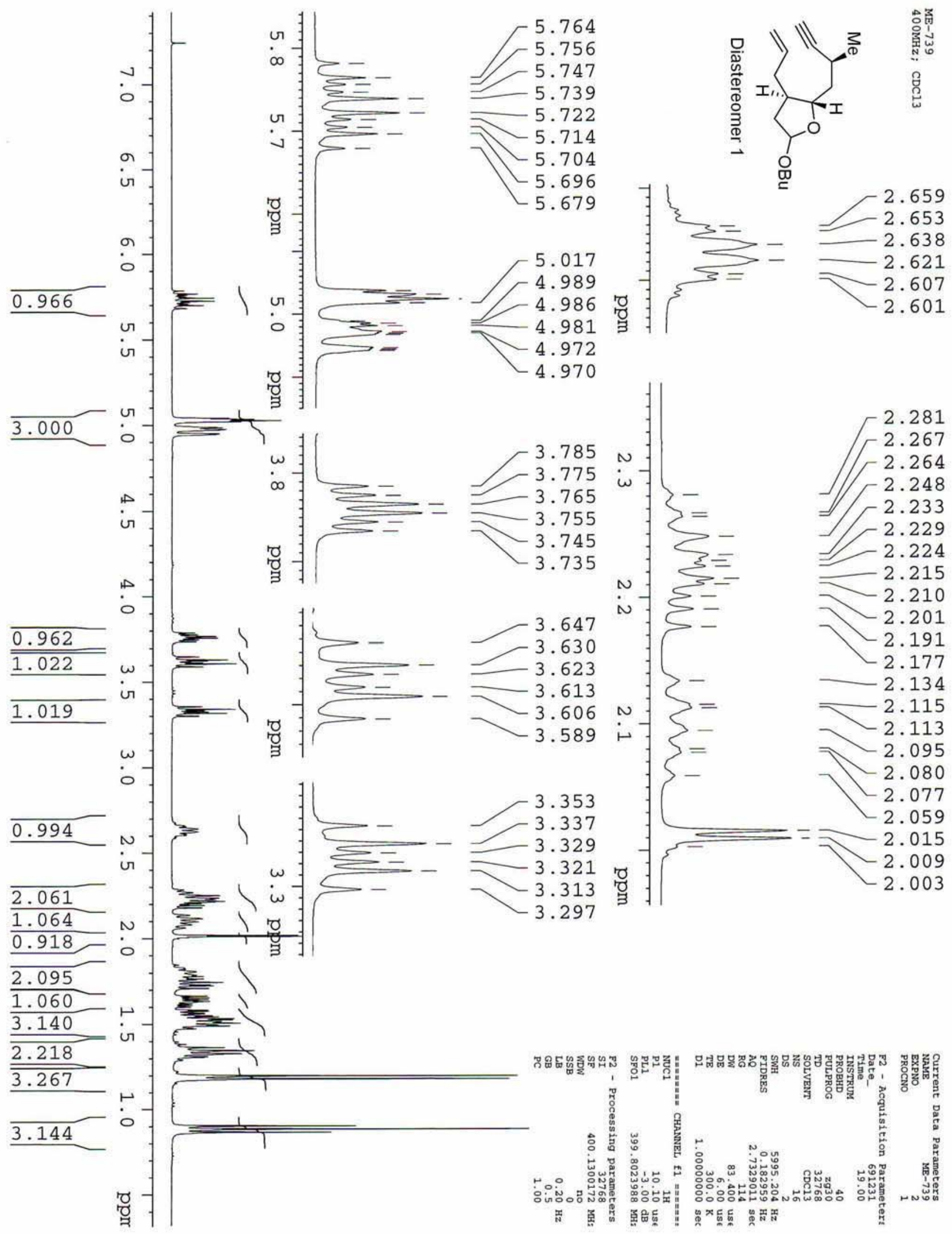




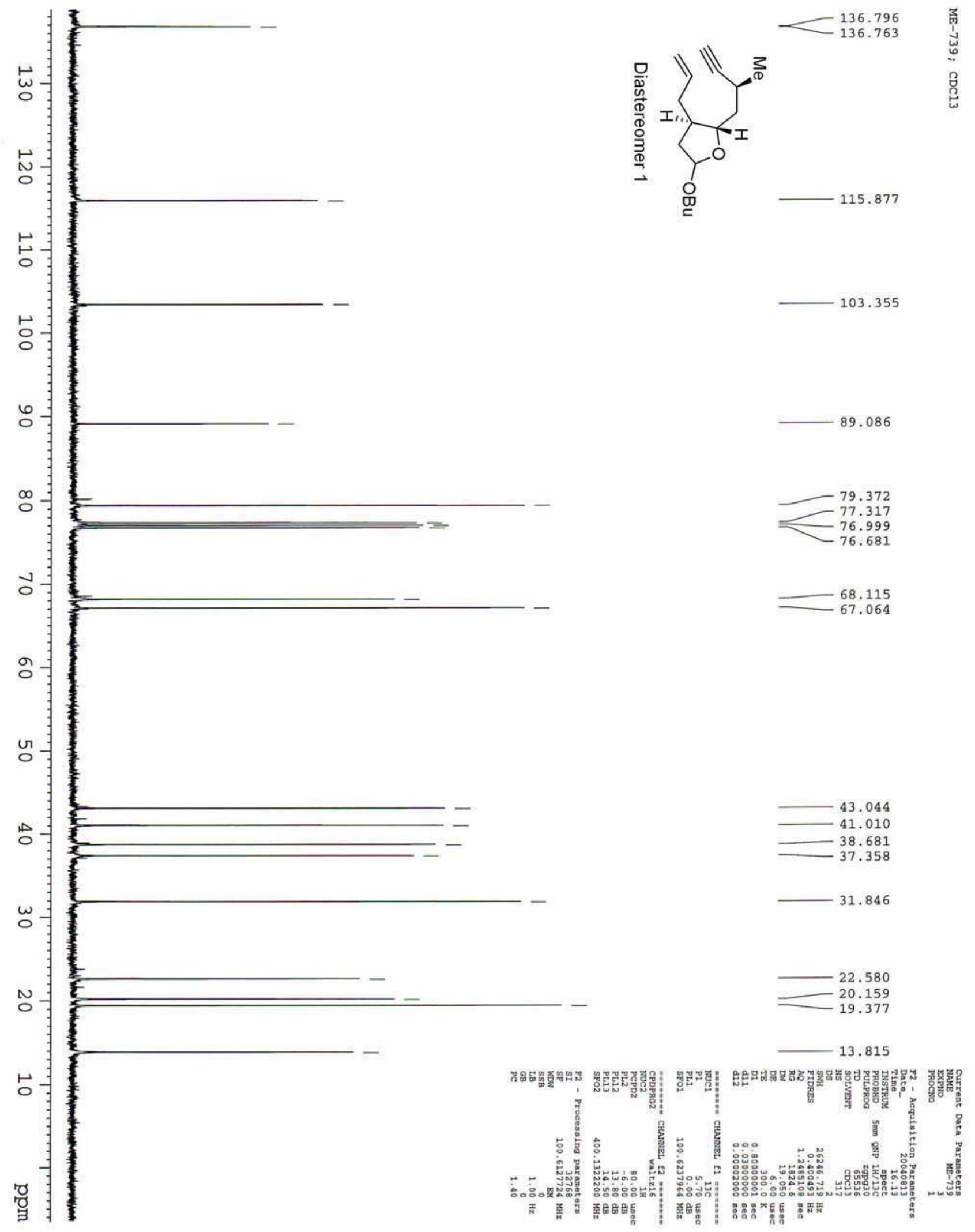

Page S 42 


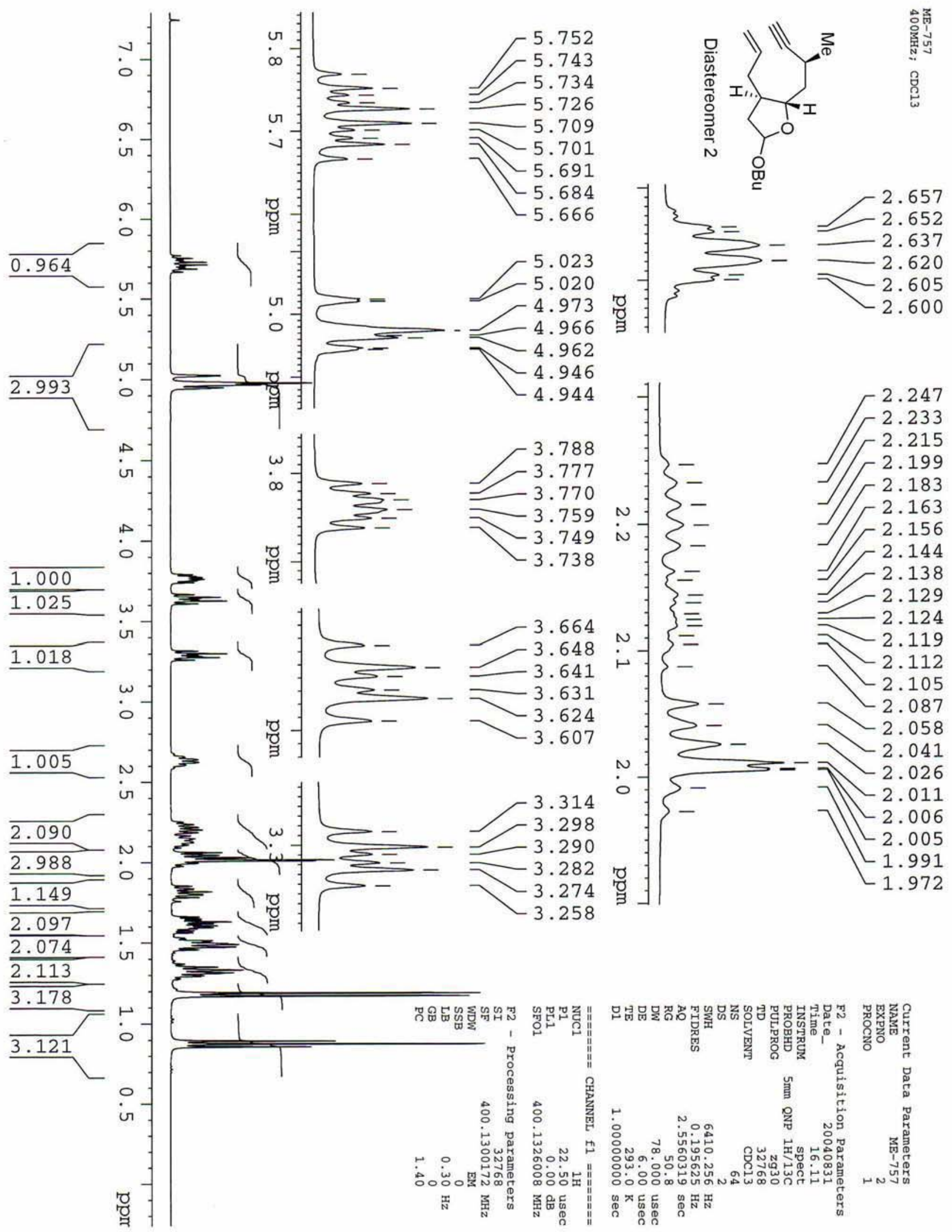




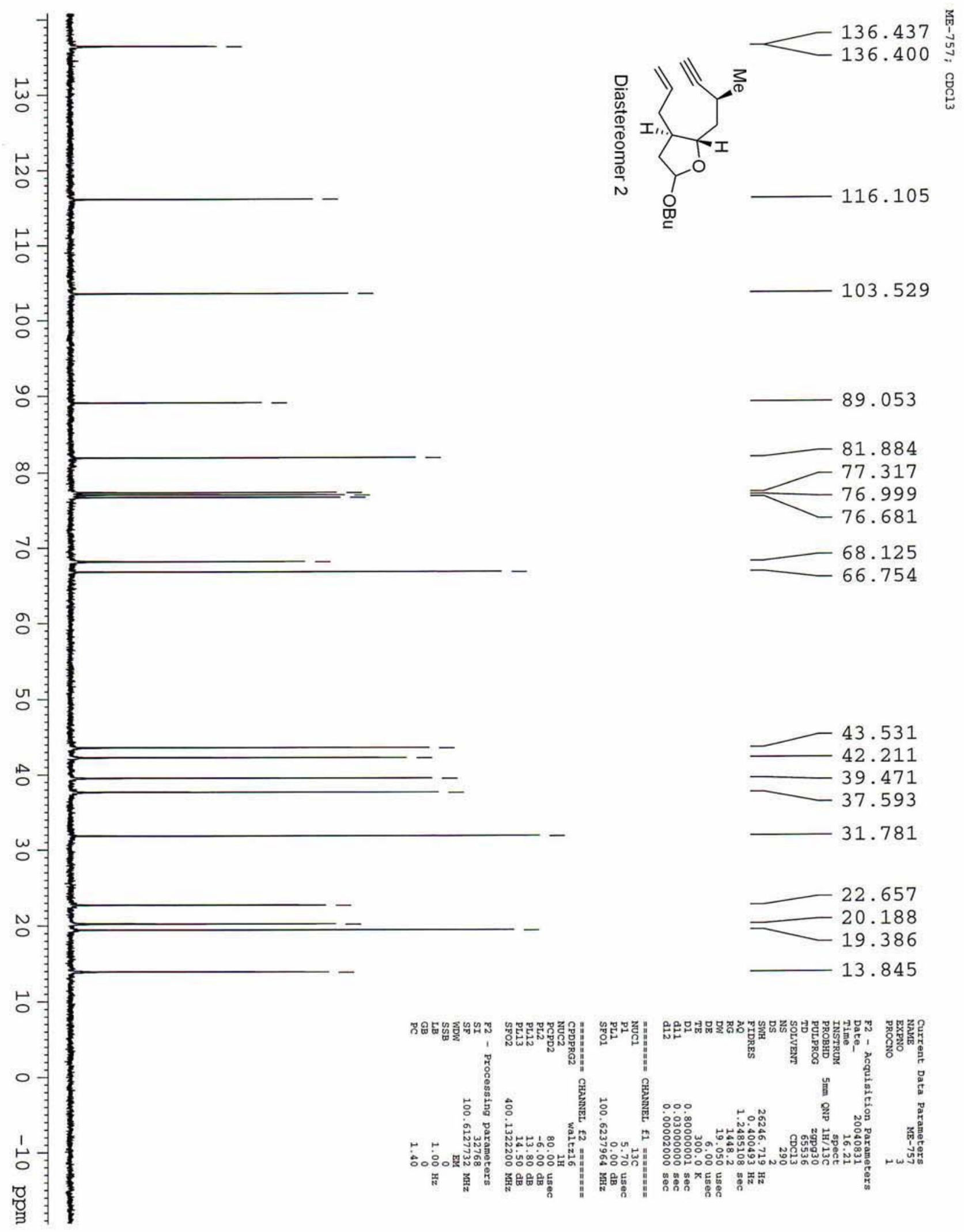




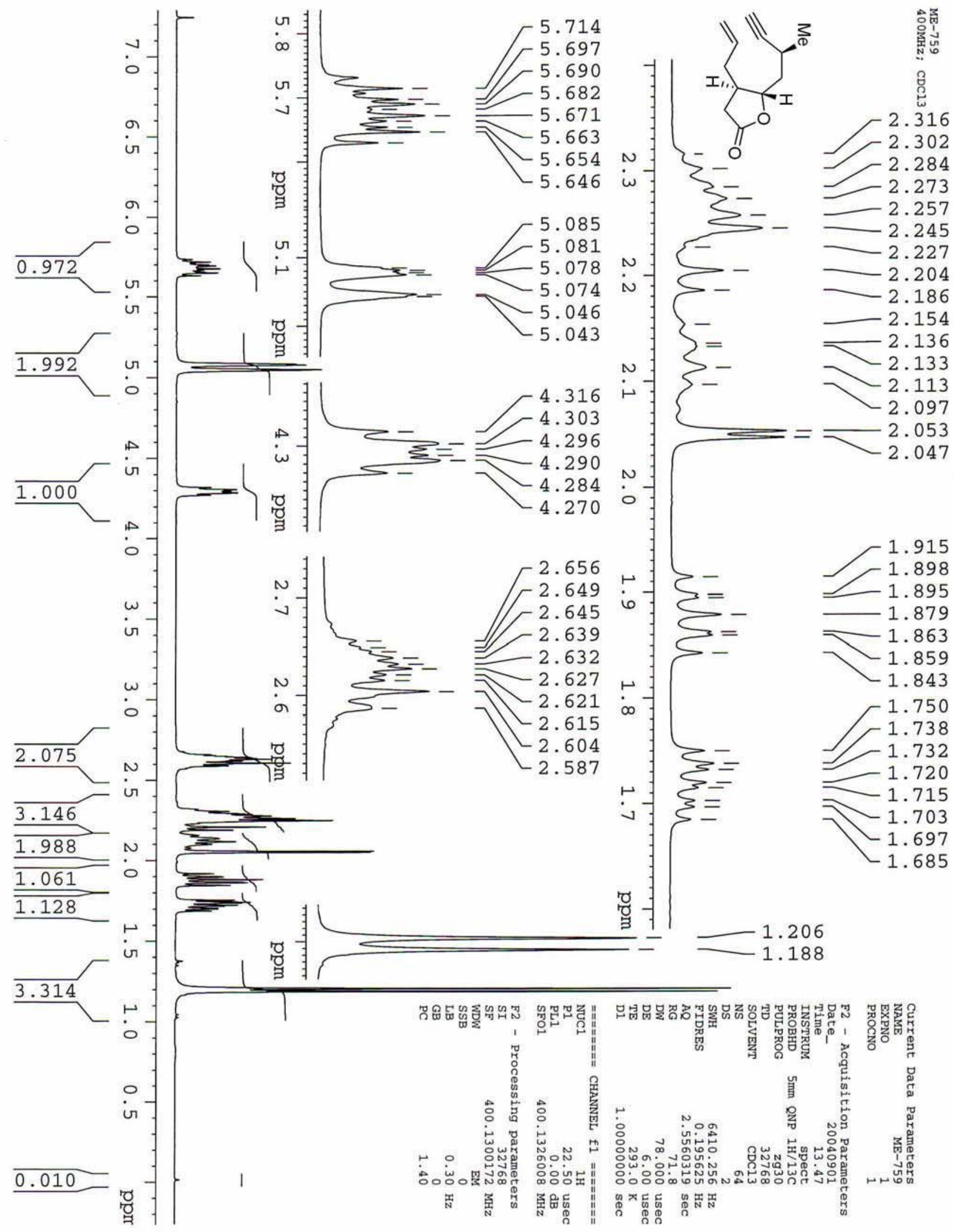




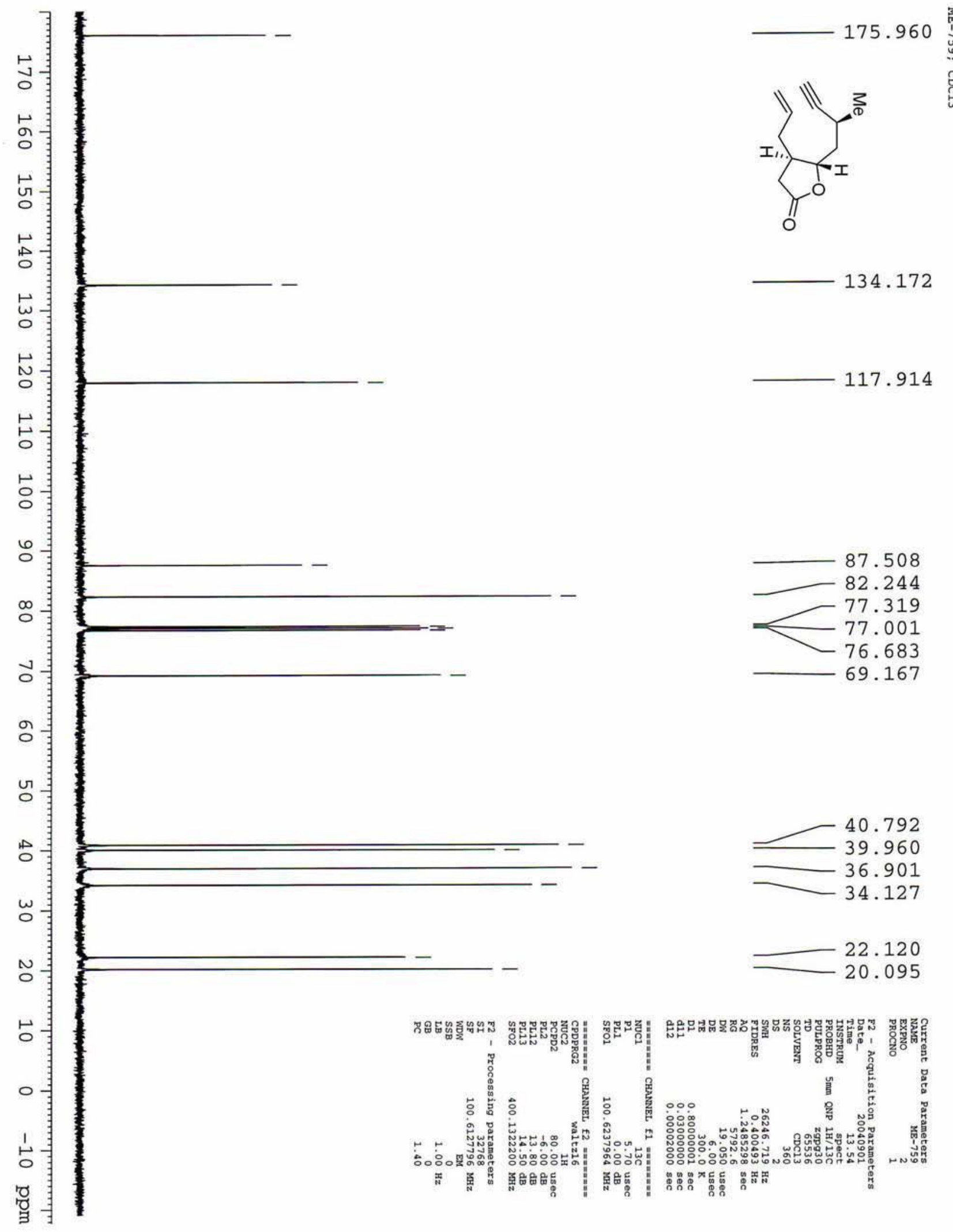

Page S 46 


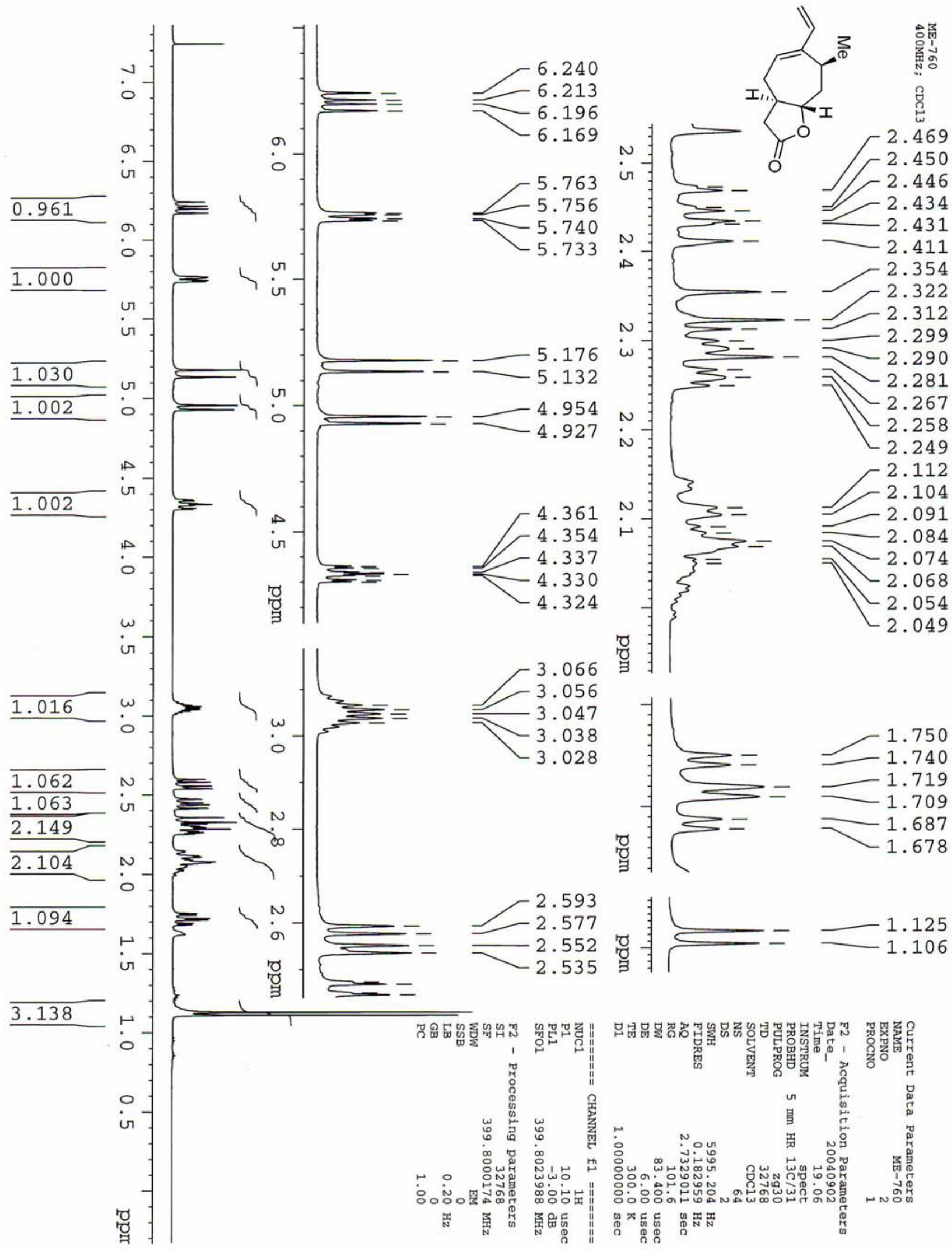




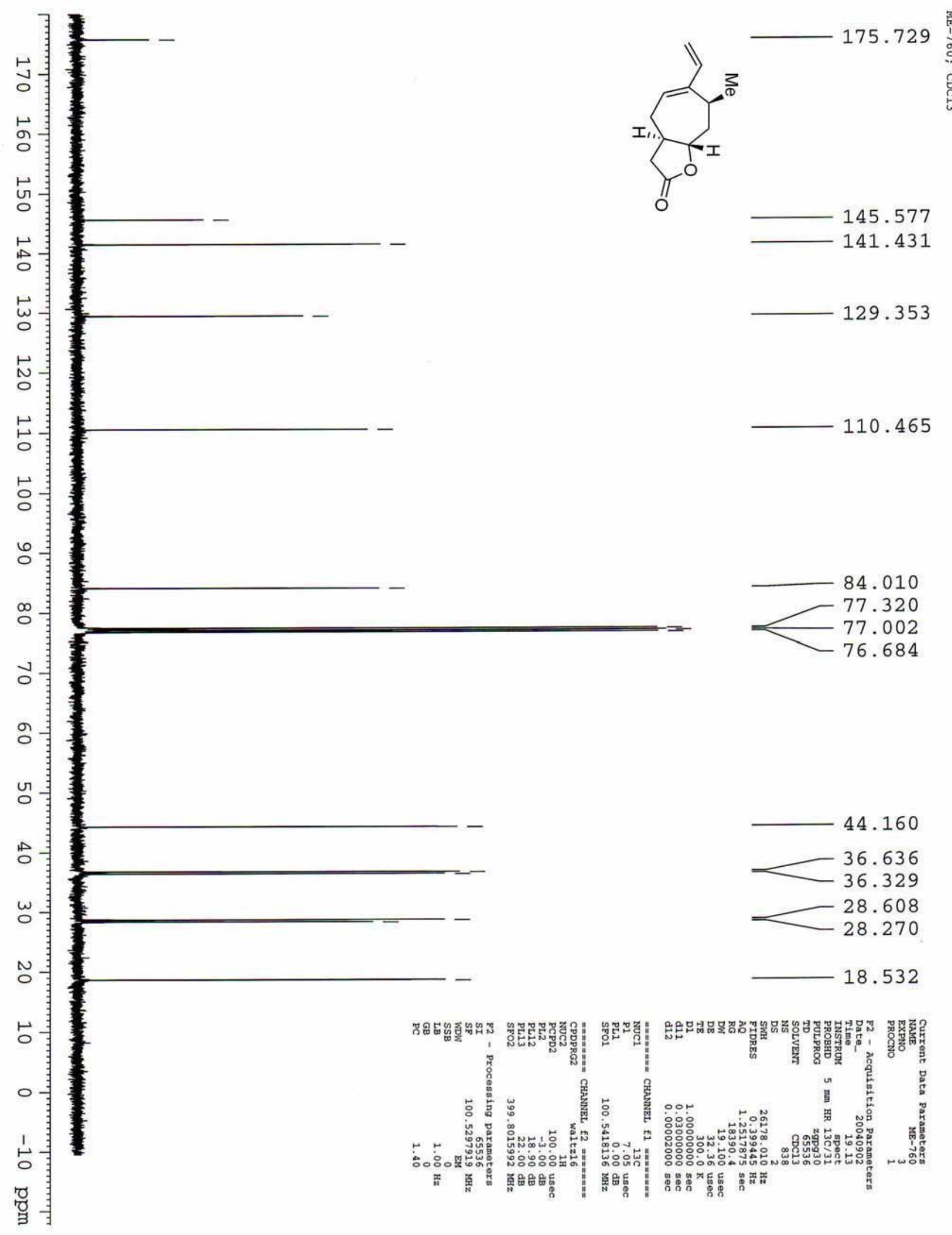

Page S 48 


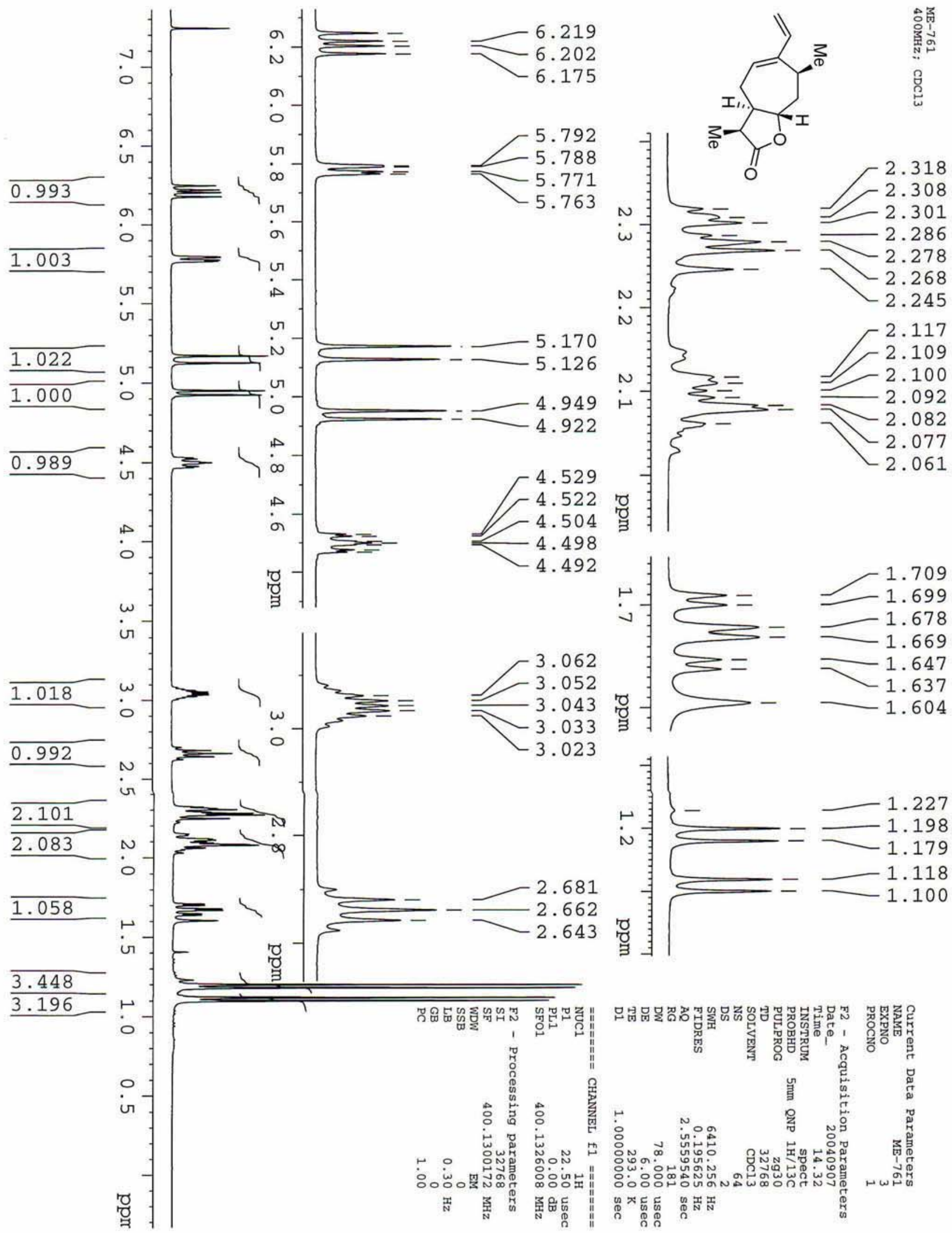



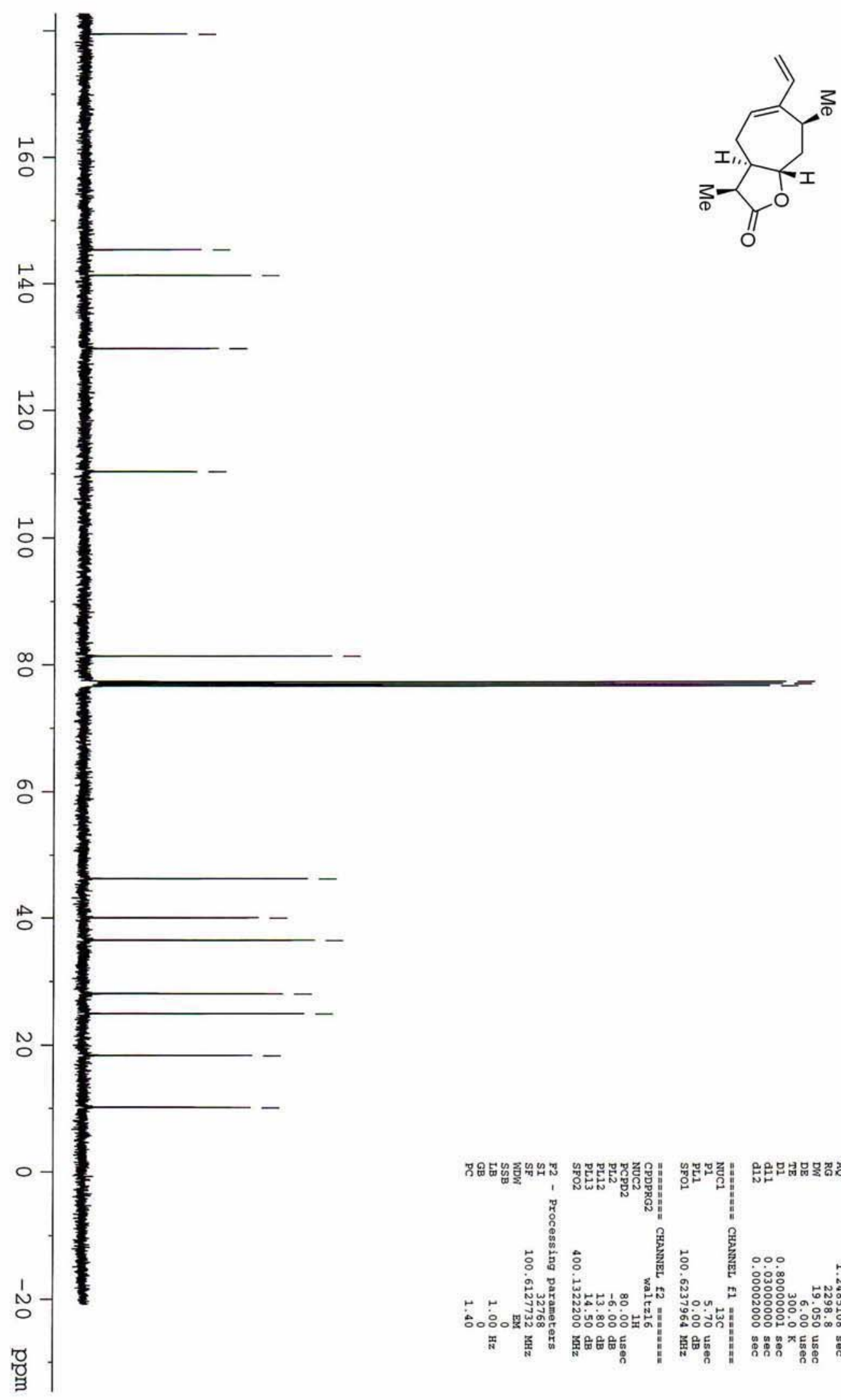

179.443

145.375

141.316

129.712

110.272
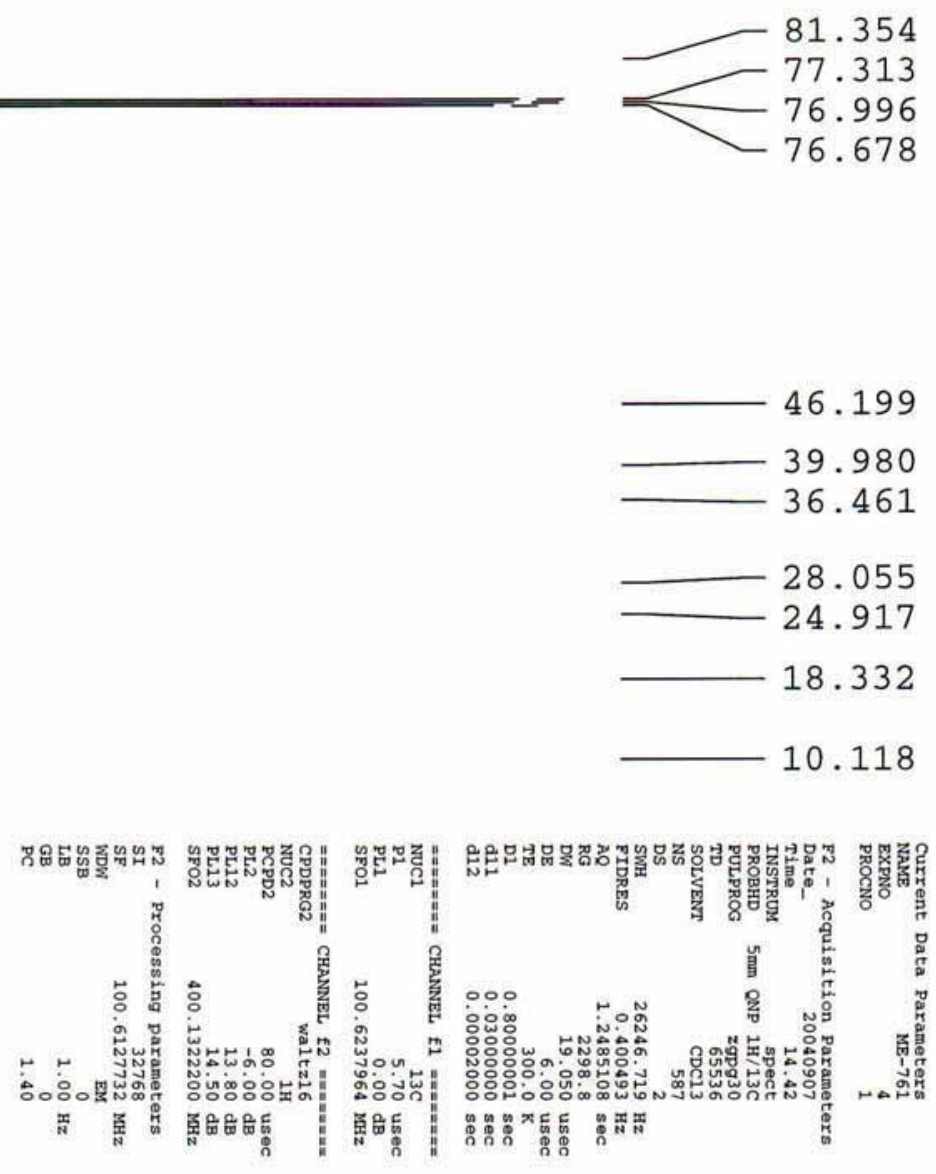

Page S 50 


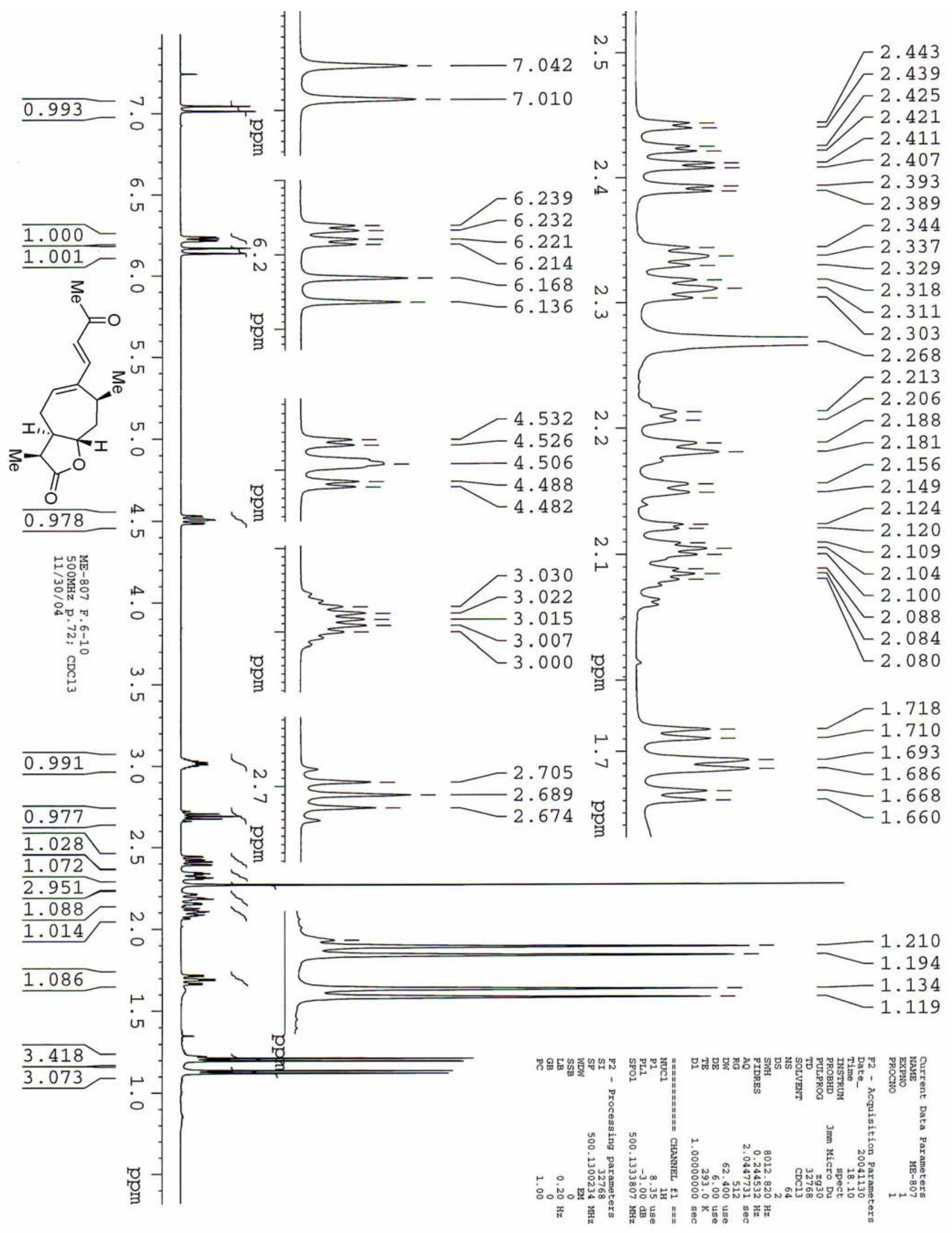



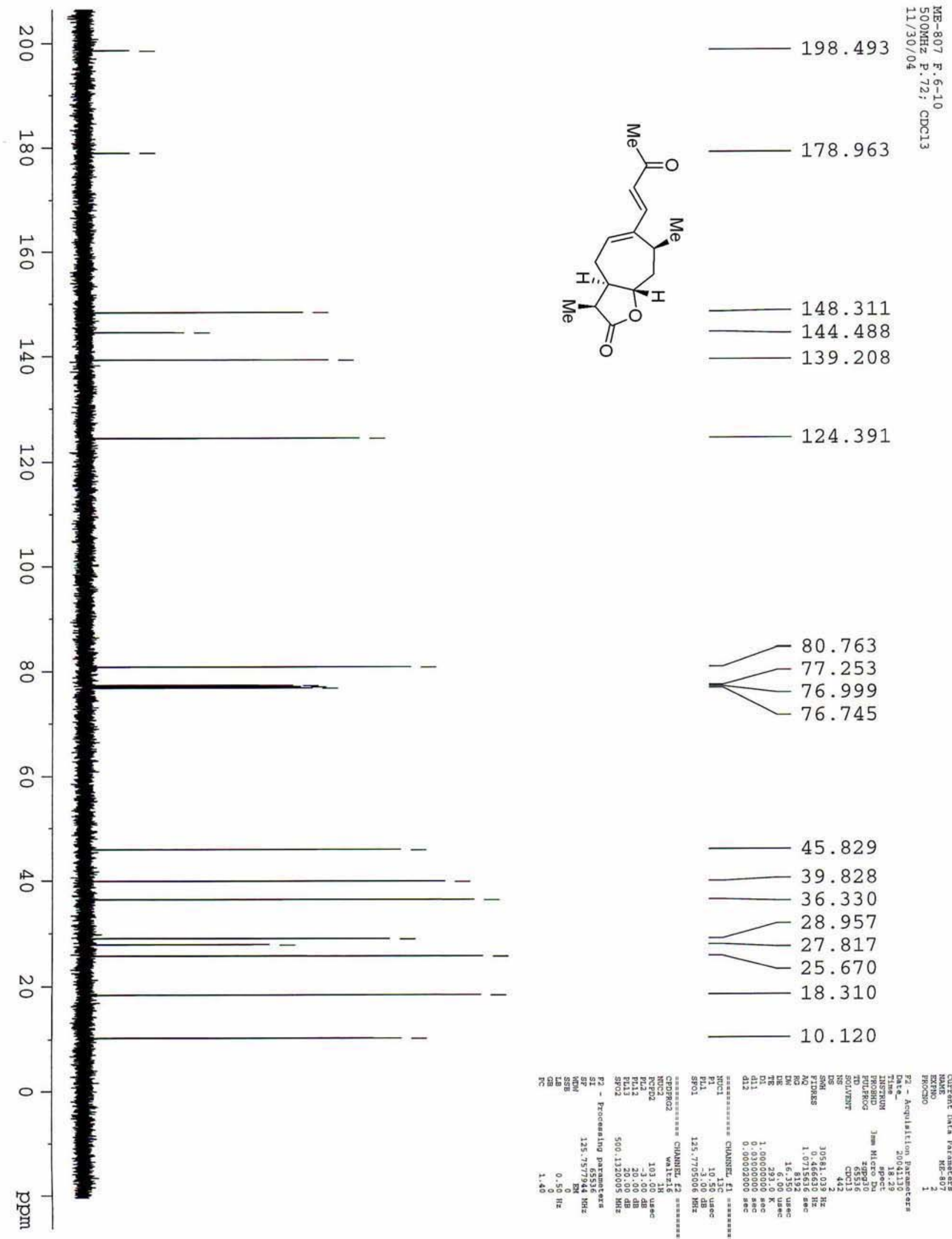

124.391
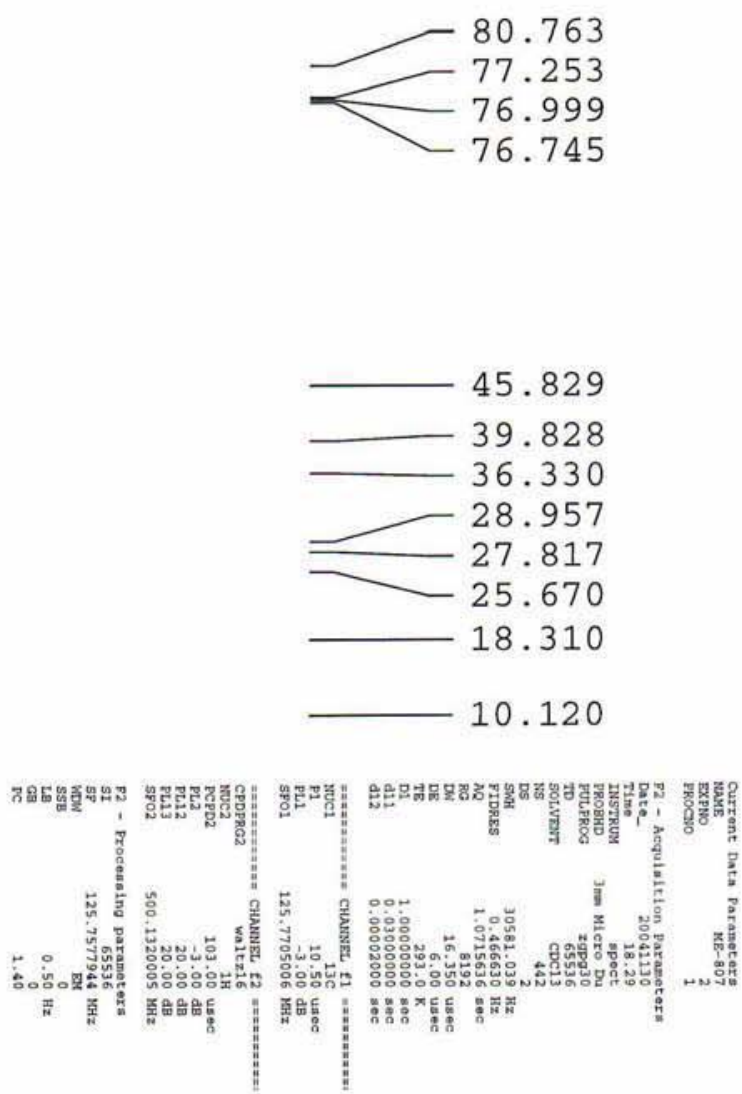

Page S 52 
Page S 53 